U.S. GEOLOGICAL SURVEY CIRCULAR 1044

\title{
Catalogue of U.S. Geological Survey Strong-Motion Records, 1987
}
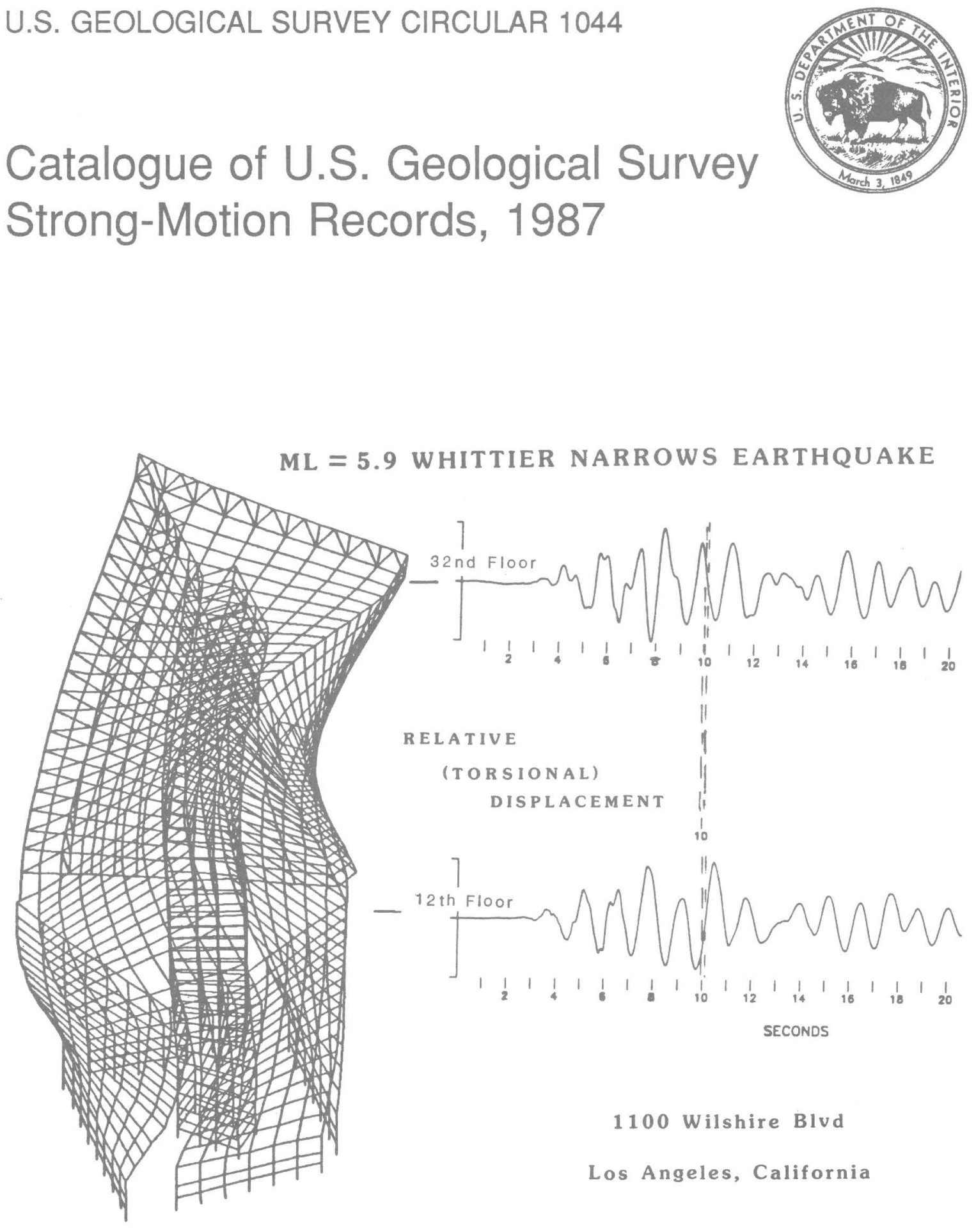


\section{AVAILABILITY OF BOOKS AND MAPS OF THE U.S. GEOLOGICAL SURVEY}

Instructions on ordering publications of the U.S. Geological Survey, along with prices of the last offerings, are given in the current-year issues of the monthly catalog "New Publications of the U.S. Geological Survey." Prices of available U.S. Geological Survey publications released prior to the current year are listed in the most recent annual "Price and Availability List." Publications that are listed in various U.S. Geological Survey catalogs (see back inside cover) but not listed in the most recent annual "Price and Availability List" are no longer available.

Prices of reports released to the open files are given in the listing "U.S. Geological Survey Open-File Reports," updated monthly, which is for sale in microfiche from the U.S. Geological Survey, Books and Open-File Reports Section, Federal Center, Box 25425, Denver, CO 80225. Reports released through the NTIS may be obtained by writing to the National Technical Information Service, U.S. Department of Commerce, Springfield, VA 22161; please include NTIS report number with inquiry.

Order U.S. Geological Survey publications by mail or over the counter from the offices given below.

\section{BY MAIL}

\section{Books}

Professional Papers, Bulletins, Water-Supply Papers, Techniques of Water-Resources Investigations, Circulars, publications of general interest (such as leaflets, pamphlets, booklets), single copies of Earthquakes \& Volcanoes, Preliminary Determination of Epicenters, and some miscellaneous reports, including some of the foregoing series that have gone out of print at the Superintendent of Documents, are obtainable by mail from

U.S. Geological Survey, Books and Open-File Reports Federal Center, Box 25425 Denver, CO 80225

Subscriptions to periodicals (Earthquakes \& Volcanoes and Preliminary Determination of Epicenters) can be obtained ONLY from the

\section{Superintendent of Documents \\ Government Printing Office \\ Washington, D.C. 20402}

(Check or money order must be payable to Superintendent of Documents.)

\section{Maps}

For maps, address mail orders to

$$
\begin{gathered}
\text { U.S. Geological Survey, Map Distribution } \\
\text { Federal Center, Box } 25286 \\
\text { Denver, CO } 80225
\end{gathered}
$$

Residents of Alaska may order maps from

$$
\begin{gathered}
\text { Alaska Distribution Section, U.S. Geological Survey, } \\
\text { New Federal Building - Box } 12 \\
101 \text { Twelfth Ave., Fairbanks, AK } 99701
\end{gathered}
$$

\section{OVER THE COUNTER}

\section{Books}

Books of the U.S. Geological Survey are available over the counter at the following Geological Survey Public Inquiries Offices, all of which are authorized agents of the Superintendent of Documents:

- WASHINGTON, D.C.--Main Interior Bldg., 2600 corridor, 18 th and C Sts., NW.

- DENVER, Colorado--Federal Bldg., Rm. 169, 1961 Stout St.

- LOS ANGELES, Callfornia--Federal Bldg., Rm. 7638, $300 \mathrm{~N}$. Los Angeles St.

- MENLO PARK, California--Bldg. 3 (Stop 533), Rm. 3128, 345 Middlefield Rd.

- RESTON, Virginia--503 National Center, Rm. 1C402, 12201 Sunrise Valley Dr.

- SALT LAKE CITY, Utah--Federal Bldg., Rm. 8105, 125 South State St.

- SAN FRANCISCO, California--Customhouse, Rm. 504, 555 Battery St.

- SPOKANE, Washington--U.S. Courthouse, Rm. 678, West 920 Riverside Ave..

- ANCHORAGE, Alaska--Rm. 101, 4230 University Dr.

- ANCHORAGE, Alaska--Federal Bldg, Rm. E-146, 701 C St.

\section{Maps}

Maps may be purchased over the counter at the U.S. Geological Survey offices where books are sold (all addresses in above list) and at the following Geological Survey offices:

- ROLLA, Missouri--1400 Independence Rd.

- DENVER, Colorado--Map Distribution, Bldg. 810, Federal Center

- FAIRBANKS, Alaska--New Federal Bldg., 101 Twelfth Ave. 
Catalogue of U.S. Geological Survey Strong-Motion Records, 1987

Compiled by RONALD L. PORCELLA and JOSEPHINE C. SWITZER

U.S. GEOLOGICAL SURVEY CIRCULAR 1044 


\title{
DEPARTMENT OF THE INTERIOR \\ MANUEL LUJAN, JR., Secretary
}

\author{
U.S. GEOLOGICAL SURVEY \\ Dallas L. Peck, Director
}

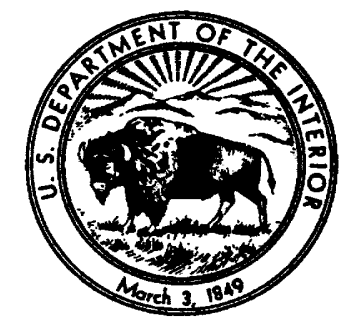

Any use of trade, product, or firm names in this publication is for descriptive purposes only and does not imply endorsement by the U.S. Government

UNITED STATES GOVERNMENT PRINTING OFFICE, WASHINGTON : 1989

Free on application to the

Books and Open-File Reports Section, U.S. Geological Survey, Federal Center, Box 25425, Denver, CO 80225

Library of Congress Catalog Card No. 83-600616

Front Cover: This figure is a finite element model showing the relative displacements (rotation time history) measured at the 12th and 32d floors of the JCG Finance Corp. building at 1100 Wilshire Boulevard in Los Angeles during the $M_{L}=5.9$ Whittier Narrows earthquake (Celebi and others, 1989). The 33-story building is a 21 -story triangular-in-plan tower above a 12-story rectangular-in-plan structure; a common rigid frame in the core connects the two configurations. The earthquake was recorded on 21 acceleration sensors strategically located at the basement, ground, 12th, 13th, and 32d floors so as to detect any translational, torsional, or rocking motions of the structure. These records show that the building responded in the coupled translation torsional mode; however, the torsional is a second mode such that the upper triangular tower rotated in an opposite sense when compared to the lower rectangular structure.

Reference: Celebi, M., Safak, E., and Youssef, N., 1989, Analysis of recorded responses of a unique building in Los Angeles to motions caused by the Whittier Narrows, California earthquake of October 1, 1987: U.S. Geological Survey Open-File Report 89-542, 77 p. 


\section{PREFACE}

The first seismic engineering program in the United States was administered by the Seismological Field Survey (SFS) of the Coast and Geodetic Survey. This program was begun in 1931 and essentially remained the responsibility of the SFS until 1973, when the U.S. Geological Survey (USGS) assimilated the program into its National Earthquake Hazards Reduction Program. The current Federal seismic engineering program operates the National Cooperative Strong-Motion Network (NCSMN) with nearly 1,000 stations in 41 States and Puerto Rico. This network is administered by the USGS in cooperation with both private industry and numerous Federal, State, and local agencies and organizations. Major contributors include the Army Corps of Engineers, the Veterans Administration, and the Metropolitan Water District of Southern California. Primary objectives of the program are to record strong ground motions and the response of representative engineered structures during moderate to large earthquakes, and to disseminate the resultant data and information about the records, sites, and structures to the earthquake engineering research and design community.

This catalogue continues in a revised format the yearly publication "StrongMotion Program Report, January-December [year]; it is a continuation of the table 1 summary of accelerograms recovered at NCSMN stations that had been published in that format since 1974. This report includes all accelerograms recovered during 1987. Unless otherwise noted, event data are from the "Preliminary Determination of Epicenters, " published weekly by the U.S. Geological Survey. 



\title{
CONTENTS
}

\author{
Preface III \\ Introduction 1 \\ References 1
}

TABLE

1. Catalogue of National Cooperative Strong-Motion Network accelerograph records recovered during 1987 


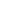




\title{
Catalogue of U.S. Geological Survey Strong-Motion Records, 1987
}

\author{
Compiled by Ronald L. Porcella and Josephine C. Switzer
}

\section{INTRODUCTION}

Nearly 550 accelerograph records were recovered from the National Cooperative Strong-Motion Network (NCSMN) during 1987. This compares with an annual average of 247 records for the period 1974 through 1986. U.S. Geological Survey NCSMN stations in California, Alaska, and Hawaii recorded 11 earthquakes of $M=5.0$ or greater during 1987 , including the $M_{L}=5.9$ Whittier Narrows earthquake on October 1 and the $M_{L}=6.1$ Superstition Hills earthquake on November 24 .

Accelerographs at 52 NCSMN stations in the Los Angeles region were triggered by the October 1 Whittier Narrows main shock and produced 358 channels of acceleration data. These data include recordings from extensively instrumented structures such as high-rise buildings in Los Angeles, Norwalk, and Newport Beach, a hospital in Loma Linda, a reservoir near Live Oak, and a base-isolated pipeline bridge across the Santa Ana River.

Maximum recorded acceleration was $0.63 \mathrm{~g}$ at the basement level of a 10-story building in Whittier. Within an epicentral distance of $25 \mathrm{~km}, 14$ stations produced peak horizontal ground motions in excess of $0.15 \mathrm{~g}$ (see Etheredge and Porcella, 1987).

The $\mathrm{M}_{\mathrm{L}}=5.3$ Whittier Narrows aftershock on October 4, 1987, produced strong-motion records at 30 of the 52 NCSMN main-shock stations. These stations include 11 buildings, 9 dams, 4 hospitals, and 5 ground sites at epicentral distances ranging from 2 to $57 \mathrm{~km}$. Significant ground motions were recorded at 6 stations within an epicentral distance of $12 \mathrm{~km}$ and are in the range 0.15 to $0.33 \mathrm{~g}$ (Etheredge and Porcella, 1988).

Two moderate-sized earthquakes occurred on November 24 in the northwest Imperial Valley of California. The first event $\left(M_{L}=5.8\right)$ triggered accelerographs at 24 NCSMN stations; the second event $\left(M_{L}=6.1\right)$ triggered instruments at 39 NCSMN stations. Six station records exceed $0.1 \mathrm{~g}$ for the first event, and 25 records from stations within $60 \mathrm{~km}$ contain peak horizontal ground motions in excess of $0.1 \mathrm{~g}$ (Porcella and others, 1987).

Additional magnitude 5 or greater earthquakes recorded at NCSMN stations in 1987, including the date, location, magnitude, and number of NCSMN stations triggered, are as follows: Feb. 4, Hawaii, 5.2, four records; Feb. 7, southern Calif., 5.4, one record; Feb. 14, central Calif., 5.2, two records; April 18, Alaska, 5.7, five records; July 31, northern Calif., 5.4, seven records; Nov. 11, Alaska, 7.0, four records; and Nov. 30, Alaska, 7.1, seven records. 


\section{REFERENCES}

Etheredge, E., and Porcella, R., 1987, Strong-motion data from the October 1, 1987 Whittier Narrows Earthquake: U.S. Geological Survey Open-File Report 87-616, 64 p.

Etheredge, E., and Porcella, R., 1988, Strong-motion data from the Whittier Narrows aftershock of October 4, 1987: U.S. Geological Survey Open-File Report 88-38, 32 p.

Porcella, R., Etheredge, E., Maley, R., and Switzer, J., 1987, Strong-motion data from the Superstition Hills earthquakes of 0154 and 1315 (Gmt), November 24, 1987: U.S. Geological Survey Open-File Report 87-672, 56 p. 
Table 1. Catalogue of National Cooperative Strong-Motion Network accelerograph records recovered during $19 \Omega 7$

[Station owners: ACOE, U.S. Army Corps of Engineers; BECH, Bechtel Power Corporation; CDOT, California Department of Transportation; CDWR, California Department of Water Resources; $\mathrm{CI}^{2}$, California Institute of Technology; DOE, U.S. Department of Energy; GLDN, U.S. Geological Survey, Golden, Colorado; JCG, JCG Finance Corporation of America; MANC, Municipality of Anchorage, Alaska; MWD, Los Angeles Metropolitan Water District; USGS, U.S. Geological Survey; VA, U.S. Veterans Administration. Instrument trigger time in minutes and seconds after the hour listed in earthquake column. Trigger time in brackets is P-wave arrival time as event occurred while instrument was in operation. S-minus trigger denotes $\underline{S}$-wave-arrival-minustrigger-time ( $\underline{S}-t)$ or $\underline{S}$-wave-minus-P-wave-arrival time ( $\underline{S}-\mathrm{P}$, in brackets) interval. Direction is of case acceleration for upward trace deflection on accelerogram; horizontal components are listed as azimuth, and vertical components as "up" or "down." Maximum amplitude is peak acceleration recorded at ground level on one vertical and two orthogonal horizontal components unless otherwise noted. Duration is interval between first and last peaks of acceleration greater than $0.10 \mathrm{~g}$.

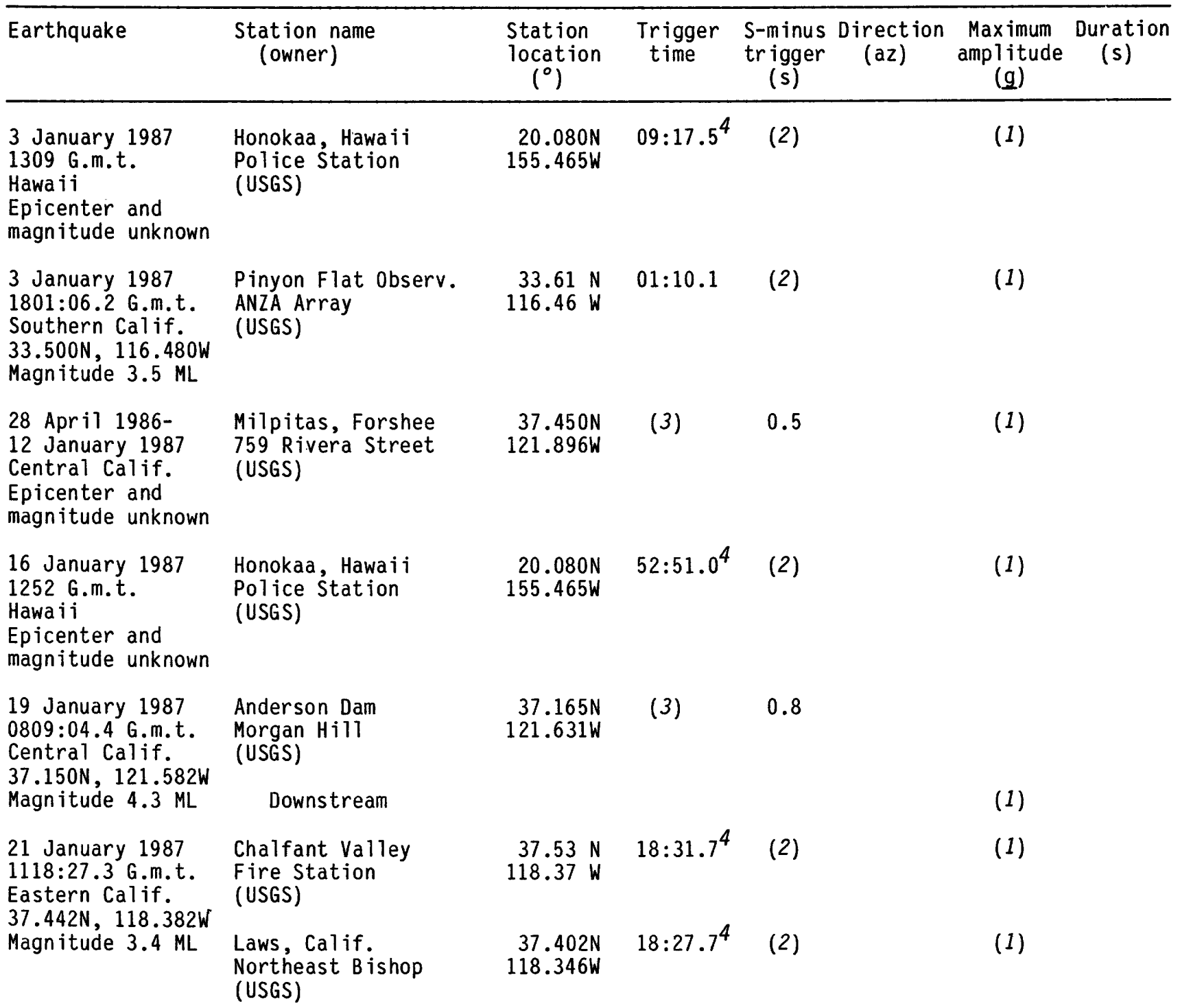


Table 1. Catalogue of National Cooperative Strong-Motion Network accelerograph records recovered during 1987-Continued

\begin{tabular}{|c|c|c|c|c|c|c|c|}
\hline Earthquake & $\begin{array}{l}\text { Station name } \\
\text { (owner) }\end{array}$ & $\begin{array}{l}\text { Station } \\
\text { location } \\
\left({ }^{\circ}\right)\end{array}$ & $\begin{array}{l}\text { Trigger } \\
\text { time }\end{array}$ & $\begin{array}{l}\text { S-minus } \\
\text { trigger } \\
(\mathrm{s})\end{array}$ & $\begin{array}{l}\text { Direction } \\
\quad(a z)\end{array}$ & $\begin{array}{l}\text { Maximum } \\
\text { amplitude } \\
(\mathrm{g})\end{array}$ & $\begin{array}{l}\text { Duration } \\
\text { (s) }\end{array}$ \\
\hline $\begin{array}{l}21 \text { January } 1987 \\
1142: 57.1 \text { G.m.t. } \\
\text { Eastern Calif. } \\
37.447 \mathrm{~N}, 118.382 \mathrm{~W} \\
\text { Magnitude } 3.5 \mathrm{ML}\end{array}$ & $\begin{array}{l}\text { Chalfant Valley } \\
\text { Fire Station } \\
\text { (USGS) }\end{array}$ & $\begin{array}{r}37.53 \mathrm{~N} \\
118.37 \mathrm{~W}\end{array}$ & $43: 01.4^{4}$ & 2.3 & & (1) & \\
\hline $\begin{array}{l}27 \text { January } 1987 \\
1757: 11.3 \text { G.m.t. } \\
\text { Eastern Calif. } \\
37.608 \mathrm{~N}, 118.463 \mathrm{~W} \\
\text { Magn itude } 3.8 \mathrm{ML}\end{array}$ & $\begin{array}{l}\text { South Hammil Valley } \\
\text { White Mountain Ranch } \\
\text { (USGS) }\end{array}$ & $\begin{array}{r}37.62 \mathrm{~N} \\
118.39 \mathrm{~W}\end{array}$ & $57: 16.6^{4}$ & 1.4 & & (1) & \\
\hline \multirow{4}{*}{$\begin{array}{l}4 \text { February } 1987 \\
0222: 32.7 \text { G.m.t. } \\
\text { Hawaii } \\
20.053 \mathrm{~N}, 156.530 \mathrm{~W} \\
\text { Magn itude } 5.2 \mathrm{ML}\end{array}$} & $\begin{array}{l}\text { Honokaa, Hawaii } \\
\text { Police Station } \\
\text { (USGS) }\end{array}$ & $\begin{array}{r}20.080 \mathrm{~N} \\
155.465 \mathrm{~W}\end{array}$ & $23: 04.1^{4}$ & (2) & & (1) & \\
\hline & $\begin{array}{l}\text { Kealakekua, Hawaii } \\
\text { Kona Hospital } \\
\text { (USGS) }\end{array}$ & $\begin{array}{r}19.523 \mathrm{~N} \\
155.879 \mathrm{~W}\end{array}$ & $22: 58.7^{4}$ & (2) & & (1) & \\
\hline & $\begin{array}{l}\text { Kapaau, Hawaii } \\
\text { Kohala Police Station } \\
\text { (USGS) }\end{array}$ & $\begin{array}{r}20.230 \mathrm{~N} \\
155.801 \mathrm{~W}\end{array}$ & $22: 57.4^{4}$ & (2) & $\begin{array}{l}102 \\
U p \\
012\end{array}$ & $\begin{array}{l}.06 \\
.03 \\
.04\end{array}$ & --- \\
\hline & $\begin{array}{l}\text { Mauna Kea, Hawaii } \\
\text { State Park } \\
\text { (USGS) }\end{array}$ & $\begin{array}{r}19.752 \mathrm{~N} \\
155.530 \mathrm{~W}\end{array}$ & $23: 04.2^{4}$ & (2) & & (1) & \\
\hline $\begin{array}{l}7 \text { February } 1987 \\
0345: 14.7 \text { G.m.t. } \\
\text { Southern Calif. } \\
32.390 \mathrm{~N}, 115.310 \mathrm{~W} \\
\text { Magn itude } 5.4 \mathrm{ML}\end{array}$ & $\begin{array}{l}\text { Calexico } \\
\text { Fire Station } \\
\text { (USGS) }\end{array}$ & $\begin{array}{r}32.67 \mathrm{~N} \\
115.49 \mathrm{~W}\end{array}$ & $45: 23.3$ & 4.3 & & (1) & \\
\hline \multirow{3}{*}{$\begin{array}{l}14 \text { February } 1987 \\
0726: 51.7 \text { G.m.t. } \\
\text { Central Calif. } \\
36.148 \mathrm{~N}, 120.335 \mathrm{~W} \\
\text { Magnitude } 5.2 \mathrm{ML}\end{array}$} & $\begin{array}{l}\text { Coalinga } \\
\text { 0il City } \\
\text { (USGS) }\end{array}$ & $\begin{array}{r}36.229 \mathrm{~N} \\
120.360 \mathrm{~W}\end{array}$ & $26: 54.3$ & 2.2 & $\begin{array}{l}360 \\
\text { Up } \\
270\end{array}$ & $\begin{array}{l}.21 \\
.07 \\
.26\end{array}$ & $\begin{array}{l}0.4 \\
--- \\
0.6\end{array}$ \\
\hline & $\begin{array}{l}\text { Hidden Dam } \\
\text { (ACOE) }\end{array}$ & $\begin{array}{r}37.112 \mathrm{~N} \\
119.883 \mathrm{~W}\end{array}$ & $27: 22.5$ & (2) & & & \\
\hline & Left Crest & & & & & (1) & \\
\hline $\begin{array}{l}21 \text { February } 1987 \\
1205: 32.4 \text { G.m.t. } \\
\text { Eastern Calif. } \\
37.510 \mathrm{~N}, 118.400 \mathrm{~W} \\
\text { Magnitude } 3.1 \mathrm{ML}\end{array}$ & $\begin{array}{l}\text { Chalfant Valley } \\
\text { Fire Station } \\
\text { (USGS) }\end{array}$ & $\begin{array}{r}37.53 \mathrm{~N} \\
118.37 \mathrm{~W}\end{array}$ & $05: 36.8^{4}$ & 0.7 & & (1) & \\
\hline $\begin{array}{l}21 \text { February } 1987 \\
1302: 19.6 \text { G.m.t. } \\
\text { Central Calif. } \\
36.590 \mathrm{~N}, 121.228 \mathrm{~W} \\
\text { Magnitude } 3.3 \mathrm{ML}\end{array}$ & $\begin{array}{l}\text { Bear Valley Station } 1 \\
\text { CDF Fire Station } \\
\text { (USGS) }\end{array}$ & $\begin{array}{r}36.573 \mathrm{~N} \\
121.184 \mathrm{~W}\end{array}$ & $02: 27.1$ & (2) & & (1) & \\
\hline
\end{tabular}


Table 1. Catalogue of National Cooperative Strong-Motion Network accelerograph records recovered during 1987-Continued

\begin{tabular}{|c|c|c|c|c|c|c|c|}
\hline Earthquake & $\begin{array}{l}\text { Station name } \\
\text { (owner) }\end{array}$ & $\begin{array}{l}\text { Station } \\
\text { location } \\
\left({ }^{\circ}\right)\end{array}$ & $\begin{array}{l}\text { Trigger } \\
\text { time }\end{array}$ & $\begin{array}{l}\text { S-minus } \\
\text { trigger } \\
(\mathrm{s})\end{array}$ & $\begin{array}{c}\text { Direction } \\
(\mathrm{az})\end{array}$ & $\begin{array}{l}\text { Maximum } \\
\text { amplitude } \\
(\mathrm{g})\end{array}$ & $\begin{array}{l}\text { Duration } \\
\text { (s) }\end{array}$ \\
\hline $\begin{array}{l}21 \text { February } 1987 \\
2315: 29.9 \text { G.m.t. } \\
\text { Southern Calif. } \\
34.130 \mathrm{~N}, 117.450 \mathrm{~W} \\
\text { Magnitude } 3.9 \mathrm{ML}\end{array}$ & $\begin{array}{l}\text { Sycamore Forest Sta. } \\
\text { Angeles Forest } \\
\text { (USGS) }\end{array}$ & $\begin{array}{r}34.193 \mathrm{~N} \\
117.426 \mathrm{~W}\end{array}$ & $15: 33.7$ & (2) & & (1) & \\
\hline \multirow{6}{*}{$\begin{array}{l}25 \text { February } 1987 \\
0615 \text { G.m.t. } \\
\text { Eastern Calif. } \\
\text { Epicenter and } \\
\text { magnitude unknown }\end{array}$} & $\begin{array}{l}\text { McGee Creek } \\
\text { Mammoth Lakes } \\
\text { (USGS) SMA-1 }\end{array}$ & $\begin{array}{r}37.550 \mathrm{~N} \\
118.811 \mathrm{~W}\end{array}$ & $15: 44.2$ & (2) & & (1) & \\
\hline & $\begin{array}{l}\text { McGee Creek } \\
\text { Mammoth Lakes } \\
\text { (USGS) CRA-1 }\end{array}$ & $\begin{array}{r}37.550 \mathrm{~N} \\
118.811 \mathrm{~W}\end{array}$ & $15: 44.2$ & (2) & & & \\
\hline & 166 m downhole & & & & & (1) & \\
\hline & $35 \mathrm{~m}$ downhole & & & & & (1) & \\
\hline & Surface & & & & & (1) & \\
\hline & $1 \mathrm{~m}$ downhole & & & & & (1) & \\
\hline \multirow{2}{*}{$\begin{array}{l}26 \text { February } 1987 \\
1302: 00.2 \text { G.m.t. } \\
\text { Eastern Cal if. } \\
37.547 \mathrm{~N}, 118.890 \mathrm{~W} \\
\text { Magn itude } 3.5 \mathrm{ML}\end{array}$} & $\begin{array}{l}\text { McGee Creek } \\
\text { Mammoth Lakes } \\
\text { (USGS) SMA-1 }\end{array}$ & $\begin{array}{r}37.550 \mathrm{~N} \\
118.811 \mathrm{~W}\end{array}$ & $02: 00.5$ & 0.8 & $\begin{array}{l}360 \\
\text { Up } \\
270\end{array}$ & $\begin{array}{l}.09 \\
.04 \\
.05\end{array}$ & $\begin{array}{l}--- \\
-\cdots \\
---\end{array}$ \\
\hline & Note: CRA-1 failed & during 26 & February e & thquake & e $(1302: 00$ & 2 G.m.t.). & \\
\hline \multirow{6}{*}{$\begin{array}{l}27 \text { February } 1987 \\
1624: 57.7 \text { G.m.t. } \\
\text { Eastern Cal if. } \\
37.542 \mathrm{~N}, 118.887 \mathrm{~W} \\
\text { Magn itude } 3.9 \mathrm{ML}\end{array}$} & $\begin{array}{l}\text { McGee Creek } \\
\text { Mammoth Lakes } \\
\text { (USGS) SMA-1 }\end{array}$ & $\begin{array}{r}37.550 \mathrm{~N} \\
118.811 \mathrm{~W}\end{array}$ & $24: 58.9$ & (2) & $\begin{array}{l}360 \\
\text { Up } \\
270\end{array}$ & $\begin{array}{l}.06 \\
.03 \\
.05\end{array}$ & $\begin{array}{l}--- \\
---\end{array}$ \\
\hline & $\begin{array}{l}\text { McGee Creek } \\
\text { Mammoth Lakes } \\
\text { (USGS) CRA-1 }\end{array}$ & $\begin{array}{r}37.550 \mathrm{~N} \\
118.811 \mathrm{~W}\end{array}$ & $24: 58.9$ & (2) & & & \\
\hline & $166 \mathrm{~m}$ downhole & & & & & (1) & \\
\hline & 35 m downhole & & & & & (1) & \\
\hline & Surface & & & & $\begin{array}{l}360 \\
\text { Up } \\
270\end{array}$ & $\begin{array}{l}.06 \\
.05 \\
.06\end{array}$ & --- \\
\hline & $1 \mathrm{~m}$ downhole & & & & & (1) & \\
\hline $\begin{array}{l}1 \text { March } 1987 \\
0911: 50.3 \mathrm{G} . \mathrm{m.t} . \\
\text { Eastern Calif. } \\
37.482 \mathrm{~N}, 118.853 \mathrm{~W} \\
\text { Magnitude } 3.1 \mathrm{ML}\end{array}$ & $\begin{array}{l}\text { McGee Creek } \\
\text { Mammoth Lakes } \\
\text { (USGS) SMA-1 }\end{array}$ & $\begin{array}{r}37.550 \mathrm{~N} \\
118.811 \mathrm{~W}\end{array}$ & $11: 52.0$ & (2) & & (1) & \\
\hline
\end{tabular}


Table 1. Catalogue of National Cooperative Strong-Motion Network accelerograph records recovered during 1987-Continued

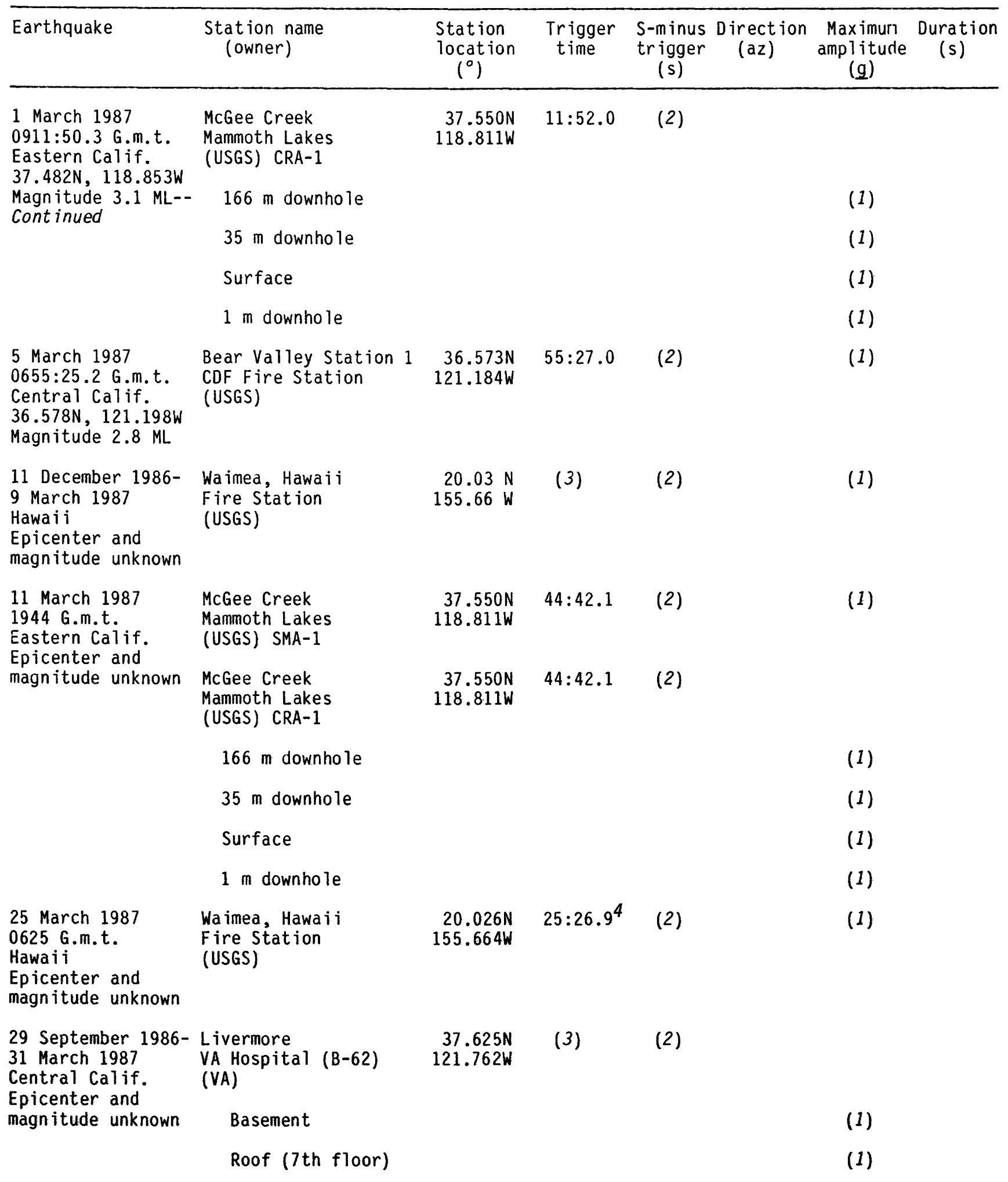


Table 1. Catalogue of National Cooperative Strong-Motion Network accelerograph records recovered during 1987-Continued

\begin{tabular}{|c|c|c|c|c|c|c|c|}
\hline Earthquake & $\begin{array}{l}\text { Station name } \\
\text { (owner) }\end{array}$ & $\begin{array}{l}\text { Station } \\
\text { location } \\
\left({ }^{\circ}\right)\end{array}$ & $\begin{array}{l}\text { Trigger } \\
\text { time }\end{array}$ & $\begin{array}{c}\text { S-minus } \\
\text { trigger } \\
(\mathrm{s})\end{array}$ & $\begin{array}{l}\text { Direction } \\
\quad(a z)\end{array}$ & $\begin{array}{l}\text { Maximum } \\
\text { amplitude } \\
(\mathrm{g})\end{array}$ & $\begin{array}{l}\text { Duration } \\
\text { (s) }\end{array}$ \\
\hline \multirow{3}{*}{$\begin{array}{l}10 \text { Apri1 } 1987 \\
0451: 46.4 \text { G.m.t. } \\
\text { Central Calif. } \\
37.563 \mathrm{~N}, 121.677 \mathrm{~W} \\
\text { Magn itude } 3.6 \mathrm{ML}\end{array}$} & $\begin{array}{l}\text { Livermore } \\
\text { VA Hospital (B-62) } \\
\text { (VA) }\end{array}$ & $\begin{array}{r}37.625 \mathrm{~N} \\
121.762 \mathrm{~W}\end{array}$ & (3) & 2.0 & & & \\
\hline & Basement & & & & & (1) & \\
\hline & Roof (7th floor) & & & & $\begin{array}{l}125 \\
\text { Up } \\
035\end{array}$ & $\begin{array}{l}.05 \\
.01 \\
.02\end{array}$ & $\begin{array}{l}--- \\
--- \\
---\end{array}$ \\
\hline $\begin{array}{l}16 \text { July 1986- } \\
11 \text { April } 1987\end{array}$ & $\begin{array}{l}\text { ANZA Array } \\
\text { Rarick Springs }\end{array}$ & $\begin{array}{r}33.57 \mathrm{~N} \\
116.52 \mathrm{~W}\end{array}$ & (3) & (2) & & (1) & \\
\hline
\end{tabular}

Southern Calif. (USGS)

Epicenters and

magnitudes unknown

Note: One additional record $(1)$ recovered at Rarick Springs.

$\begin{array}{llrrr}13 \text { April } 1987 & \text { Hollister } & 36.807 \mathrm{~N} & \text { (3) } & 1.7 \\ \text { 0947:00.9 G.m.t. } & \text { Damler Residence } & 121.408 \mathrm{~W} & & \\ \text { Central Calif. } & \text { (USGS) } & & \end{array}$

(1)

$36.853 \mathrm{~N}, 121.435 \mathrm{~W}$

Magnitude $2.7 \mathrm{ML}$

18 April 1987

$0201: 38.8$ G.m.t.

Southern Alaska

$61.374 \mathrm{~N}, 150.656 \mathrm{~W}$

Magnitude 5.7 MB

Anchorage 61.189N

Pacific University $149.801 \mathrm{~W}$

(3)

(2)

360
Up
270

.05

(USGS)

Anchorage $\quad 61.230 \mathrm{~N}$

(3)

8.6

(1)

(MANC)

Anchorage

$149.864 \mathrm{~W}$

8.6

.05

$---$

Fire Station No. $4 \quad 149.848 \mathrm{~W}$

(3)

(2)

(1)

(MANC)

Note: 2 additional records ${ }^{1}$ recovered at Fire Station No. 4.

$\begin{array}{llll}\text { Anchorage } & 61.21 \mathrm{~N} & 01: 50.3^{4} & 8.8\end{array}$

Fire Station No. $6 \quad 149.75 \mathrm{~W}$

(1)

(USGS/GLDN)

Anchorage

Fire Station No. $7 \quad 149.950 \mathrm{~W}$

(3)

9.0

(1)

(MANC)

12 October 1986-

Sunol Wilderness

$37.515 \mathrm{~N}$

(3)

0.4

(1)

26 April 1987

Central Calif.

Regional Park

$121.830 \mathrm{~W}$

(USGS)

magnitude unknown

27 Apri1 1987

Honokaa, Hawa i

Police Station

$20.080 \mathrm{~N}$

$41: 13.1^{4}$

(2)

(1)

1641 G.m.t.

(USGS)

$155.465 \mathrm{~W}$

Epicenter and

magnitude unknown 
Table 1. Catalogue of National Cooperative Strong-Motion Network accelerograph records recovered during 1987-Continued

\begin{tabular}{|c|c|c|c|c|c|c|c|}
\hline Earthquake & $\begin{array}{l}\text { Station name } \\
\text { (owner) }\end{array}$ & $\begin{array}{l}\text { Station } \\
\text { location } \\
\left({ }^{\circ}\right)\end{array}$ & $\begin{array}{l}\text { Trigger } \\
\text { time }\end{array}$ & $\begin{array}{l}\text { S-minus } \\
\text { trigger } \\
(\mathrm{s})\end{array}$ & $\begin{array}{l}\text { Direction } \\
\quad(a z)\end{array}$ & $\begin{array}{l}\text { Maximum } \\
\text { amplitude } \\
(\mathrm{g})\end{array}$ & $\begin{array}{l}\text { Duration } \\
\text { (s) }\end{array}$ \\
\hline $\begin{array}{l}27 \text { Apri1 } 1987 \\
1741: 24.0 \mathrm{G.m.t} \text {. } \\
\text { Southern Calif. } \\
33.060 \mathrm{~N}, 115.570 \mathrm{~W} \\
\text { Magnitude } 3.5 \mathrm{ML}\end{array}$ & $\begin{array}{l}\text { Imperial Wild life } \\
\text { Liquefaction Array } \\
\text { (USGS) }\end{array}$ & $\begin{array}{r}33.097 \mathrm{~N} \\
115.530 \mathrm{~W}\end{array}$ & (3) & 1.6 & & (1) & \\
\hline $\begin{array}{l}30 \text { April } 1987 \\
1924: 22.2 \text { G.m.t. } \\
\text { Central Calif. } \\
36.840 \mathrm{~N}, 121.283 \mathrm{~W} \\
\text { Magnitude } 4.1 \mathrm{ML}\end{array}$ & $\begin{array}{l}\text { Hollister } \\
\text { Differential Array } \\
\text { (USGS) }\end{array}$ & $\begin{array}{r}36.888 \mathrm{~N} \\
121.413 \mathrm{~W}\end{array}$ & $24: 25.5$ & 2.6 & & (1) & \\
\hline $\begin{array}{l}15 \text { December 1986- } \\
6 \text { June } 1987 \\
\text { Central Calif. } \\
\text { Epicenters and } \\
\text { magnitudes unknown }\end{array}$ & $\begin{array}{l}\text { Bear Valley Station } 10 \\
\text { Webb Residence } \\
\text { (USGS) } \\
\text { Note: Five additiona }\end{array}$ & $\begin{array}{l}36.532 \mathrm{~N} \\
121.143 \mathrm{~W} \\
\text { a] records } 1\end{array}$ & recovered & at Webb & $\begin{array}{l}310 \\
\text { Up } \\
220 \\
\text { Residence. }\end{array}$ & $\begin{array}{l}.06 \\
.04 \\
.05\end{array}$ & $\begin{array}{l}--- \\
--- \\
--\end{array}$ \\
\hline $\begin{array}{l}6 \text { June } 1987 \\
1623 \mathrm{G.m.t.} \\
\text { Centrai Calif. } \\
\text { Epicenter and } \\
\text { magnitude unknown }\end{array}$ & $\begin{array}{l}\text { Bear Valley Station } 10 \\
\text { Webb Res idence } \\
\text { (USGS) }\end{array}$ & $\begin{array}{r}36.532 \mathrm{~N} \\
121.143 \mathrm{~W}\end{array}$ & $23: 48.4$ & 1.1 & & (1) & \\
\hline $\begin{array}{l}21 \text { June } 1987 \\
0613 \text { G.m.t. } \\
\text { Hawaii } \\
\text { Epicenter and } \\
\text { magnitude unknown }\end{array}$ & $\begin{array}{l}\text { Waimea, Hawai i } \\
\text { Fire Station } \\
\text { (USGS) }\end{array}$ & $\begin{array}{r}20.026 \mathrm{~N} \\
155.664 \mathrm{~W}\end{array}$ & $13: 42.8^{4}$ & (2) & & (1) & \\
\hline \multirow{6}{*}{$\begin{array}{l}21 \text { June } 1987 \\
0616: 27.3 \mathrm{G.m.t} . \\
\text { Eastern Calif. } \\
37.550 \mathrm{~N}, 118.793 \mathrm{~W} \\
\text { Magnitude } 3.4 \mathrm{ML}\end{array}$} & $\begin{array}{l}\text { McGee Creek } \\
\text { Mammoth Lakes } \\
\text { (USGS) SMA-1 }\end{array}$ & $\begin{array}{r}37.550 \mathrm{~N} \\
118.811 \mathrm{~W}\end{array}$ & $16: 27.6$ & (2) & & (1) & \\
\hline & $\begin{array}{l}\text { McGee Creek } \\
\text { Mammoth Lakes } \\
\text { (USGS) CRA-1 }\end{array}$ & $\begin{array}{r}37.550 \mathrm{~N} \\
118.811 \mathrm{~W}\end{array}$ & $16: 27.6$ & (2) & & & \\
\hline & $166 \mathrm{~m}$ downhole & & & & & (1) & \\
\hline & $35 \mathrm{~m}$ downhole & & & & & (1) & \\
\hline & Surface & & & & & (1) & \\
\hline & $1 \mathrm{~m}$ downhole & & & & & (1) & \\
\hline \multirow{2}{*}{$\begin{array}{l}23 \text { October 1986- } \\
10 \text { July } 1987 \\
\text { Southern Calif. } \\
\text { Epicenters and } \\
\text { magnitudes unknown }\end{array}$} & $\begin{array}{l}\text { Whitewater Trout Farm } \\
\text { (USGS) }\end{array}$ & $\begin{array}{r}33.989 \mathrm{~N} \\
116.655 \mathrm{~W}\end{array}$ & (3) & (2) & & (1) & \\
\hline & Note: Three addition & nat records ${ }^{1}$ & 1 recove & ed at $W r$ & tewater Tro & ut Farm. & \\
\hline
\end{tabular}


Table 1. Catalogue of National Cooperative Strong-Motion Network accelerograph records recovered during 1987-Continued

\begin{tabular}{|c|c|c|c|c|c|c|c|}
\hline Earthquake & $\begin{array}{l}\text { Station name } \\
\text { (owner) }\end{array}$ & $\begin{array}{l}\text { Station } \\
\text { location } \\
\left({ }^{\circ}\right)\end{array}$ & $\begin{array}{l}\text { Trigger } \\
\text { time }\end{array}$ & $\begin{array}{l}\text { S-minus } \\
\text { trigger } \\
(\mathrm{s})\end{array}$ & $\begin{array}{l}\text { Direction } \\
\quad(a z)\end{array}$ & $\begin{array}{l}\text { Maximum } \\
\text { amplitude } \\
(\mathrm{g})\end{array}$ & $\begin{array}{l}\text { Duration } \\
\text { (s) }\end{array}$ \\
\hline \multirow{2}{*}{$\begin{array}{l}12 \text { July } 1987 \\
1937: 30.9 \text { G.m.t. } \\
\text { Eastern Calif. } \\
37.520 \mathrm{~N}, 118.470 \mathrm{~W} \\
\text { Magnitude } 3.1 \mathrm{ML}\end{array}$} & $\begin{array}{l}\text { Chalfant Valley } \\
\text { Fire Station } \\
\text { (USGS) }\end{array}$ & $\begin{array}{r}37.53 \mathrm{~N} \\
118.37 \mathrm{~W}\end{array}$ & $37: 40.1^{4}$ & (2) & $\begin{array}{l}360 \\
\text { Up } \\
270\end{array}$ & $\begin{array}{l}.05 \\
.03 \\
.04\end{array}$ & $\begin{array}{l}-- \\
--- \\
--\end{array}$ \\
\hline & $\begin{array}{l}\text { Laws, Cal if. } \\
\text { Northeast B ishop } \\
\text { (USGS) }\end{array}$ & $\begin{array}{r}37.402 \mathrm{~N} \\
118.346 \mathrm{~W}\end{array}$ & $37: 30.7^{4}$ & (2) & & (1) & \\
\hline $\begin{array}{l}29 \text { July } 1987 \\
0352: 32.5 \mathrm{G} . \mathrm{m} . \mathrm{t} . \\
\text { Eastern Calif. } \\
38.367 \mathrm{~N}, 118.170 \mathrm{~W} \\
\text { Magnitude } 4.6 \mathrm{ML}\end{array}$ & $\begin{array}{l}\text { Mina, Nevada } \\
\text { B lack Star Mine } \\
\text { (USGS) }\end{array}$ & $\begin{array}{r}38.434 \mathrm{~N} \\
118.154 \mathrm{~W}\end{array}$ & (3) & (2) & & (1) & \\
\hline \multirow{7}{*}{$\begin{array}{l}31 \text { July } 1987 \\
2356: 58.0 \mathrm{G} . \mathrm{m} . \mathrm{t} \text {. } \\
\text { Northern Cal if. } \\
40.415 \mathrm{~N}, 124.407 \mathrm{~W} \\
\text { Magnitude } 5.5 \mathrm{ML}\end{array}$} & $\begin{array}{l}\text { Eel River Valley Array } \\
\text { Bunker Hill FAA } \\
\text { (USGS) }\end{array}$ & $\begin{array}{r}40.498 \mathrm{~N} \\
124.294 \mathrm{~W}\end{array}$ & $57: 04.2$ & 0.7 & $\begin{array}{l}360 \\
\text { Up } \\
270\end{array}$ & $\begin{array}{l}.13 \\
.03 \\
.09\end{array}$ & $\begin{array}{l}--- \\
--- \\
---\end{array}$ \\
\hline & $\begin{array}{l}\text { Eel River Valley Array } \\
\text { Butler Valley Sta. } 2 \\
\text { (USGS) }\end{array}$ & $\begin{array}{r}40.79 \mathrm{~N} \\
123.88 \mathrm{~W}\end{array}$ & (3) & (2) & $\begin{array}{l}060 \\
\text { Up } \\
330\end{array}$ & $\begin{array}{l}.09 \\
.02 \\
.07\end{array}$ & $\begin{array}{l}--- \\
--- \\
--\end{array}$ \\
\hline & $\begin{array}{l}\text { Eel River Valley Array } \\
\text { Centerville Beach } \\
\text { (USGS) }\end{array}$ & $\begin{array}{r}40.563 \mathrm{~N} \\
124.348 \mathrm{~W}\end{array}$ & (3) & 2.6 & $\begin{array}{l}360 \\
\text { Up } \\
270\end{array}$ & $\begin{array}{l}.26 \\
.15 \\
.24\end{array}$ & $\begin{array}{l}3.5 \\
0.3 \\
2.7\end{array}$ \\
\hline & $\begin{array}{l}\text { Eel River Valley Array } \\
\text { College of Redwoods } \\
\text { (USGS) }\end{array}$ & $\begin{array}{r}40.699 \mathrm{~N} \\
124.200 \mathrm{~W}\end{array}$ & (3) & 6.0 & $\begin{array}{l}360 \\
\text { Up } \\
270\end{array}$ & $\begin{array}{l}.08 \\
.04 \\
.08\end{array}$ & $\begin{array}{l}--- \\
--- \\
--\end{array}$ \\
\hline & $\begin{array}{l}\text { Eel River Valley Array } \\
\text { Fortuna Fire Station } \\
\text { (USGS) }\end{array}$ & $\begin{array}{r}40.599 \mathrm{~N} \\
124.154 \mathrm{~W}\end{array}$ & (3) & 5.4 & $\begin{array}{l}360 \\
\text { Up } \\
270\end{array}$ & $\begin{array}{l}.22 \\
.07 \\
.08\end{array}$ & $\begin{array}{l}1.5 \\
-.- \\
---\end{array}$ \\
\hline & $\begin{array}{l}\text { Eel River Valley Array } \\
\text { Loleta Fire Station } \\
\text { (USGS) }\end{array}$ & $\begin{array}{r}40.644 \mathrm{~N} \\
124.219 \mathrm{~W}\end{array}$ & (3) & 5.5 & $\begin{array}{l}360 \\
\text { Up } \\
270\end{array}$ & $\begin{array}{l}.17 \\
.14 \\
.13\end{array}$ & $\begin{array}{l}2 \text { peaks } \\
0.9 \\
0.6\end{array}$ \\
\hline & $\begin{array}{l}\text { Eel River Valley Array } \\
\text { South Bay Union School } \\
\text { (USGS) }\end{array}$ & $\begin{array}{r}40.735 \mathrm{~N} \\
124.207 \mathrm{~W}\end{array}$ & (3) & 5.3 & $\begin{array}{l}360 \\
\text { Up } \\
270\end{array}$ & $\begin{array}{l}.05 \\
.04 \\
.11\end{array}$ & 1 peak \\
\hline $\begin{array}{l}3 \text { August } 1987 \\
0948: 03.7 \mathrm{G.m} . \mathrm{t} \text {. } \\
\text { Eastern Cal i.f. } \\
38.390 \mathrm{~N}, 118.060 \mathrm{~W} \\
\text { Magnitude } 4.4 \mathrm{ML}\end{array}$ & $\begin{array}{l}\text { Mina, Nevada } \\
\text { Black Star Mine } \\
\text { (USGS) }\end{array}$ & $\begin{array}{r}38.434 \mathrm{~N} \\
118.154 \mathrm{~W}\end{array}$ & (3) & (2) & & (1) & \\
\hline $\begin{array}{l}17 \text { August } 1987 \\
1428: 07.4 \text { G.m.t. } \\
\text { Central Cal if. } \\
36.555 \mathrm{~N}, 121.158 \mathrm{~W}\end{array}$ & $\begin{array}{l}\text { Bear Valley Station } 10 \\
\text { Webb Residence } \\
\text { (USGS) }\end{array}$ & $\begin{array}{r}36.532 \mathrm{~N} \\
121.143 \mathrm{~W}\end{array}$ & $28: 08.9$ & 1.1 & $\begin{array}{l}310 \\
\text { Up } \\
220\end{array}$ & $\begin{array}{l}.06 \\
.03 \\
.10\end{array}$ & 1 peak \\
\hline
\end{tabular}


Table 1. Catalogue of National Cooperative Strong-Motion Network accelerograph records recovered during 1987-Continued

\begin{tabular}{|c|c|c|c|c|c|c|c|}
\hline Earthquake & $\begin{array}{l}\text { Station name } \\
\text { (owner) }\end{array}$ & $\begin{array}{l}\text { Station } \\
\text { location } \\
\left({ }^{\circ}\right)\end{array}$ & $\begin{array}{l}\text { Trigger } \\
\text { tine }\end{array}$ & $\begin{array}{c}\text { s-minus } \\
\text { trigger } \\
(\mathrm{s})\end{array}$ & $\begin{array}{l}\text { Direction } \\
\quad(a z)\end{array}$ & $\begin{array}{l}\text { Max imun } \\
\text { ampl itude } \\
\text { (g) }\end{array}$ & $\begin{array}{l}\text { Duration } \\
(\mathrm{s})\end{array}$ \\
\hline $\begin{array}{l}25 \text { August } 1987 \\
0627: 26.6 \mathrm{GMT} \\
\text { Southern Cal if. } \\
34.360 \mathrm{~N}, 117.580 \mathrm{~W} \\
\text { Magnitude } 3.6 \mathrm{ML}\end{array}$ & $\begin{array}{l}\text { Lone Pine Canyon } \\
\text { (USGS) }\end{array}$ & $\begin{array}{r}34.32 \mathrm{~N} \\
117.57 \mathrm{~W}\end{array}$ & $27: 28.4$ & 1.3 & & (1) & \\
\hline \multirow{4}{*}{$\begin{array}{l}4 \text { December 1986- } \\
26 \text { August } 1987 \\
\text { Southern Calif. } \\
\text { Epicenters and } \\
\text { magnitudes unknown }\end{array}$} & $\begin{array}{l}\text { Diemer Filter Plant } \\
\text { (MWD) }\end{array}$ & $\begin{array}{r}33.91 \mathrm{~N} \\
117.82 \mathrm{~W}\end{array}$ & (2) & (2) & & & \\
\hline & Basement & & & & & (1) & \\
\hline & Reservoir Roof & & & & & (1) & \\
\hline & $\begin{array}{l}\text { Note: } \begin{array}{l}\text { One addition } \\
\text { on the reser }\end{array}\end{array}$ & $\begin{array}{l}\text { al record } \\
\text { voir roof }\end{array}$ & $\begin{array}{l}\text { recovered } \\
\text { at Diemer }\end{array}$ & $\begin{array}{l}\text { in the } b \\
\text { Filter } P l\end{array}$ & $\begin{array}{l}\text { basement and } \\
\text { lant. }\end{array}$ & & \\
\hline $\begin{array}{l}1 \text { September } 1987 \\
1656 \text { G.m.t. } \\
\text { Southern Calif. } \\
\text { Epicenter and } \\
\text { magnitude unknown }\end{array}$ & $\begin{array}{l}\text { Salton Sea } \\
\text { Wild life Refuge } \\
\text { (USGS) }\end{array}$ & $\begin{array}{r}33.18 \mathrm{~N} \\
115.62 \mathrm{~W}\end{array}$ & $56: 43.1$ & (2) & & (1) & \\
\hline \multirow[t]{2}{*}{$\begin{array}{l}7 \text { September } 1987 \\
1314: 56.9 \text { G.m.t. } \\
\text { Central Alaska } \\
64.955 \mathrm{~N}, 147.929 \mathrm{~W} \\
\text { Magn itude } 4.2 \mathrm{ML}\end{array}$} & $\begin{array}{l}\text { Fairbanks } \\
\text { University of Alaska } \\
\text { Duckering Hall } \\
\text { (USGS) }\end{array}$ & $\begin{array}{r}64.85 \mathrm{~N} \\
147.82 \mathrm{~W}\end{array}$ & (3) & (2) & & (1) & \\
\hline & $\begin{array}{l}\text { Fairbanks } \\
\text { Univers ity of Alaska } \\
\text { Magnetic Observatory } \\
\text { (USGS) }\end{array}$ & $\begin{array}{r}64.86 \mathrm{~N} \\
147.83 \mathrm{~W}\end{array}$ & (3) & 2.2 & & (1) & \\
\hline $\begin{array}{l}14 \text { September } 1987 \\
0712 \text { G.m.t. } \\
\text { Centrai Cal if. } \\
\text { Epicenter and } \\
\text { magnitude unknown }\end{array}$ & $\begin{array}{l}\text { Bear Valley Station } 1 \\
\text { CDF Fire Station } \\
\text { (USGS) }\end{array}$ & $\begin{array}{r}36.573 \mathrm{~N} \\
121.184 \mathrm{~W}\end{array}$ & $12: 52.8$ & (2) & & (1) & \\
\hline \multirow{4}{*}{$\begin{array}{l}1 \text { 0ctober } 1987 \\
1442: 20.0 \mathrm{G} . \mathrm{m} . \mathrm{t} . \\
\text { Southern Calif. } \\
34.060 \mathrm{~N}, 118.080 \mathrm{~W} \\
\text { Magnitude } 5.9 \mathrm{ML}\end{array}$} & $\begin{array}{l}\text { Garvey Reservoir } \\
\text { Abutment B } 1 \mathrm{dg} \text {. } \\
\text { (MWD) }\end{array}$ & $\begin{array}{r}34.05 \mathrm{~N} \\
118.11 \mathrm{~W}\end{array}$ & (3) & 0.2 & $\begin{array}{l}060 \\
U p \\
330\end{array}$ & $\begin{array}{l}.33 \\
.38 \\
.47\end{array}$ & $\begin{array}{l}4.6 \\
5.0 \\
4.3\end{array}$ \\
\hline & $\begin{array}{l}\text { Whittier Narrows Dam } \\
\text { (ACOE) }\end{array}$ & $\begin{array}{r}34.03 \mathrm{~N} \\
118.05 \mathrm{~W}\end{array}$ & (3) & 0.8 & & & \\
\hline & Crest & & & & $\begin{array}{l}033 \\
\text { Up } \\
303\end{array}$ & $\begin{array}{l}.31 \\
.19 \\
.32\end{array}$ & $\begin{array}{l}4.3 \\
3.8 \\
2.5\end{array}$ \\
\hline & Upstream & & & & $\begin{array}{l}152 \\
\text { Up } \\
062\end{array}$ & $\begin{array}{l}.31 \\
.46 \\
.24\end{array}$ & $\begin{array}{l}4.4 \\
6.2 \\
2.6\end{array}$ \\
\hline
\end{tabular}


Table 1. Catalogue of National Cooperative Strong-Motion Network accelerograph records recovered c'uring 1987-Continued

\begin{tabular}{|c|c|c|c|c|c|c|c|}
\hline Earthquake & $\begin{array}{l}\text { Station name } \\
\text { (owner) }\end{array}$ & $\begin{array}{l}\text { Station } \\
\text { location } \\
\left({ }^{\circ}\right)\end{array}$ & $\begin{array}{l}\text { Trigger } \\
\text { time }\end{array}$ & $\begin{array}{l}\text { S-minus } \\
\text { trigger } \\
(\mathrm{s})\end{array}$ & $\begin{array}{l}\text { Direction } \\
\quad(a z)\end{array}$ & $\begin{array}{l}\text { Maximum } \\
\text { ampl itude } \\
(\mathrm{g})\end{array}$ & $\begin{array}{l}\text { Duration } \\
\text { (s) }\end{array}$ \\
\hline \multirow{4}{*}{$\begin{array}{l}1 \text { October } 1987 \\
1442: 20.0 \text { G.m.t. } \\
\text { Southern Cal if. } \\
34.060 \mathrm{~N}, 118.080 \mathrm{~W} \\
\text { Magnitude } 5.9 \mathrm{ML}-- \\
\text { Cont inued }\end{array}$} & $\begin{array}{l}\text { Alhambra } \\
900 \text { S. Fremont Ave. } \\
\text { (USGS) }\end{array}$ & $\begin{array}{r}34.09 \mathrm{~N} \\
118.15 \mathrm{~W}\end{array}$ & (3) & 1.7 & & & \\
\hline & Basement & & & & $\begin{array}{l}090 \\
U p \\
360\end{array}$ & $\begin{array}{l}.30 \\
.19 \\
.26\end{array}$ & $\begin{array}{l}3.7 \\
3.2 \\
3.4\end{array}$ \\
\hline & 6th Floor & & & & $\begin{array}{l}090 \\
\text { Up } \\
360\end{array}$ & $\begin{array}{l}.47 \\
.18 \\
.37\end{array}$ & $\begin{array}{l}5.2 \\
3.8 \\
6.0\end{array}$ \\
\hline & 12th Floor & & & & $\begin{array}{l}090 \\
\text { Up } \\
360\end{array}$ & $\begin{array}{l}.28 \\
.29 \\
.23\end{array}$ & $\begin{array}{l}3.4 \\
5.5 \\
4.8\end{array}$ \\
\hline
\end{tabular}

Note: Five each additional records ${ }^{1}$ recovered from basement, 6 th and 12th floors at AThambra.

\begin{tabular}{|c|c|c|c|c|c|c|}
\hline $\begin{array}{l}\text { Whittier } \\
7215 \text { Bright Ave. } \\
\text { (USGS) }\end{array}$ & $\begin{array}{r}33.977 \mathrm{~N} \\
118.036 \mathrm{~W}\end{array}$ & (3) & 0.3 & & & \\
\hline Basement & & & & $\begin{array}{l}180 \\
\text { Up } \\
090\end{array}$ & $\begin{array}{l}.40 \\
.26 \\
.63\end{array}$ & $\begin{array}{l}1.9 \\
4.0 \\
3.0\end{array}$ \\
\hline 5 th Floor & & & & $\begin{array}{l}180 \\
\text { Up } \\
090\end{array}$ & $\begin{array}{l}.61 \\
.34 \\
.60\end{array}$ & $\begin{array}{l}3.0 \\
4.2 \\
6.6\end{array}$ \\
\hline 10th Floor & & & & $\begin{array}{l}180 \\
\text { Up } \\
090\end{array}$ & $\begin{array}{l}.41 \\
.54 \\
.54\end{array}$ & $\begin{array}{l}3.5 \\
6.0 \\
6.3\end{array}$ \\
\hline $\begin{array}{l}\text { Los Angeles } \\
4407 \text { Jasper Street } \\
\text { (USGS) }\end{array}$ & $\begin{array}{r}34.081 \mathrm{~N} \\
118.188 \mathrm{~W}\end{array}$ & (3) & (2) & $\begin{array}{l}130 \\
\text { Up } \\
040\end{array}$ & $\begin{array}{l}.33 \\
.13 \\
.23\end{array}$ & $\begin{array}{l}2.8 \\
2.1 \\
2.9\end{array}$ \\
\hline
\end{tabular}

Note: Partial record, film fogged for approx. the first $3 \mathrm{sec}$. after triggering.

\begin{tabular}{|c|c|c|c|c|c|}
\hline $\begin{array}{l}\text { Los Angeles } \\
\text { Bulk Mail Center } \\
\text { (USGS) }\end{array}$ & $\begin{array}{r}33.99 \mathrm{~N} \\
118.16 \mathrm{~W}\end{array}$ & (3) & 0.9 & $\begin{array}{l}010 \\
\text { Up } \\
280\end{array}$ & $\begin{array}{l}.34 \\
.52 \\
.46\end{array}$ \\
\hline $\begin{array}{l}\text { Vernon } \\
4814 \text { Loma Vista Ave. } \\
\text { (USGS) }\end{array}$ & $\begin{array}{r}34.00 \mathrm{~N} \\
118.20 \mathrm{~W}\end{array}$ & (3) & 2.9 & $\begin{array}{l}007 \\
\text { Up } \\
277\end{array}$ & $\begin{array}{l}.29 \\
.17 \\
.22\end{array}$ \\
\hline $\begin{array}{l}\text { Morris Dam } \\
\text { Left Abutment } \\
\text { (MWD) }\end{array}$ & $\begin{array}{r}34.17 \mathrm{~N} \\
117.88 \mathrm{~W}\end{array}$ & (3) & (2) & $\begin{array}{l}246 \\
\text { Up } \\
156\end{array}$ & $\begin{array}{l}.04 \\
.04 \\
.05\end{array}$ \\
\hline
\end{tabular}


Table 1. Catalogue of National Cooperative Strong-Motion Network accelerograph records recovered during 1987-Continued

\begin{tabular}{|c|c|c|c|c|c|c|c|}
\hline Earthquake & $\begin{array}{l}\text { Station name } \\
\text { (owner) }\end{array}$ & $\begin{array}{l}\text { Station } \\
\text { location } \\
\left({ }^{\circ}\right)\end{array}$ & $\begin{array}{c}\text { Trigger } \\
\text { time }\end{array}$ & $\begin{array}{c}\text { S-minus } \\
\text { trigger } \\
\text { (s) }\end{array}$ & $\begin{array}{l}\text { Direction } \\
\quad(a z)\end{array}$ & $\begin{array}{l}\text { Maximum } \\
\text { amplitude } \\
(\mathrm{g})\end{array}$ & $\begin{array}{l}\text { Duration } \\
\text { (s) }\end{array}$ \\
\hline \multirow{5}{*}{$\begin{array}{l}\text { 1. October } 1987 \\
1442: 20.0 \text { G.m.t. } \\
\text { Southern Cal if. } \\
34.060 \mathrm{~N}, 118.080 \mathrm{~W} \\
\text { Magnitude } 5.9 \mathrm{ML}-- \\
\text { Continued }\end{array}$} & $\begin{array}{l}\text { Norwalk, } 12400 \\
\text { Imperiail Highway } \\
\text { (USGS/BECH) }\end{array}$ & $\begin{array}{r}33.92 \mathrm{~N} \\
118.07 \mathrm{~W}\end{array}$ & (3) & 2.4 & & & \\
\hline & Basement & & & & $\begin{array}{l}090 \\
\text { Up } \\
360\end{array}$ & $\begin{array}{l}.12 \\
.07 \\
.21\end{array}$ & \begin{tabular}{c}
1 peak \\
\hdashline 1.6
\end{tabular} \\
\hline & 4th Floor & & & & $\begin{array}{l}090 \\
\text { Up } \\
360\end{array}$ & $\begin{array}{l}.17 \\
.12 \\
.33\end{array}$ & $\begin{array}{c}0.4 \\
1 \text { peak } \\
4.7\end{array}$ \\
\hline & Roof & & & & $\begin{array}{l}090 \\
\text { Up } \\
360\end{array}$ & $\begin{array}{l}.21 \\
.18 \\
.40\end{array}$ & $\begin{array}{l}5.9 \\
2.8 \\
6.7\end{array}$ \\
\hline & South Ground Site & & & & $\begin{array}{l}090 \\
\text { Up } \\
360\end{array}$ & $\begin{array}{l}.10 \\
.09 \\
.29\end{array}$ & $\begin{array}{l}1 \text { peak } \\
--- \\
2.0\end{array}$ \\
\hline
\end{tabular}

Note: Two additional records ${ }^{1}$ recovered at south ground site.

\begin{tabular}{|c|c|c|}
\hline $\begin{array}{l}\text { Norwalk, } 12440 \\
\text { Imperial Highway } \\
\text { (USGS/BECH) }\end{array}$ & $\begin{array}{r}33.92 \mathrm{~N} \\
118.07 \mathrm{~W}\end{array}$ & $42: 24.7$ \\
\hline
\end{tabular}

Basement

North Ground Site

South Ground Site

Structure Array 1:

Ch. 1- 8th Floor (Roof) Center

Ch. 2- 5th Floor, Center

Ch. 3- 2nd Floor, Center

Ch. 4- 1st Floor, Center

Ch. 5- Basement, East

Ch. 6- 5th Floor, West-Center

Ch. 7- Basement, Center

Ch. 8- Basement, Center

Ch. 9- Basement, Center

Ch. $10-30 \mathrm{ft}$. Downhole, B $1 \mathrm{dg}$. Center

Ch. $11-30 \mathrm{ft}$. Downhole, B Tdg. Center

Ch. 12- $30 \mathrm{ft}$. Downhole, Bldg. Center

$\begin{array}{lll}090 & .11 & 1 \text { peak } \\ \text { Up } & .10 & 1 \text { peak } \\ 360 & .21 & 1.3 \\ & & \\ 090 & .13 & 0.4 \\ \text { Up } & .13 & 0.2 \\ 360 & .24 & 1.3 \\ 090 & .09 & --- \\ \text { Up } & .10 & 1 \text { peak } \\ 360 & .21 & 1.3\end{array}$

090

090

090

090

360

360

Up

090

360

Up

090

360
.18

.15

.12

.13

.19

.23

.05

.11

.20

.05

.10

.16
7.1

2 peaks

1.0

0.7

8.7

$---$

0.9

$---$

0.2 
Table 1. Catalogue of National Cooperative Strong-Motion Network accelerograph records recovered during 1987-Continued

\begin{tabular}{llllll}
\hline Earthquake & $\begin{array}{c}\text { Station name } \\
\text { (owner) }\end{array}$ & $\begin{array}{c}\text { Station } \\
\text { location } \\
\left({ }^{\circ}\right)\end{array}$ & $\begin{array}{c}\text { Trigger } \\
\text { time }\end{array}$ & $\begin{array}{c}\text { S-minus Direction Maximum } \\
\text { trigger } \\
(\mathrm{s})\end{array}$ & $\begin{array}{c}\text { Duration } \\
(\mathrm{az})\end{array}$ \\
\hline
\end{tabular}

1 October $1987 \quad$ Norwalk, 12440--Continued

1442:20.0 G.m.t.

Southern Calif.

$34.060 \mathrm{~N}, 118.080 \mathrm{~W}$

Magnitude 5.9 ML--

Structure Array 2:

Ch. 13- 8th Floor (Roof), East

Ch. 14- 5th Floor, East

Ch. 15- 2nd Floor, East

Ch. 16- 1st Floor, East

Ch. 17-8th Floor (Roof), Center

Ch. 18- 5th Floor, Center

Ch. 19- 2nd Floor, Center

Ch. 20- 1st Floor, Center

Ch. 21- 8th Floor (Roof), west

Ch. 22- 5th Floor, West

Ch. 23- 2nd Floor, West

Ch. 24- 1st Floor, West

$\begin{array}{rrr}360 & .31 & 9.5 \\ 360 & .14 & 0.3 \\ 360 & .17 & 0.5 \\ 360 & .18 & 1.1 \\ 360 & .41 & 14.7 \\ 360 & .26 & -.- \\ 360 & .30 & 2.1 \\ 360 & .23 & 1.6 \\ 360 & .27 & 11.2 \\ 360 & .12 & 1.7 \\ 360 & .19 & 0.5 \\ 360 & .22 & 1.4\end{array}$

Note: Channel 18 failed after $2.5 \mathrm{sec}$.

\begin{tabular}{|c|c|c|c|c|}
\hline $\begin{array}{l}\text { Los Angeles } \\
1111 \text { Sunset Blvd. } \\
\text { (MWD) }\end{array}$ & $\begin{array}{r}34.07 \mathrm{~N} \\
118.25 \mathrm{~W}\end{array}$ & 2.4 & & \\
\hline Basement & & $\begin{array}{l}348 \\
\text { Up } \\
258\end{array}$ & $\begin{array}{l}.16 \\
.07 \\
.11\end{array}$ & $\begin{array}{l}2.2 \\
0.7\end{array}$ \\
\hline 4th Floor & & $\begin{array}{l}348 \\
\text { Up } \\
258\end{array}$ & $\begin{array}{l}.19 \\
.09 \\
.11\end{array}$ & $\begin{array}{c}4.0 \\
-1 \text { peak }\end{array}$ \\
\hline Roof & & $\begin{array}{l}348 \\
\text { Up } \\
258\end{array}$ & $\begin{array}{l}.18 \\
.22 \\
.15\end{array}$ & $\begin{array}{l}3.8 \\
3.8 \\
1.9\end{array}$ \\
\hline
\end{tabular}

Note: Three each additional records ${ }^{1}$ recovered from basement, 4 th floor and roof at 1111 Sunset Boulevard.

\begin{tabular}{|c|c|c|c|c|c|}
\hline $\begin{array}{l}\text { Los Angeles } \\
\text { Griffith Park Observ. } \\
\text { (USGS) }\end{array}$ & $\begin{array}{r}34.12 \mathrm{~N} \\
118.30 \mathrm{~W}\end{array}$ & (3) & 2.3 & $\begin{array}{l}360 \\
\text { Up } \\
270\end{array}$ & $\begin{array}{l}.13 \\
.06 \\
.15\end{array}$ \\
\hline
\end{tabular}

Note: One additional record ${ }^{1}$ recovered at Griffith Park Observatory.

$\begin{array}{lrrr}\text { Orange County Resvr. } & 33.935 \mathrm{~N} & \text { (3) } & 1.2 \\ \text { (MWD) } & 117.883 \mathrm{~W} & & \end{array}$

Abutment

$\begin{array}{lll}096 & .23 & 1.7 \\ \text { Up } & .10 & 0.2 \\ 006 & .21 & 1.5\end{array}$

Long Beach, CSULB $\quad 33.777 \mathrm{~N}$

(3)

$118.112 \mathrm{~W}$

$\begin{array}{ll}4.5 & 104 \\ & \text { Up } \\ & 014\end{array}$

$\begin{array}{ll}.09 & --- \\ .05 & -- \\ .09 & --\end{array}$ 
Table 1. Catalogue of National Cooperative Strong-Motion Network accelerograph records recovered during 1987-Continued

\begin{tabular}{|c|c|c|c|c|c|c|c|}
\hline Earthquake & $\begin{array}{l}\text { Station name } \\
\text { (owner) }\end{array}$ & $\begin{array}{l}\text { Station } \\
\text { location } \\
\left({ }^{\circ}\right)\end{array}$ & $\begin{array}{l}\text { Trigger } \\
\text { time }\end{array}$ & $\begin{array}{c}\text { S-minus } \\
\text { trigger } \\
(\mathrm{s})\end{array}$ & $\begin{array}{l}\text { Direction } \\
\quad(a z)\end{array}$ & $\begin{array}{l}\text { Maximum } \\
\text { ampl itudo } \\
\text { (g) }\end{array}$ & $\begin{array}{l}\text { Duration } \\
\text { (s) }\end{array}$ \\
\hline \multirow{5}{*}{$\begin{array}{l}1 \text { october } 1987 \\
1442: 20.0 \text { G.m.t. } \\
\text { Southern Calif. } \\
34.060 \mathrm{~N}, 118.080 \mathrm{~W} \\
\text { Magnitude } 5.9 \mathrm{ML}-- \\
\text { Continued }\end{array}$} & $\begin{array}{l}\text { Los Angeles } \\
1100 \text { Wilshire Blvd. } \\
\text { (JCG/USGS) }\end{array}$ & $\begin{array}{r}34.052 \mathrm{~N} \\
118.263 \mathrm{~W}\end{array}$ & $42: 24.5$ & 2.9 & & & \\
\hline & Basement 3 , NE & & & & $\begin{array}{l}298 \\
\text { Up } \\
208\end{array}$ & $\begin{array}{l}.18 \\
.07 \\
.12\end{array}$ & \begin{tabular}{l}
1.1 \\
\hdashline 1.3
\end{tabular} \\
\hline & Basement $3, \mathrm{SE}$ & & & & $\begin{array}{l}298 \\
\text { Up } \\
208\end{array}$ & $\begin{array}{l}.18 \\
.07 \\
.11\end{array}$ & $\begin{array}{l}0.8 \\
-.- \\
0.2\end{array}$ \\
\hline & Basement 4 , NW & & & & $\begin{array}{l}298 \\
\text { Up } \\
208\end{array}$ & $\begin{array}{l}.17 \\
.08 \\
.11\end{array}$ & $\begin{array}{c}1.0 \\
-- \\
1 \text { peak }\end{array}$ \\
\hline & $\begin{array}{l}\text { Structure Array: } \\
\text { Ch. 1- 12th Floor, } \\
\text { Ch. 2- 12th Floor, } \\
\text { Ch. 3- 12th Floor, } \\
\text { Ch. 4- 13th Floor, } \\
\text { Ch. 5-13th Floor, } \\
\text { Ch. 6- 13th Floor, } \\
\text { Ch. 7-32nd Floor, } \\
\text { Ch. 8- 32nd Floor, } \\
\text { Ch. 9-32nd Floor, } \\
\text { Ch. 10- Ground Flo } \\
\text { Ch. 11- Ground Flo } \\
\text { Ch. 12- Ground Flo }\end{array}$ & $\begin{array}{l}\text { North } \\
\text {, North } \\
\text {, South } \\
\text {, North } \\
\text {, North } \\
\text {, South } \\
\text {, North } \\
\text {, North } \\
\text {, South } \\
\text { oor, North } \\
\text { oor, North } \\
\text { oor, South }\end{array}$ & & & $\begin{array}{l}298 \\
208 \\
208 \\
298 \\
208 \\
208 \\
298 \\
208 \\
208 \\
298 \\
208 \\
208\end{array}$ & $\begin{array}{l}.13 \\
.24 \\
.10 \\
.14 \\
.26 \\
.12 \\
.17 \\
.19 \\
.10 \\
.17 \\
.21 \\
.15\end{array}$ & $\begin{array}{l}2 \text { peaks } \\
1.2 \\
0.4 \\
3 \text { peaks } \\
3.2 \\
2 \text { peaks } \\
1.6 \\
5.2 \\
1 \text { peak } \\
1.8 \\
1.7 \\
2 \text { peaks }\end{array}$ \\
\hline
\end{tabular}

$\begin{array}{lr}\text { Brea Dam } & 33.889 \mathrm{~N} \\ \text { (ACOE) } & 117.926 \mathrm{~W} \\ \text { Crest } & \end{array}$

Left Abutment

Downstream
(3)

4.0
130
Up
040

.31

.14

.23

130

Up

040

130

Up

.16

.09

.09

2.5

1.7

2.5

1.3

.101 peak

$.32 \quad 1.1$

-..

$.18 \quad 0.6$

Note: One additional record ${ }^{1}$ recovered at Brea Dam crest.

\begin{tabular}{|c|c|c|c|c|c|c|}
\hline $\begin{array}{l}\text { Lawndale } \\
15000 \text { Aviation Blvd. } \\
\text { (USGS) }\end{array}$ & $\begin{array}{r}33.895 \mathrm{~N} \\
118.377 \mathrm{~W}\end{array}$ & (3) & 4.1 & $\begin{array}{l}360 \\
\text { Up } \\
270\end{array}$ & $\begin{array}{l}.06 \\
.04 \\
.06\end{array}$ & --. \\
\hline $\begin{array}{l}\text { Santa Ana, Orange Cty. } \\
\text { Eng ineering B Idg., } \\
\text { Basement (USGS) }\end{array}$ & $\begin{array}{r}33.75 \mathrm{~N} \\
117.87 \mathrm{~W}\end{array}$ & (3) & 0.5 & $\begin{array}{l}360 \\
\text { Up } \\
270\end{array}$ & $\begin{array}{l}.09 \\
.03 \\
.05\end{array}$ & \\
\hline
\end{tabular}


Table 1. Catalogue of National Cooperative Strong-Motion Network accelerograph records recovered during 1987-Continued

\begin{tabular}{|c|c|c|c|c|c|c|c|}
\hline Earthquake & $\begin{array}{l}\text { Station name } \\
\text { (owner) }\end{array}$ & $\begin{array}{l}\text { Station } \\
\text { location } \\
\left({ }^{\circ}\right)\end{array}$ & $\begin{array}{l}\text { Trigger } \\
\text { time }\end{array}$ & $\begin{array}{l}\text { S-minus } \\
\text { trigger } \\
(\mathrm{s})\end{array}$ & $\begin{array}{l}\text { Direction } \\
\quad(a z)\end{array}$ & $\begin{array}{l}\text { Maximum } \\
\text { amplitude } \\
\text { (g) }\end{array}$ & $\begin{array}{l}\text { Duration } \\
\text { (s) }\end{array}$ \\
\hline \multirow{3}{*}{$\begin{array}{l}1 \text { October } 1987 \\
1442: 20.0 \text { G.m.t. } \\
\text { Southern Calif. } \\
34.060 \mathrm{~N}, 118.080 \mathrm{~W} \\
\text { Magnitude } 5.9 \mathrm{ML}-- \\
\text { Continued }\end{array}$} & $\begin{array}{l}\text { Carbon Canyon Dam } \\
\text { (ACOE) }\end{array}$ & $\begin{array}{r}33.92 \mathrm{~N} \\
117.84 \mathrm{~W}\end{array}$ & (3) & 4.6 & & & \\
\hline & Crest & & & & $\begin{array}{l}130 \\
\text { Up } \\
040\end{array}$ & $\begin{array}{l}.17 \\
.13 \\
.20\end{array}$ & $\begin{array}{l}2.4 \\
0.6 \\
3.4\end{array}$ \\
\hline & Left Abutment & & & & $\begin{array}{l}130 \\
\text { Up } \\
040\end{array}$ & $\begin{array}{l}.22 \\
.07 \\
.16\end{array}$ & $\begin{array}{l}1.8 \\
--- \\
2.6\end{array}$ \\
\hline
\end{tabular}

Note: Three additional records ${ }^{1}$ recovered at Carbon Canyon Dam crest.

\begin{tabular}{|c|c|c|c|c|c|c|}
\hline $\begin{array}{l}\text { Weymouth Filter Plant } \\
\text { (MWD) }\end{array}$ & $\begin{array}{r}34.114 \mathrm{~N} \\
117.778 \mathrm{~W}\end{array}$ & (3) & 3.4 & & & \\
\hline B1dg., Ground Level & & & & $\begin{array}{l}017 \\
\text { Up } \\
287\end{array}$ & $\begin{array}{l}.10 \\
.05 \\
.06\end{array}$ & $\begin{array}{c}1 \text { peak } \\
---\end{array}$ \\
\hline Water Tank, Top & & & & $\begin{array}{l}017 \\
\text { Up } \\
287\end{array}$ & $\begin{array}{l}.15 \\
.11 \\
.13\end{array}$ & $\begin{array}{l}4.6 \\
0.3 \\
1.3\end{array}$ \\
\hline $\begin{array}{l}\text { Diemer Filter Plant } \\
\text { (MWD) }\end{array}$ & $\begin{array}{r}33.91 \mathrm{~N} \\
117.82 \mathrm{~W}\end{array}$ & (3) & 4.0 & & & \\
\hline \multicolumn{2}{|l|}{ Admin. Bldg. Basement } & & & $\begin{array}{l}281 \\
\text { Up } \\
191\end{array}$ & $\begin{array}{l}.09 \\
.04 \\
.06\end{array}$ & $\begin{array}{l}--- \\
--- \\
---\end{array}$ \\
\hline Reservoir Roof & & & & $\begin{array}{l}281 \\
\text { Up } \\
191\end{array}$ & $\begin{array}{l}.11 \\
.17 \\
.11\end{array}$ & $\begin{array}{c}1 \text { peak } \\
0.3 \\
1 \text { peak }\end{array}$ \\
\hline
\end{tabular}

Note: Two each additional records ${ }^{1}$ recovered from the administration building and reservoir roof at Diemer filter plant.

\begin{tabular}{|c|c|c|c|c|c|c|}
\hline $\begin{array}{l}\text { Palos Verdes Estates } \\
\text { Basement } \\
\text { (USGS) }\end{array}$ & $\begin{array}{r}33.801 \mathrm{~N} \\
118.387 \mathrm{~W}\end{array}$ & (3) & 1.1 & & (1) & \\
\hline $\begin{array}{l}\text { Sepulveda Canyon } \\
\text { Sp } i 11 \text { way Roof } \\
\text { (MWD) }\end{array}$ & $\begin{array}{r}34.097 \mathrm{~N} \\
118.478 \mathrm{~W}\end{array}$ & (3) & 0.3 & $\begin{array}{l}166 \\
\text { Up } \\
076\end{array}$ & $\begin{array}{l}.03 \\
.02 \\
.08\end{array}$ & $\begin{array}{l}--- \\
---\end{array}$ \\
\hline $\begin{array}{l}\text { Live 0ak Reservoir } \\
\text { (MWD) }\end{array}$ & $\begin{array}{r}34.134 \mathrm{~N} \\
117.753 \mathrm{~W}\end{array}$ & (3) & (2) & & & \\
\hline Abutment & & & & $\begin{array}{l}180 \\
\text { Up } \\
090\end{array}$ & $\begin{array}{l}.04 \\
.02 \\
.03\end{array}$ & $\begin{array}{l}--- \\
--- \\
---\end{array}$ \\
\hline
\end{tabular}


Table 1. Catalogue of National Cooperative Strong-Motion Network accelerograph records recovered during 1987 -Continued

\begin{tabular}{|c|c|c|c|c|c|c|c|}
\hline Earthquake & $\begin{array}{l}\text { Station name } \\
\text { (owner) }\end{array}$ & $\begin{array}{l}\text { Station } \\
\text { location } \\
\left({ }^{\circ}\right)\end{array}$ & $\begin{array}{l}\text { Trigger } \\
\text { time }\end{array}$ & $\begin{array}{l}\text { S-minus } \\
\text { trigger } \\
\text { (s) }\end{array}$ & $\begin{array}{l}\text { Direction } \\
\quad(a z)\end{array}$ & $\begin{array}{l}\text { Maximur } \\
\text { amplitude } \\
\text { (g) }\end{array}$ & $\begin{array}{l}\text { Duration } \\
\text { (s) }\end{array}$ \\
\hline \multirow[t]{9}{*}{$\begin{array}{l}1 \text { 0ctober } 1987 \\
1442: 20.0 \text { G.m.t. } \\
\text { Southern Calif. } \\
34.060 \mathrm{~N}, 118.080 \mathrm{~W} \\
\text { Magnitude } 5.9 \mathrm{ML-}- \\
\text { Continued }\end{array}$} & \multicolumn{4}{|c|}{$\begin{array}{l}\text { Live 0ak Reservoir--Continued } \\
\text { Structure Array: } \\
\text { Ch. 1- Center Crest } \\
\text { Ch. 2- Center Crest } \\
\text { Ch. 3- Center Crest } \\
\text { Ch. 4- Left Crest } \\
\text { Ch. 5- Left Crest } \\
\text { Ch. 6- Left Slope } \\
\text { Ch. 7- Center Slope } \\
\text { Ch. 8- Center Slope } \\
\text { Ch. 9- Center Slope } \\
\text { Ch. 10- Center Toe } \\
\text { Ch. 11- Center Toe } \\
\text { Ch. 12- Center Toe }\end{array}$} & $\begin{array}{l}155 \\
\text { Up } \\
245 \\
155 \\
245 \\
245 \\
155 \\
\text { Up } \\
245 \\
155 \\
\text { Up } \\
245\end{array}$ & $\begin{array}{l}.07 \\
.04 \\
.07 \\
.09 \\
.08 \\
.04 \\
.09 \\
.04 \\
.06 \\
.05 \\
.02 \\
.03\end{array}$ & $\begin{array}{l}--- \\
--- \\
--- \\
--- \\
--- \\
--- \\
--- \\
--- \\
--- \\
--- \\
--- \\
---\end{array}$ \\
\hline & $\begin{array}{l}\text { Long Beach } \\
\text { VA Hospital } \\
\text { (VA) }\end{array}$ & \multirow[t]{2}{*}{$\begin{array}{r}33.78 \mathrm{~N} \\
118.12 \mathrm{~W}\end{array}$} & \multirow[t]{2}{*}{ (3) } & \multirow[t]{2}{*}{4.6} & & & \\
\hline & Basement & & & & $\begin{array}{l}360 \\
\text { Up } \\
270\end{array}$ & $\begin{array}{l}.08 \\
.05 \\
.10\end{array}$ & $1 \overline{---}$ \\
\hline & 6th Floor & & & & $\begin{array}{l}360 \\
\text { Up } \\
270\end{array}$ & $\begin{array}{l}.17 \\
.08 \\
.11\end{array}$ & $\begin{array}{c}1.2 \\
-- \\
1 \text { peak }\end{array}$ \\
\hline & 11th Floor & & & & $\begin{array}{l}360 \\
\text { Up } \\
270\end{array}$ & $\begin{array}{l}.26 \\
.11 \\
.16\end{array}$ & $\begin{array}{l}2.1 \\
1 \text { peak } \\
2.1\end{array}$ \\
\hline & $\begin{array}{l}\text { Los Ange les } \\
\text { WadSworth VA Hospital } \\
\text { (VA/USGS) }\end{array}$ & $\begin{array}{r}34.050 \mathrm{~N} \\
118.452 \mathrm{~W}\end{array}$ & (3) & 0.5 & & & \\
\hline & \multicolumn{4}{|c|}{$\begin{array}{l}\text { Bldg. } 500 \text { Structure Array: } \\
\text { Ch. 1-6th Floor, North End } \\
\text { Ch. 2- 6th Floor, North Center } \\
\text { Ch. 3- 6th Floor, Center } \\
\text { Ch. 4-6th Floor, Center } \\
\text { Ch. 5- 6th Floor, South End } \\
\text { Ch. 6- 6th Floor, South End } \\
\text { Ch. 7- Basement, North Center } \\
\text { Ch. 8- Basement, North Center } \\
\text { Ch. 9- Basement, North Center }\end{array}$} & $\begin{array}{l}235 \\
235 \\
235 \\
055 \\
055 \\
325 \\
325 \\
235 \\
\text { Down }\end{array}$ & $\begin{array}{l}.20 \\
.15 \\
.15 \\
.18 \\
.25 \\
.13 \\
.07 \\
.07 \\
.04\end{array}$ & $\begin{array}{r}14.3 \\
4.3 \\
3.1 \\
4.1 \\
4.0 \\
1.1 \\
--- \\
--- \\
---\end{array}$ \\
\hline & \multicolumn{2}{|l|}{ North Ground Site } & $42: 27.4$ & 4.7 & $\begin{array}{l}325 \\
\text { Up } \\
235\end{array}$ & $\begin{array}{l}.07 \\
.03 \\
.08\end{array}$ & $\begin{array}{l}--- \\
--- \\
---\end{array}$ \\
\hline & \multicolumn{2}{|l|}{ South Ground Site } & $42: 28.1$ & 4.5 & $\begin{array}{l}325 \\
\text { Up } \\
235\end{array}$ & $\begin{array}{l}.07 \\
.04 \\
.09\end{array}$ & $\begin{array}{l}--- \\
---\end{array}$ \\
\hline
\end{tabular}


Table 1. Catalogue of National Cooperative Strong-Motion Network accelerograph records recovered during 1987-Continued

\begin{tabular}{|c|c|c|c|c|c|c|c|}
\hline Earthquake & $\begin{array}{l}\text { Station name } \\
\text { (owner) }\end{array}$ & $\begin{array}{l}\text { Station } \\
\text { location } \\
\left({ }^{\circ}\right)\end{array}$ & $\begin{array}{l}\text { Trigger } \\
\text { time }\end{array}$ & $\begin{array}{l}\text { S-minus } \\
\text { trigger } \\
(\mathrm{s})\end{array}$ & $\begin{array}{l}\text { Direct ion } \\
\quad(a z)\end{array}$ & $\begin{array}{l}\text { Maximum } \\
\text { amp Titude } \\
(\mathrm{g})\end{array}$ & $\begin{array}{l}\text { Duration } \\
\text { (s) }\end{array}$ \\
\hline \multirow{8}{*}{$\begin{array}{l}1 \text { october } 1987 \\
1442: 20.0 \text { G.m.t. } \\
\text { Southern Cai if. } \\
34.060 \mathrm{~N}, 118.080 \mathrm{~W} \\
\text { Magnitude } 5.9 \mathrm{ML}-- \\
\text { Continued }\end{array}$} & $\begin{array}{l}\text { Los Angeles, Brentwood } \\
\text { VA Hospital } \\
\text { (VA) }\end{array}$ & $\begin{array}{r}34.058 \mathrm{~N} \\
118.457 \mathrm{~W}\end{array}$ & (3) & 4.1 & $\begin{array}{l}285 \\
\text { Up } \\
195\end{array}$ & $\begin{array}{l}.04 \\
.03 \\
.05\end{array}$ & $\begin{array}{l}--- \\
--- \\
--\end{array}$ \\
\hline & $\begin{array}{l}\text { San Antonio Dam } \\
(A C O E)\end{array}$ & $\begin{array}{r}34.166 \mathrm{~N} \\
117.680 \mathrm{~W}\end{array}$ & (3) & 3.1 & & & \\
\hline & Crest & & & & $\begin{array}{l}090 \\
\text { Up } \\
360\end{array}$ & $\begin{array}{l}.10 \\
.06 \\
.14\end{array}$ & $\begin{array}{l}2 \text { peaks } \\
-1.9\end{array}$ \\
\hline & Right Abutment & & & & $\begin{array}{l}090 \\
U p \\
360\end{array}$ & $\begin{array}{l}.04 \\
.05 \\
.04\end{array}$ & $\begin{array}{l}--- \\
---\end{array}$ \\
\hline & Downstream & & & & $\begin{array}{l}090 \\
\text { Up } \\
360\end{array}$ & $\begin{array}{l}.07 \\
.04 \\
.05\end{array}$ & $\begin{array}{l}--- \\
--- \\
--\end{array}$ \\
\hline & $\begin{array}{l}\text { Sepulveda Dam } \\
\text { (ACOE) }\end{array}$ & $\begin{array}{r}34.17 \mathrm{~N} \\
118.47 \mathrm{~W}\end{array}$ & (3) & 4.9 & & & \\
\hline & Crest & & & & $\begin{array}{l}054 \\
\text { Up } \\
324\end{array}$ & $\begin{array}{l}.09 \\
.09 \\
.14\end{array}$ & 1 peak \\
\hline & Downstream & & & & $\begin{array}{l}054 \\
\text { Up } \\
324\end{array}$ & $\begin{array}{l}.12 \\
.07 \\
.15\end{array}$ & $\begin{array}{l}1 \text { peak } \\
1 \text { peak }\end{array}$ \\
\hline
\end{tabular}

Note: One additional record ${ }^{1}$ recovered at Sepulveda Dam downstream.

\begin{tabular}{|c|c|c|c|c|c|}
\hline $\begin{array}{l}\text { Palos Verdes Reservoir } \\
\text { Abutment } \\
\text { (MWD) }\end{array}$ & $\begin{array}{r}33.774 \mathrm{~N} \\
118.321 \mathrm{~W}\end{array}$ & (3) & 2.2 & & (1) \\
\hline $\begin{array}{l}\text { SepuTveda VA Hospital } \\
\text { Ground level } \\
\text { (VA) }\end{array}$ & $\begin{array}{r}34.249 \mathrm{~N} \\
118.475 \mathrm{~W}\end{array}$ & (3) & 5.7 & $\begin{array}{l}360 \\
\text { Up } \\
270\end{array}$ & $\begin{array}{l}.18 \\
.10 \\
.22\end{array}$ \\
\hline
\end{tabular}

Note: Two additional records ${ }^{1}$ recovered at Sepulveda VA Hospital.

\begin{tabular}{|c|c|c|c|c|c|}
\hline $\begin{array}{l}\text { Valyermo Forest Sta. } \\
\text { Ground Level } \\
\text { (USGS) }\end{array}$ & $\begin{array}{r}34.44 \mathrm{~N} \\
117.85 \mathrm{~W}\end{array}$ & $42: 29.3$ & 4.7 & $\begin{array}{l}300 \\
\text { Up } \\
210\end{array}$ & $\begin{array}{l}.04 \\
.04 \\
.05\end{array}$ \\
\hline $\begin{array}{l}\text { Topanga Fire Station } \\
\text { (USGS) }\end{array}$ & $\begin{array}{r}34.084 \mathrm{~N} \\
118.600 \mathrm{~W}\end{array}$ & $42: 35.2$ & (2) & & (1) \\
\hline $\begin{array}{l}\text { Paradise Springs Camp } \\
\text { Ground Level } \\
\text { (USGS) }\end{array}$ & $\begin{array}{r}34.40 \mathrm{~N} \\
117.80 \mathrm{~W}\end{array}$ & $42: 34.1$ & (2) & & (1) \\
\hline
\end{tabular}


Table 1. Catalogue of National Cooperative Strong-Motion Network accelerograph records recovered during 1987-Continued

\begin{tabular}{|c|c|c|c|c|c|c|c|}
\hline Earthquake & $\begin{array}{l}\text { Station name } \\
\text { (owner) }\end{array}$ & $\begin{array}{l}\text { Station } \\
\text { location } \\
\left({ }^{\circ}\right)\end{array}$ & $\begin{array}{l}\text { Trigger } \\
\text { time }\end{array}$ & $\begin{array}{l}\text { S-minus } \\
\text { trigger } \\
(\mathrm{s})\end{array}$ & $\begin{array}{l}\text { Direction } \\
\quad(a z)\end{array}$ & $\begin{array}{l}\text { Maximum } \\
\text { amplitude } \\
\text { (g) }\end{array}$ & $\begin{array}{l}\text { Duration } \\
\text { (s) }\end{array}$ \\
\hline \multirow{14}{*}{$\begin{array}{l}1 \text { October } 1987 \\
1442: 20.0 \text { G.m.t. } \\
\text { Southern Calif. } \\
34.060 \mathrm{~N}, 118.080 \mathrm{~W} \\
\text { Magnitude } 5.9 \mathrm{ML}-- \\
\text { Continued }\end{array}$} & $\begin{array}{l}\text { Prado Dam } \\
\text { (ACOE) }\end{array}$ & $\begin{array}{r}33.89 \mathrm{~N} \\
117.64 \mathrm{~W}\end{array}$ & (3) & 5.4 & & & \\
\hline & Crest & & & & $\begin{array}{l}090 \\
\text { Up } \\
360\end{array}$ & $\begin{array}{l}.09 \\
.06 \\
.09\end{array}$ & $\begin{array}{l}--- \\
--- \\
---\end{array}$ \\
\hline & Left Abutment & & & & $\begin{array}{l}090 \\
\text { Up } \\
360\end{array}$ & $\begin{array}{l}.04 \\
.03 \\
.07\end{array}$ & --- \\
\hline & Downstream & & & & $\begin{array}{l}090 \\
\text { Up } \\
360\end{array}$ & $\begin{array}{l}.14 \\
.06 \\
.12\end{array}$ & $\begin{array}{l}0.6 \\
--. \\
1.0\end{array}$ \\
\hline & $\begin{array}{l}\text { Jensen Filter Plant } \\
\text { (MWD) }\end{array}$ & $\begin{array}{r}34.309 \mathrm{~N} \\
118.499 \mathrm{~W}\end{array}$ & (3) & 6.8 & & & \\
\hline & $\begin{array}{l}\text { Admin istration } B 1 \mathrm{dg} . \\
\text { Basement }\end{array}$ & & & & $\begin{array}{l}022 \\
\text { Up } \\
292\end{array}$ & $\begin{array}{l}.06 \\
.05 \\
.11\end{array}$ & $\begin{array}{l}--- \\
1 \text { peak }\end{array}$ \\
\hline & Generator Room & & & & $\begin{array}{l}022 \\
\text { Up } \\
292\end{array}$ & $\begin{array}{l}.09 \\
.06 \\
.16\end{array}$ & 2 peaks \\
\hline & Reservoir Roof & & & & $\begin{array}{l}022 \\
\text { Up } \\
292\end{array}$ & $\begin{array}{l}.22 \\
.10 \\
.31\end{array}$ & $\begin{array}{l}1.3 \\
1 \text { peak } \\
1.6\end{array}$ \\
\hline & $\begin{array}{ll}\text { Note: } & \text { Four each add } \\
\text { building and } \\
\text { Filter plant. }\end{array}$ & $\begin{array}{l}\text { ditional } r \\
\text { generator }\end{array}$ & $\begin{array}{l}\text { cords }{ }^{1} \text { rec } \\
\text { room and } 3\end{array}$ & $\begin{array}{l}\text { Sovered a } \\
\text { at the }\end{array}$ & $\begin{array}{l}\text { at the admi } \\
\text { reservoir }\end{array}$ & $\begin{array}{l}\text { inistration } \\
\text { roof at Je }\end{array}$ & ensen \\
\hline & $\begin{array}{l}\text { Littlerock Post office } \\
\text { (USGS) }\end{array}$ & $\begin{array}{r}34.521 \mathrm{~N} \\
117.991 \mathrm{~W}\end{array}$ & (3) & 5.9 & $\begin{array}{l}300 \\
\text { Up } \\
210\end{array}$ & $\begin{array}{l}.08 \\
.07 \\
.07\end{array}$ & $\begin{array}{l}--- \\
--- \\
---\end{array}$ \\
\hline & $\begin{array}{l}\text { Lone Pine Canyon } \\
\text { (USGS) }\end{array}$ & $\begin{array}{r}34.32 \mathrm{~N} \\
117.57 \mathrm{~W}\end{array}$ & $42: 30.4$ & 6.4 & $\begin{array}{l}120 \\
\text { Up } \\
030\end{array}$ & $\begin{array}{l}.05 \\
.03 \\
.05\end{array}$ & $\begin{array}{l}--- \\
--- \\
---\end{array}$ \\
\hline & $\begin{array}{l}\text { Mal ibu Canyon } \\
\text { Monte Nido Fire Sta. } \\
\text { (USGS) }\end{array}$ & $\begin{array}{r}34.08 \mathrm{~N} \\
118.69 \mathrm{~W}\end{array}$ & (3) & (2) & & (1) & \\
\hline & $\begin{array}{l}\text { Palmdale Fire Station } \\
\text { (USGS) }\end{array}$ & $\begin{array}{r}34.58 \mathrm{~N} \\
118.11 \mathrm{~W}\end{array}$ & $42: 29.9$ & 6.8 & & (1) & \\
\hline & $\begin{array}{l}\text { Lytle Creek } \\
\text { Mann Residence } \\
\text { (USGS) }\end{array}$ & $\begin{array}{r}34.26 \mathrm{~N} \\
117.50 \mathrm{~W}\end{array}$ & $42: 39.7$ & (2) & $\begin{array}{l}315 \\
\text { Up } \\
225\end{array}$ & $\begin{array}{l}.04 \\
.02 \\
.05\end{array}$ & $\begin{array}{l}--- \\
--- \\
--\end{array}$ \\
\hline
\end{tabular}


Table 1. Catalogue of National Cooperative Strong-Motion Network accelerograph records recovered during 1987-Continued

\begin{tabular}{|c|c|c|c|c|c|c|c|}
\hline Earthquake & $\begin{array}{l}\text { Station name } \\
\text { (owner) }\end{array}$ & $\begin{array}{l}\text { Station } \\
\text { location } \\
\left({ }^{\circ}\right)\end{array}$ & $\begin{array}{l}\text { Trigger } \\
\text { time }\end{array}$ & $\begin{array}{l}\text { S-minus } \\
\text { trigger } \\
(\mathrm{s})\end{array}$ & $\begin{array}{l}\text { Direction } \\
\quad(a z)\end{array}$ & $\begin{array}{l}\text { Maximum } \\
\text { amp } 7 \text { i tude } \\
(\mathrm{g})\end{array}$ & $\begin{array}{l}\text { D.iration } \\
(\mathrm{s})\end{array}$ \\
\hline \multirow{9}{*}{$\begin{array}{l}1 \text { october } 1987 \\
1442: 20.0 \mathrm{G} . \mathrm{m} . \mathrm{t} . \\
\text { Southern Cal if. } \\
34.060 \mathrm{~N}, 118.080 \mathrm{~W} \\
\text { Magnitude } 5.9 \mathrm{ML}-- \\
\text { Continued }\end{array}$} & $\begin{array}{l}\text { Newport Beach } \\
840 \text { Newport Center Dr. } \\
\text { (USGS) }\end{array}$ & $\begin{array}{r}33.618 \mathrm{~N} \\
117.878 \mathrm{~W}\end{array}$ & (3) & 6.1 & & & \\
\hline & \multicolumn{4}{|c|}{$\begin{array}{l}\text { Structure Array: } \\
\text { Ch. 1- Tower 2, Leve1 } 1 \text { (Garage), Center } \\
\text { Ch. 2- Tower 2, Leve1 } 1 \text { (Garage), Center } \\
\text { Ch. 3- Tower 2, Level } 1 \text { (Garage), Center } \\
\text { Ch. 4- Tower 2, Level } 2 \text { (Plaza), West End } \\
\text { Ch. 5- Center Building, Level 2 (Plaza), Center } \\
\text { Ch. 6- Center Building, Level 2 (Plaza), Center } \\
\text { Ch. 7- Tower 2, Level } 9 \text { (Roof), South End } \\
\text { Ch. 8- Tower 2, Level } 10 \text { (Penthouse), Center } \\
\text { Ch. 9- Tower 2, Level } 10 \text { (Penthouse), Center } \\
\text { Ch. 10- Tower 1, Level } 9 \text { (Roof), East End } \\
\text { Ch. 11- Tower 1, Level } 10 \text { (Penthouse), Center } \\
\text { Ch. 12- Tower 1, Leve1 } 10 \text { (Penthouse), Center }\end{array}$} & $\begin{array}{l}360 \\
\text { Up } \\
090 \\
360 \\
360 \\
090 \\
090 \\
360 \\
090 \\
360 \\
270 \\
360\end{array}$ & $\begin{array}{l}.06 \\
.02 \\
.03 \\
.08 \\
.10 \\
.06 \\
.04 \\
.04 \\
.08 \\
.04 \\
.04 \\
.06\end{array}$ & $\begin{array}{l}--- \\
--- \\
--- \\
1 \text { peak } \\
--- \\
--- \\
--- \\
--- \\
--- \\
--- \\
---\end{array}$ \\
\hline & $\begin{array}{l}\text { Santa Ana River Bridge } \\
\text { (MWD/USGS) }\end{array}$ & $\begin{array}{r}33.968 \mathrm{~N} \\
117.447 \mathrm{~W}\end{array}$ & (3) & 2.6 & & & \\
\hline & \multicolumn{2}{|l|}{$\begin{array}{l}\text { North Abutment } \\
\text { Recorder Building }\end{array}$} & & & $\begin{array}{l}175 \\
\text { Up } \\
085\end{array}$ & $\begin{array}{l}.05 \\
.04 \\
.05\end{array}$ & $\begin{array}{l}--- \\
--- \\
---\end{array}$ \\
\hline & \multicolumn{2}{|c|}{$\begin{array}{l}\text { Structure Array: } \\
\text { Ch. 1- North Abutment } \\
\text { Ch. 2- North Abutment } \\
\text { Ch. 3- North Abutment } \\
\text { Ch. 4- Mid Span } \\
\text { Ch. 5- Mid Span } \\
\text { Ch. 6- Mid Span } \\
\text { Ch. 7- Below Bearing } \\
\text { Ch. 8- Below Bearing } \\
\text { Ch. 9- Below Bearing } \\
\text { Ch. 10- Above Bearing } \\
\text { Ch. 11- Above Bearing } \\
\text { Ch. 12- Above Bearing }\end{array}$} & & & $\begin{array}{l}346 \\
\text { Down } \\
076 \\
346 \\
\text { Down } \\
076 \\
346 \\
\text { Down } \\
076 \\
346 \\
\text { Down } \\
076\end{array}$ & $\begin{array}{l}.05 \\
.03 \\
.04 \\
.18 \\
.11 \\
.12 \\
.13 \\
.01 \\
.06 \\
.15 \\
.07 \\
.08\end{array}$ & $\begin{array}{ll}--- \\
--- \\
--- \\
2.3 \\
1 \\
2 \text { peak } \\
\text { peaks } \\
3 \text { peaks } \\
--- \\
--- \\
2.2 \\
--- \\
---\end{array}$ \\
\hline & $\begin{array}{l}\text { Mathews Dam } \\
\text { Dike Toe } \\
\text { (MWD) }\end{array}$ & $\begin{array}{r}33.852 \mathrm{~N} \\
117.451 \mathrm{~W}\end{array}$ & (3) & 6.2 & $\begin{array}{l}252 \\
\text { Up } \\
162\end{array}$ & $\begin{array}{l}.05 \\
.05 \\
.09\end{array}$ & $\begin{array}{l}--- \\
---\end{array}$ \\
\hline & $\begin{array}{l}\text { Leona Valley Fire Sta. } \\
\text { (USGS) }\end{array}$ & $\begin{array}{r}34.62 \mathrm{~N} \\
118.29 \mathrm{~W}\end{array}$ & (3) & 4.5 & $\begin{array}{l}120 \\
\text { Up } \\
030\end{array}$ & $\begin{array}{l}.05 \\
.04 \\
.03\end{array}$ & $\begin{array}{l}--- \\
---\end{array}$ \\
\hline & $\begin{array}{l}\text { Sunnymead } \\
\text { Egg Ranch } \\
\text { (USGS) }\end{array}$ & $\begin{array}{r}33.95 \mathrm{~N} \\
117.15 \mathrm{~W}\end{array}$ & $42: 35.7$ & 9.7 & & (1) & \\
\hline & $\begin{array}{l}\text { Malibu } \\
\text { Kilpatrick School } \\
\text { (USGS) }\end{array}$ & $\begin{array}{r}34.093 \mathrm{~N} \\
118.836 \mathrm{~W}\end{array}$ & $42: 43.1$ & (2) & & (1) & \\
\hline
\end{tabular}


Table 1. Catalogue of National Cooperative Strong-Motion Network accelerograph records recoveren during 1987-Continued

\begin{tabular}{|c|c|c|c|c|c|c|c|}
\hline Earthquake & $\begin{array}{l}\text { Station name } \\
\text { (owner) }\end{array}$ & $\begin{array}{l}\text { Station } \\
\text { location } \\
\left({ }^{\circ}\right)\end{array}$ & $\begin{array}{l}\text { Trigger } \\
\text { time }\end{array}$ & $\begin{array}{c}\text { S-minus } \\
\text { trigger } \\
\text { (s) }\end{array}$ & $\begin{array}{l}\text { Direction } \\
\quad(a z)\end{array}$ & $\begin{array}{l}\text { Max imum } \\
\text { amplitude. } \\
\text { (g) }\end{array}$ & $\begin{array}{c}\text { Duration } \\
\text { (s) }\end{array}$ \\
\hline \multirow{7}{*}{$\begin{array}{l}1 \text { 0ctober } 1987 \\
1442: 20.0 \mathrm{G.m.t.} \\
\text { Southern Calif. } \\
34.060 \mathrm{~N}, 118.080 \mathrm{~W} \\
\text { Magnitude } 5.9 \mathrm{ML}-- \\
\text { Continued }\end{array}$} & $\begin{array}{l}\text { Santa Susana } \\
\text { (DOE) }\end{array}$ & $\begin{array}{r}34.23 \mathrm{~N} \\
118.71 \mathrm{~W}\end{array}$ & (3) & (2) & & & \\
\hline & Ground Station & & & & & (1) & \\
\hline & Building 026, Ground & & & & & (1) & \\
\hline & $\begin{array}{c}\text { Building } 356, \\
\text { 3rd Floor }\end{array}$ & & & & $\begin{array}{l}325 \\
\text { Up } \\
235\end{array}$ & $\begin{array}{l}.07 \\
.03 \\
.07\end{array}$ & $\begin{array}{l}--- \\
---\end{array}$ \\
\hline & $\begin{array}{l}\text { Building 462: } \\
\text { 1st Floor }\end{array}$ & & & & & (1) & \\
\hline & 6th Floor & & & & $\begin{array}{l}090 \\
\text { Up } \\
360\end{array}$ & $\begin{array}{l}.08 \\
.04 \\
.07\end{array}$ & $\begin{array}{l}--- \\
---\end{array}$ \\
\hline & Building 463, Roof & & & & $\begin{array}{l}090 \\
\text { Up } \\
360\end{array}$ & $\begin{array}{l}.07 \\
.09 \\
.07\end{array}$ & $\begin{array}{l}--- \\
---\end{array}$ \\
\hline
\end{tabular}

$\begin{array}{lrll}\text { Colton Interchange } & 34.06 \mathrm{~N} & \text { (3) } & \text { (2) } \\ \text { I-10/I-215 } & 117.30 \mathrm{~W} & & \\ \text { Pier Base Vault (CDOT) } & & & \\ \begin{array}{l}\text { San Bernardino } \\ \text { County Government Ctr. } 117.287 \mathrm{~W} \\ \text { (USGS) }\end{array} & 42: 36.6 & 5.2 \\ \end{array}$

Ground Floor, SW

Structure Array:
Ch. 1- 2nd Floor Level, NW
Ch. 2- 2nd Floor Level, NE
Ch. 3- 2nd Floor Level, NE
Ch. 4- 2nd Floor Level, SW
Ch. 5- 4th Floor Level, SW
Ch. 6- 4th Floor Level, NW
Ch. 7- 6th Floor Level, (Roof) NE
Ch. 8- 6th Floor Level, (Roof) NW
Ch. 9- 6th Floor Level, (Roof) SW
Ch. 10- Sensor Not Installed
Ch. 11- 4th Floor Level, NE
Ch. 12- 4 th Floor Level, NE

Reche Canyon

01 ive Del1 Ranch

(USGS)

Loma Linda Med. Ctr. Basement

(USGS) $\begin{array}{llll}34.01 & N & 42: 44.8 & \text { (2) }\end{array}$

$117.22 \mathrm{~W}$

$34.05 \mathrm{~N}$

$117.26 \mathrm{~W}$

(3)

(2)
---
---
---
---
---
---
---
1.4
---
$(---)$
---
---

(1) 
Table 1. Catalogue of National Cooperative Strong-Motion Network accelerograph records recovered during 1987-Continued

\begin{tabular}{|c|c|c|c|c|c|c|c|}
\hline Earthquake & $\begin{array}{l}\text { Station name } \\
\text { (owner) }\end{array}$ & $\begin{array}{l}\text { Station } \\
\text { location } \\
\left({ }^{\circ}\right)\end{array}$ & $\begin{array}{c}\text { Trigger } \\
\text { time }\end{array}$ & $\begin{array}{l}\text { S-minus } \\
\text { trigger } \\
(\mathrm{s})\end{array}$ & $\begin{array}{l}\text { Direction } \\
\quad(a z)\end{array}$ & $\begin{array}{l}\text { Maximum } \\
\text { amp } 1 \text { itude } \\
(\mathrm{g})\end{array}$ & $\begin{array}{l}\text { Duration } \\
\text { (s) }\end{array}$ \\
\hline \multirow{4}{*}{$\begin{array}{l}1 \text { 0ctober } 1987 \\
1442: 20.0 \text { G.m.t. } \\
\text { Southern Calif. } \\
34.060 \mathrm{~N}, 118.080 \mathrm{~W} \\
\text { Magnitude } 5.9 \mathrm{ML}-- \\
\text { Continued }\end{array}$} & $\begin{array}{l}\text { Loma Linda } \\
\text { VA Hospital } \\
\text { (VA/USGS) }\end{array}$ & $\begin{array}{r}34.049 \mathrm{~N} \\
117.250 \mathrm{~W}\end{array}$ & (3) & 7.8 & & & \\
\hline & $\begin{array}{l}\text { Building } 1 \text { Structur } \\
\text { Ch. 1- Ground Flo } \\
\text { Ch. 2- Ground Flo } \\
\text { Ch. 3- Ground Flo } \\
\text { Ch. 4- 4th Floor, } \\
\text { Ch. 5- Ground Flo } \\
\text { Ch. 6- 4th Floor, } \\
\text { Ch. 7- 4th Floor, } \\
\text { Ch. 8- Ground Flo } \\
\text { Ch. 9- 4th Floor, }\end{array}$ & $\begin{array}{l}\text { e Array: } \\
\text { or, Center } \\
\text { or, Center } \\
\text { or, Center } \\
\text { Center } \\
\text { or, North } \\
\text { Center } \\
\text { North } \\
\text { or, South } \\
\text { South }\end{array}$ & & & $\begin{array}{l}\text { Down } \\
180 \\
270 \\
270 \\
270 \\
180 \\
270 \\
180 \\
270\end{array}$ & $\begin{array}{l}.01 \\
.03 \\
.02 \\
.07 \\
.03 \\
.04 \\
.08 \\
.01 \\
.07\end{array}$ & $\begin{array}{l}--- \\
--- \\
--- \\
--- \\
--- \\
--- \\
--- \\
--- \\
---\end{array}$ \\
\hline & North Ground Site & & $42: 35.2$ & (2) & & (1) & \\
\hline & South Ground Site & & (3) & (2) & & (1) & \\
\hline \multirow{10}{*}{$\begin{array}{l}\text { 1 October } 1987 \\
\text { 1445:41.4 G.m.t. } \\
\text { Southern Calif. } \\
34.050 \mathrm{~N}, 118.100 \mathrm{~W} \\
\text { Magnitude } 4.6 \mathrm{ML}\end{array}$} & $\begin{array}{l}\text { Norwalk, } 12440 \\
\text { Imperial Highway } \\
\text { (USGS/BECH) }\end{array}$ & $\begin{array}{r}32.92 \mathrm{~N} \\
118.07 \mathrm{~W}\end{array}$ & $45: 50.8$ & (2) & & & \\
\hline & Basement & & & & & (1) & \\
\hline & North Freefield & & & & & (1) & \\
\hline & South Freefield & & & & & (1) & \\
\hline & Structure Arrays 1 & and 2 & & & & (1) & \\
\hline & $\begin{array}{l}\text { Los Angeles } \\
1100 \text { Wilshire Blvd. } \\
\text { (JCG/USGS) }\end{array}$ & $\begin{array}{r}34.052 \mathrm{~N} \\
118.263 \mathrm{~W}\end{array}$ & $45: 49.9$ & (2) & & & \\
\hline & Basement 3 , NE & & & & & (1) & \\
\hline & Basement 3, SE & & & & & (1) & \\
\hline & Basement 4 , NW & & & & & (1) & \\
\hline & Structure Array & & & & & (1) & \\
\hline \multirow{4}{*}{$\begin{array}{l}1 \text { 0ctober } 1987 \\
\text { 1449:05.9 G.m.t. } \\
\text { Southern Calif. } \\
\text { 34.060N, 118.100W } \\
\text { Magnitude 4.7 ML }\end{array}$} & $\begin{array}{l}\text { Norwalk, } 12440 \\
\text { Imperial Highway } \\
\text { (USGS/BECH) }\end{array}$ & $\begin{array}{r}32.92 \mathrm{~N} \\
118.07 \mathrm{~W}\end{array}$ & $49: 14.4$ & (2) & & & \\
\hline & Basement & & & & & (1) & \\
\hline & North Freefield & & & & & (1) & \\
\hline & South Freefield & & & & & (1) & \\
\hline
\end{tabular}


Table 1. Catalogue of National Cooperative Strong-Motion Network accelerograph records recove"ed during 1987-Continued

\begin{tabular}{llllll}
\hline Earthquake & $\begin{array}{c}\text { Station name } \\
\text { (owner) }\end{array}$ & $\begin{array}{c}\text { Station } \\
\text { location } \\
\left({ }^{\circ}\right)\end{array}$ & $\begin{array}{c}\text { Trigger } \\
\text { time }\end{array}$ & $\begin{array}{c}\text { s-minus Direction Maximum } \\
\text { trigger } \\
(\mathrm{s})\end{array}$ & $\begin{array}{c}\text { Duration } \\
\text { amplitude } \\
(\mathrm{g})\end{array}$ \\
\hline
\end{tabular}

1 October $1987 \quad$ Norwalk, 12440--Cont inued

1449:05.9 G.m.t. Structure Arrays 1 and 2

Southern Calif.

$34.060 \mathrm{~N}, 118.100 \mathrm{~W}$ Los Angeles

$\begin{array}{lr}34.060 \mathrm{~N}, 118.100 \mathrm{~W} \text { LOS Angeles } & 34.052 \mathrm{~N} \\ \text { Magnitude 4.7 ML-- } 1100 \text { Wilshire Blyd. } & 118.263 \mathrm{~W}\end{array}$

Cont inued

(JCG/USGS)

Basement 3, NE

Basement 3, SE

Basement 4, NW

Structure Array

1 October $1987 \quad$ LOS Angeles

$34.052 N$

1512:31.7 G.m.t.

Southern Cal if.

$34.050 \mathrm{~N}, 118.090 \mathrm{~W}$

Magnitude $4.7 \mathrm{ML}$

(JCG/USGS)

Basement 3, NE

Basement 3, SE

Basement 4, NW

Structure Array

1 0ctober 1987

1559:53.5 G.m.t.

Southern Calif.

$34.050 \mathrm{~N}, 118.090 \mathrm{~W}$

Magnitude $4.0 \mathrm{ML}$

Los Ange les

$34.052 \mathrm{~N}$

1100 Wilshire Blvd. $\quad 118.263 \mathrm{~N}$

$00: 01.7$

(2) (JCG/USGS)

Basement 3, NE

Basement 3, SE

Basement 4, NW

Structure Array

1 0ctober 1987

Whittier

$33.977 \mathrm{~N}$

1442-1957 G.m.t.

Southern Calif.

Epicenters and

7215 Bright Avenue

$118.036 \mathrm{~W}$

(3)

2.5

magnitudes unknown

Basement

$\begin{array}{lll}180 & .09 & --- \\ \text { Up } & .04 & --- \\ 090 & .09 & --- \\ 180 & .08 & --- \\ \text { Up } & .04 & --- \\ 090 & .08 & --- \\ 180 & .08 & --- \\ \text { Up } & .05 & --- \\ 090 & .10 & 1 \text { peak }\end{array}$


Table 1. Catalogue of National Cooperative Strong-Motion Network accelerograph records recovered during 1987-Continued

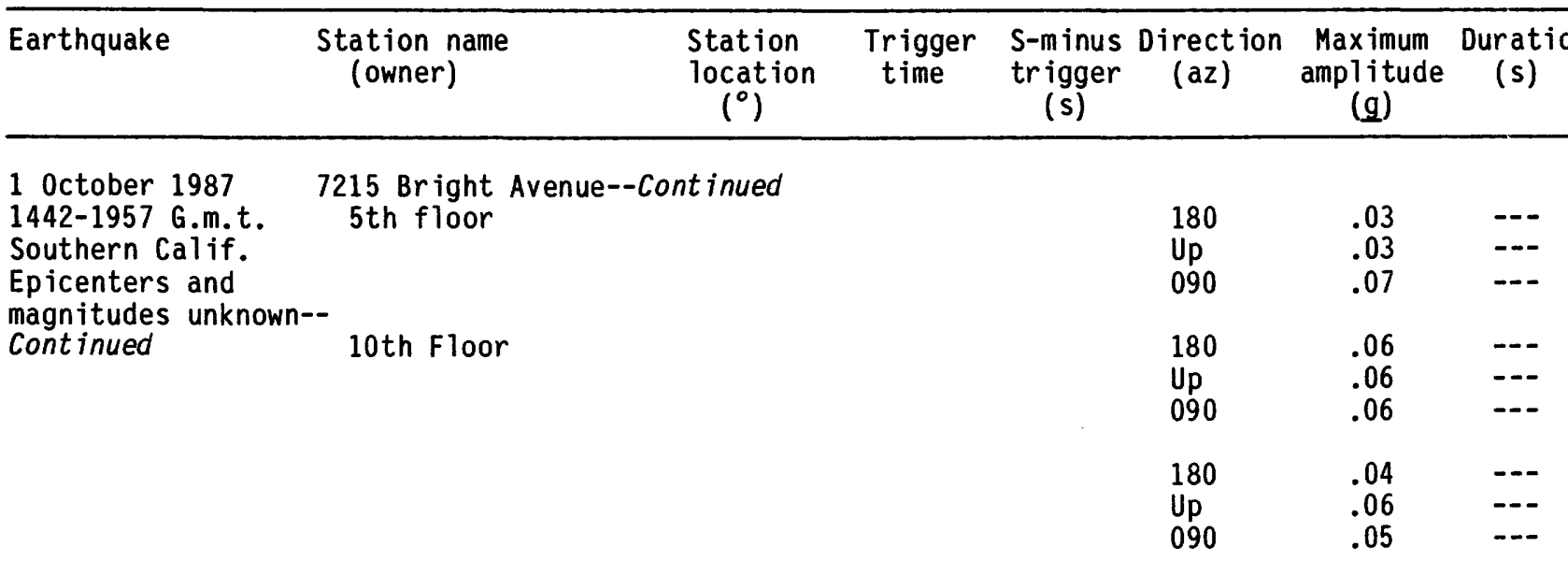

Note: Three additional records ${ }^{1}$ in basement, one from 5 th floor, and two from 10 th floor recovered at Whittier.

1 0ctober 1987 Whittier Narrows Dam $34.03 \mathrm{~N}$

1442-2012 G.m.t. (ACOE)

Southern Calif.

Epicenters and

magnitudes unknown
Crest

Upstream

\section{$118.05 \mathrm{~W}$}

(3)

\section{1}

$\begin{array}{lcc}033 & .10 & 1 \text { peak } \\ \text { Up } & .04 & -.- \\ 303 & .09 & --- \\ 033 & .13 & 0.2 \\ \text { Up } & .05 & -.- \\ 303 & .12 & 0.4 \\ 152 & .09 & --- \\ \text { Up } & .07 & --- \\ 062 & .13 & 0.2 \\ 152 & .07 & --- \\ \text { Up } & .04 & -.- \\ 062 & .09 & --- \\ 152 & .12 & 1 \text { peak } \\ \text { Up } & .07 & --- \\ 062 & .10 & 1 \text { peak }\end{array}$

Note: Two additional records ${ }^{1}$ from crest and four records from upstream recovered at Whittier Narrows Dam.

1 0ctober 19872 0ctober 1987 Southern Calif. Epicenters and magnitudes unknown

$$
\begin{array}{lr}
\text { Garvey Reservoir } & 34.05 \mathrm{~N} \\
\text { Abutment Building } & 118.11 \mathrm{~W}
\end{array}
$$
(MWD)
(3)

2.0

060
Up
330

.12

.06

.10

2.0

060

330

$\begin{array}{ll}2.0 & 060 \\ & \text { Up } \\ & 330\end{array}$

.15

.06

.09

.11

.08

.07
1 peak

0.2

$-$

1 peak

Note: Seven additional records ${ }^{1}$ recovered at Garvey Reservoir. 
Table 1. Catalogue of National Cooperative Strong-Motion Network accelerograph records recover $\geqq d$ during 1987-Continued

\begin{tabular}{|c|c|c|c|c|c|c|c|}
\hline Earthquake & $\begin{array}{l}\text { Station name } \\
\text { (owner) }\end{array}$ & $\begin{array}{l}\text { Station } \\
\text { location } \\
\left({ }^{\circ}\right)\end{array}$ & $\begin{array}{l}\text { Trigger } \\
\text { time }\end{array}$ & $\begin{array}{l}\text { S-minus } \\
\text { trigger } \\
(\mathbf{s})\end{array}$ & $\begin{array}{l}\text { Direction } \\
\quad(a z)\end{array}$ & $\begin{array}{l}\text { Maximum } \\
\text { amplitude } \\
(\mathrm{g})\end{array}$ & $\begin{array}{l}\text { Duration } \\
\text { (s) }\end{array}$ \\
\hline \multirow{3}{*}{$\begin{array}{l}1 \text { October } 1987- \\
3 \text { October } 1987 \\
\text { Southern Cal if. } \\
\text { Epicenters and } \\
\text { magnitudes unknown }\end{array}$} & $\begin{array}{l}\text { Los Angeles } \\
\text { Bulk Mail Facility } \\
\text { (USGS) }\end{array}$ & $\begin{array}{r}33.996 \mathrm{~N} \\
118.162 \mathrm{~W}\end{array}$ & (3) & 2.6 & $\begin{array}{l}010 \\
\text { Up } \\
280\end{array}$ & $\begin{array}{l}.06 \\
.02 \\
.06\end{array}$ & 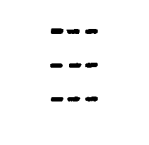 \\
\hline & & & (3) & 2.6 & $\begin{array}{l}010 \\
\text { Up } \\
280\end{array}$ & $\begin{array}{l}.12 \\
.04 \\
.16\end{array}$ & $\begin{array}{l}1 \text { peak } \\
0.4\end{array}$ \\
\hline & & & (3) & 2.6 & $\begin{array}{l}010 \\
\text { Up } \\
280\end{array}$ & $\begin{array}{l}.06 \\
.05 \\
.07\end{array}$ & $\begin{array}{l}--- \\
--- \\
---\end{array}$ \\
\hline
\end{tabular}

Note: 7 additional records ${ }^{1}$ recovered at Los Angeles Bulk Mail Facility.

\begin{tabular}{|c|c|c|c|c|c|c|c|}
\hline $\begin{array}{l}1 \text { October } 1987- \\
4 \text { October 1987 } \\
\text { Southern Calif. } \\
\text { Epicenters and } \\
\text { magnitudes unknown }\end{array}$ & $\begin{array}{l}\text { Vernon } \\
4814 \text { Loma Vista Ave. } \\
\text { (USGS) } \\
\quad \text { Note: One additic }\end{array}$ & $\begin{array}{r}34.00 \mathrm{~N} \\
118.20 \mathrm{~W}\end{array}$ & recove & at Ver & $\begin{array}{l}007 \\
\text { Up } \\
277\end{array}$ & $\begin{array}{l}.08 \\
.02 \\
.11\end{array}$ & $\begin{array}{l}--. \\
--. \\
0.2\end{array}$ \\
\hline \multirow{8}{*}{$\begin{array}{l}4 \text { october } 1987 \\
\text { 1059:38.1 G.m.t. } \\
\text { Southern Calif. } \\
\text { 34.070N, 118.100W } \\
\text { Magnitude } 5.3 \mathrm{ML}\end{array}$} & $\begin{array}{l}\text { Garvey Reservoir } \\
\text { Abutment Bldg. } \\
\text { (MWD) }\end{array}$ & $\begin{array}{r}34.05 \mathrm{~N} \\
118.11 \mathrm{~W}\end{array}$ & (3) & 1.9 & $\begin{array}{l}060 \\
\text { Up } \\
330\end{array}$ & $\begin{array}{l}.19 \\
.13 \\
.21\end{array}$ & $\begin{array}{l}0.3 \\
1.6 \\
0.7\end{array}$ \\
\hline & $\begin{array}{l}\text { Alhambra } \\
900 \mathrm{~S} \text {. Fremont Ave. } \\
\text { (USGS) }\end{array}$ & $\begin{array}{r}34.09 \mathrm{~N} \\
118.15 \mathrm{~W}\end{array}$ & (3) & 1.9 & & & \\
\hline & Basement & & & & $\begin{array}{l}090 \\
\text { Up } \\
360\end{array}$ & $\begin{array}{l}.15 \\
.10 \\
.11\end{array}$ & $\begin{array}{l}0.3 \\
1 \text { peak } \\
2 \text { peaks }\end{array}$ \\
\hline & 6th Floor & & & & $\begin{array}{l}090 \\
\text { Up } \\
360\end{array}$ & $\begin{array}{l}.24 \\
.15 \\
.17\end{array}$ & $\begin{array}{l}0.4 \\
3 \text { peaks } \\
0.8\end{array}$ \\
\hline & 12th Floor & & & & $\begin{array}{l}090 \\
\text { Up } \\
360\end{array}$ & $\begin{array}{l}.18 \\
.19 \\
.13\end{array}$ & $\begin{array}{l}0.5 \\
2.7 \\
0.8\end{array}$ \\
\hline & $\begin{array}{l}\text { Whittier Narrows Dam } \\
(A C O E)\end{array}$ & $\begin{array}{r}34.03 \mathrm{~N} \\
118.05 \mathrm{~W}\end{array}$ & (3) & 1.9 & & & \\
\hline & Crest & & & & $\begin{array}{l}033 \\
\text { Up } \\
303\end{array}$ & $\begin{array}{l}.21 \\
.09 \\
.25\end{array}$ & $\begin{array}{l}0.9 \\
--\overline{1.1}\end{array}$ \\
\hline & $\begin{array}{l}\text { Los Angeles } \\
4407 \text { Jasper Street } \\
\text { (USGS) }\end{array}$ & $\begin{array}{r}34.081 \mathrm{~N} \\
118.188 \mathrm{~W}\end{array}$ & (3) & 1.8 & $\begin{array}{l}130 \\
\text { Up } \\
040\end{array}$ & $\begin{array}{l}.17 \\
.10 \\
.17\end{array}$ & $\begin{array}{l}1.6 \\
1 \text { peak } \\
0.5\end{array}$ \\
\hline
\end{tabular}


Table 1. Catalogue of National Cooperative Strong-Motion Network accelerograph records recovered d:uring 1987-Continued

\begin{tabular}{|c|c|c|c|c|c|c|c|}
\hline Earthquake & $\begin{array}{l}\text { Station name } \\
\text { (owner) }\end{array}$ & $\begin{array}{l}\text { Station } \\
\text { location } \\
\left({ }^{\circ}\right)\end{array}$ & $\begin{array}{l}\text { Trigger } \\
\text { time }\end{array}$ & $\begin{array}{l}\text { S-minus } \\
\text { trigger } \\
(\mathrm{s})\end{array}$ & $\begin{array}{l}\text { Direction } \\
\quad(a z)\end{array}$ & $\begin{array}{l}\text { Maximum } \\
\text { amplitude } \\
\text { (g) }\end{array}$ & $\begin{array}{l}\text { Duration } \\
\text { (s) }\end{array}$ \\
\hline \multirow[t]{13}{*}{$\begin{array}{l}4 \text { october } 1987 \\
\text { 1059:38.1 G.m.t. } \\
\text { Southern Calif. } \\
\text { 34.070N, 118.100W } \\
\text { Magnitude 5.3 ML-- } \\
\text { Continued }\end{array}$} & $\begin{array}{l}\text { Los Angeles } \\
\text { Bulk Mail Center } \\
\text { (USGS) } \\
\text { Whittier } \\
7215 \text { Bright Ave. } \\
\text { USGS) }\end{array}$ & $\begin{array}{r}33.99 \mathrm{~N} \\
118.16 \mathrm{~W} \\
\\
33.977 \mathrm{~N} \\
118.036 \mathrm{~W}\end{array}$ & (3) & 2.3 & $\begin{array}{l}010 \\
\text { Up } \\
280\end{array}$ & $\begin{array}{l}.24 \\
.08 \\
.25\end{array}$ & \begin{tabular}{l}
$c .5$ \\
\hdashline.-
\end{tabular} \\
\hline & Basement & & & & $\begin{array}{l}180 \\
\text { Up } \\
090\end{array}$ & $\begin{array}{l}.30 \\
.15 \\
.33\end{array}$ & $\begin{array}{l}C .8 \\
C .7 \\
C .9\end{array}$ \\
\hline & 5 th Floor & & & & $\begin{array}{l}180 \\
\text { Up } \\
090\end{array}$ & $\begin{array}{l}.30 \\
.28 \\
.58\end{array}$ & $\begin{array}{l}C .7 \\
3.3 \\
3.4\end{array}$ \\
\hline & Letion & & & & $\begin{array}{l}180 \\
\text { Up } \\
090\end{array}$ & $\begin{array}{l}.17 \\
.53 \\
.43\end{array}$ & $\begin{array}{l}C .5 \\
3.4 \\
4.3\end{array}$ \\
\hline & $\begin{array}{l}\text { Vernon } \\
4814 \text { Loma Vișta Ave. } \\
\text { (USGS) }\end{array}$ & $\begin{array}{r}34.00 \mathrm{~N} \\
118.20 \mathrm{~W}\end{array}$ & (3) & 2.5 & $\begin{array}{l}007 \\
U p \\
277\end{array}$ & $\begin{array}{l}.15 \\
.07 \\
.27\end{array}$ & \begin{tabular}{l}
$C .5$ \\
\hdashline. $\bar{C}$
\end{tabular} \\
\hline & $\begin{array}{l}\text { Los Angeles } \\
1111 \text { Sunset Blvd. } \\
\text { (MWD) }\end{array}$ & $\begin{array}{r}34.07 \mathrm{~N} \\
118.25 \mathrm{~W}\end{array}$ & (3) & 2.4 & & & \\
\hline & Basement & & & & $\begin{array}{l}348 \\
\text { Up } \\
258\end{array}$ & $\begin{array}{l}.08 \\
.05 \\
.08\end{array}$ & $\begin{array}{l}-- \\
-- \\
--\end{array}$ \\
\hline & 4th Floor & & & & $\begin{array}{l}348 \\
\text { Up } \\
258\end{array}$ & $\begin{array}{l}.11 \\
.07 \\
.10\end{array}$ & $\begin{array}{l}1 \text { peak } \\
1 \text { peak }\end{array}$ \\
\hline & Roof & & & & $\begin{array}{l}348 \\
\text { Up } \\
258\end{array}$ & $\begin{array}{l}.10 \\
.10 \\
.08\end{array}$ & $\begin{array}{l}1 \text { peak } \\
1 \text { peak } \\
---\end{array}$ \\
\hline & $\begin{array}{l}\text { Los Angeles } \\
\text { 1100 Wilshire Bivd. } \\
\text { (JCG/USGS) }\end{array}$ & $\begin{array}{r}34.052 \mathrm{~N} \\
118.263 \mathrm{~W}\end{array}$ & $59: 41.9$ & 2.9 & & & \\
\hline & Basement 3 , NE & & & & $\begin{array}{l}298 \\
\text { Up } \\
208\end{array}$ & $\begin{array}{l}.07 \\
.04 \\
.09\end{array}$ & $\begin{array}{l}--- \\
--- \\
--\end{array}$ \\
\hline & Basement 3, SE & & & & $\begin{array}{l}298 \\
\text { Up } \\
208\end{array}$ & $\begin{array}{l}.08 \\
.04 \\
.08\end{array}$ & $\begin{array}{l}--- \\
--- \\
--\end{array}$ \\
\hline & Ba sement $4, \mathrm{NW}$ & & & & $\begin{array}{l}298 \\
\text { Up } \\
208\end{array}$ & $\begin{array}{l}.06 \\
.05 \\
.07\end{array}$ & $\begin{array}{l}-- \\
-- \\
--\end{array}$ \\
\hline
\end{tabular}


Table 1. Catalogue of National Cooperative Strong-Motion Network accelerograph records recovered during 1987-Continued

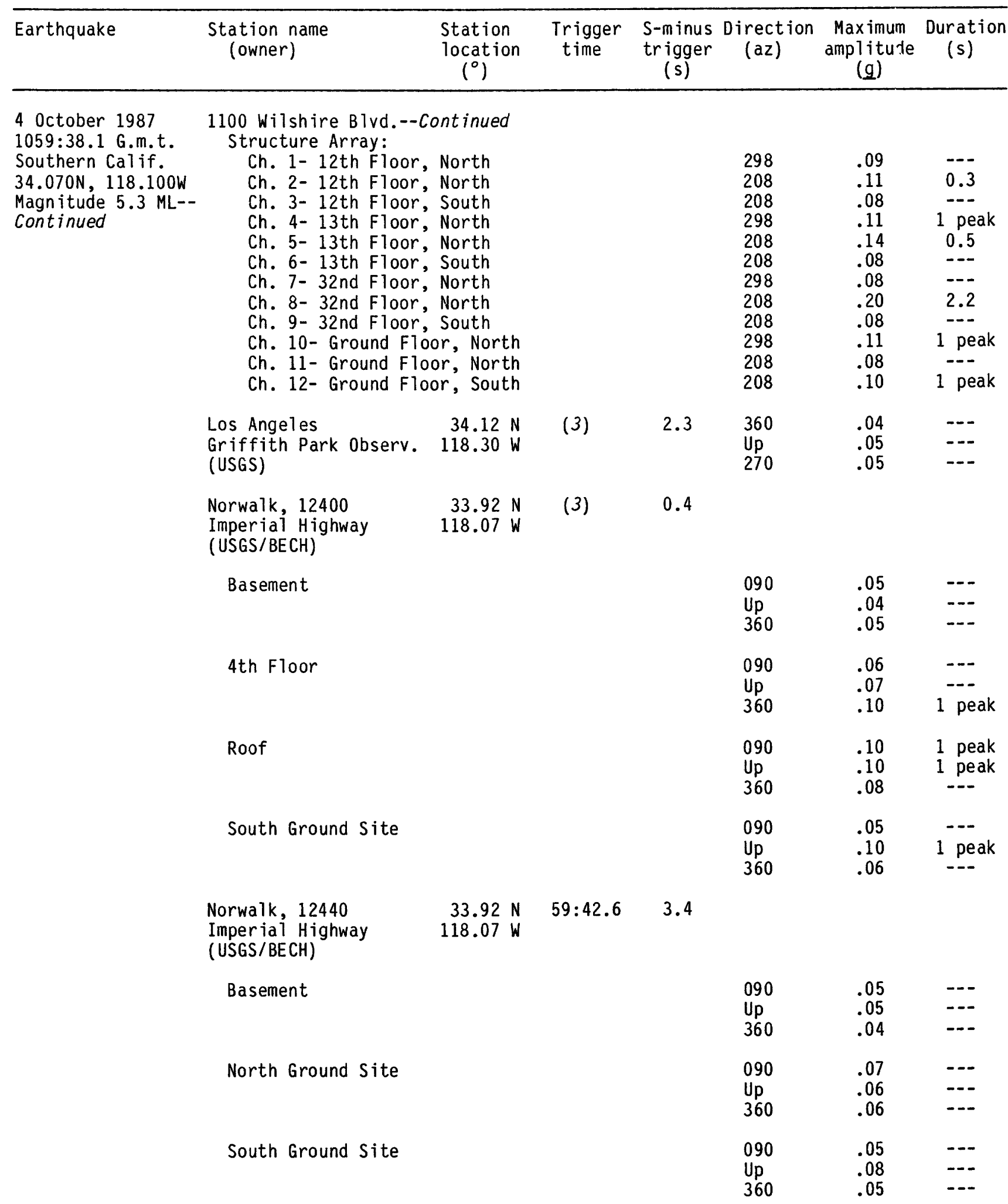


Table 1. Catalogue of National Cooperative Strong-Motion Network accelerograph records recovered during 1987-Continued

\begin{tabular}{|c|c|c|c|c|c|c|c|}
\hline Earthquake & $\begin{array}{l}\text { Station name } \\
\text { (owner) }\end{array}$ & $\begin{array}{c}\text { Station } \\
\text { location } \\
\left({ }^{\circ}\right)\end{array}$ & $\begin{array}{l}\text { Trigger } \\
\text { time }\end{array}$ & $\begin{array}{c}\text { S-minus } \\
\text { trigger } \\
(\mathrm{s})\end{array}$ & $\begin{array}{l}\text { Direction } \\
\quad(a z)\end{array}$ & $\begin{array}{l}\text { Maximum } \\
\text { ampl itude } \\
\text { (g) }\end{array}$ & $\begin{array}{c}\text { Duration } \\
\text { (s) }\end{array}$ \\
\hline
\end{tabular}

4 October $1987 \quad$ Norwalk, 12440--Continued

1059:38.1 G.m.t. Structure Array 1:

Southern Calif. $34.070 \mathrm{~N}, 118.100 \mathrm{~W}$ Magnitude 5.3 ML-Cont inued

Ch. 1-8th Floor (Roof), Center

Ch. 2- 5th Floor, Center

Ch. 3- 2nd Floor, Center

Ch. 4- 1st Floor, Center

Ch. 5- Basement, East

Ch. 6- 5th Floor, West-Center

Ch. 7- Basement, Center

Ch. 8- Basement, Center

Ch. 9- Basement, Center

Ch. 10- $30 \mathrm{ft}$. Downhole, B $1 \mathrm{dg}$. Center

Ch. 11- $30 \mathrm{ft}$. Downhole, B ldg. Center

Ch. 12- $30 \mathrm{ft}$. Downhole, Bldg. Center

Structure Array 2:

Ch. 13- 8th Floor (Roof), East

Ch. 14- 5th Floor, East

Ch. 15- 2nd Floor, East

Ch. 16- 1st Floor, East

Ch. 17- 8th Floor (Roof), Center

Ch. 18- 5th Floor, Center

Ch. 19- 2nd Floor, Center

Ch. 20- 1st Floor, Center

Ch. 21- 8th Floor (Roof), West

Ch. 22- 5th Floor, West

Ch. 23- 2nd Floor, West

Ch. 24- 1st Floor, West

Orange County Rsvr. $\quad 33.935 \mathrm{~N}$

Abutment

$117.883 \mathrm{~W}$

(MWD)

(3)

Long Beach, CSULB

Humanities B 1dg. Bsmt. 118.112W (USGS)

Lawndale (USGS)

Brea Dam

(ACOE)

$117.926 \mathrm{~W}$

Crest

Left Abutment

Downstream
$33.777 \mathrm{~N}$

$33.889 \mathrm{~N}$

$118.377 \mathrm{~W}$

(3)

(3)

(3)

3.2

4.1 $\begin{array}{ll}5.3 & 104 \\ & \text { Up } \\ & 076\end{array}$

090

090

090

360

360

Up

090

360

Up

090

360

360

360

360

360

360

360

360

360

360

360

360

360

$3.6 \quad 096$

Up

006

(1)
.03

.04

$\begin{array}{ll}.09 & --- \\ .07 & --- \\ .05 & --- \\ .06 & --- \\ .05 & --- \\ .07 & --- \\ .03 & --- \\ .05 & --- \\ .05 & --- \\ .03 & --- \\ .04 & --- \\ .03 & ---\end{array}$

.08

.04

.05

.08

INOP

.05

.07

.09

.05

.04

.03

.06

.04

.07

.06

03
.04

-.-

$--$

$\begin{array}{lc}\text { Crest } & 130 \\ & \text { Up } \\ & 040 \\ \text { Left Abutment } & 130 \\ & \text { Up } \\ & 040 \\ \text { Downstream } & 130 \\ & \text { Up } \\ & 040\end{array}$


Table 1. Catalogue of National Cooperative Strong-Motion Network accelerograph records recovered during 1987-Continued

\begin{tabular}{|c|c|c|c|c|c|c|c|}
\hline Earthquake & $\begin{array}{l}\text { Station name } \\
\text { (owner) }\end{array}$ & $\begin{array}{l}\text { Station } \\
\text { location } \\
\left({ }^{\circ}\right)\end{array}$ & $\begin{array}{l}\text { Trigger } \\
\text { time }\end{array}$ & $\begin{array}{l}\text { S-minus } \\
\text { trigger } \\
(\mathrm{s})\end{array}$ & $\begin{array}{l}\text { Direction } \\
\quad(a z)\end{array}$ & $\begin{array}{l}\text { Maximum } \\
\text { amplitude } \\
(\mathrm{g})\end{array}$ & $\begin{array}{l}\text { Duration } \\
\text { (s) }\end{array}$ \\
\hline \multirow{15}{*}{$\begin{array}{l}4 \text { 0ctober } 1987 \\
1059: 38.1 \text { G.m.t. } \\
\text { Southern Calif. } \\
34.070 \mathrm{~N}, 118.100 \mathrm{~W} \\
\text { Magnitude } 5.3 \mathrm{ML}-- \\
\text { Continued }\end{array}$} & $\begin{array}{l}\text { Carbon Canyon Dam } \\
\text { (ACOE) }\end{array}$ & $\begin{array}{r}33.92 \mathrm{~N} \\
117.84 \mathrm{~W}\end{array}$ & (3) & 4.2 & & & \\
\hline & Crest & & & & $\begin{array}{l}130 \\
\text { Up } \\
040\end{array}$ & $\begin{array}{l}.06 \\
.04 \\
.07\end{array}$ & $\begin{array}{l}--- \\
--- \\
---\end{array}$ \\
\hline & Left Abutment & & & & $\begin{array}{l}130 \\
\text { Up } \\
040\end{array}$ & $\begin{array}{l}.05 \\
.03 \\
.05\end{array}$ & $\begin{array}{l}--- \\
--- \\
--\end{array}$ \\
\hline & Right Abutment & & & & $\begin{array}{l}130 \\
\text { Up } \\
040\end{array}$ & $\begin{array}{l}.07 \\
.03 \\
.05\end{array}$ & $\begin{array}{l}-- \\
-- \\
--\end{array}$ \\
\hline & $\begin{array}{l}\text { Weymouth Filter Plant } \\
\text { (MWD) }\end{array}$ & $\begin{array}{r}34.114 \mathrm{~N} \\
117.778 \mathrm{~W}\end{array}$ & (3) & (2) & & & \\
\hline & Bldg., Ground Level & & & & & (1) & \\
\hline & Water Tank, Top & & & & $\begin{array}{l}017 \\
\text { Up } \\
287\end{array}$ & $\begin{array}{l}.05 \\
.04 \\
.07\end{array}$ & $\begin{array}{l}--- \\
--- \\
--\end{array}$ \\
\hline & $\begin{array}{l}\text { Diemer Filter Plant } \\
\text { (MWD) }\end{array}$ & $\begin{array}{r}33.91 \mathrm{~N} \\
117.82 \mathrm{~W}\end{array}$ & (3) & 4.3 & & & \\
\hline & Admin. Bldg. Bsmt. & & & & & $(1)$ & \\
\hline & Reservoir Roof & & & & $\begin{array}{l}281 \\
\text { Up } \\
191\end{array}$ & $\begin{array}{l}.04 \\
.05 \\
.06\end{array}$ & $\begin{array}{l}--- \\
---\end{array}$ \\
\hline & $\begin{array}{l}\text { Sepulveda VA Hospital } \\
\text { Ground level } \\
\text { (VA) }\end{array}$ & $\begin{array}{r}34.249 \mathrm{~N} \\
118.475 \mathrm{~W}\end{array}$ & (3) & 5.4 & $\begin{array}{l}360 \\
\text { Up } \\
270\end{array}$ & $\begin{array}{l}.04 \\
.02 \\
.06\end{array}$ & $\begin{array}{l}--- \\
--- \\
--\end{array}$ \\
\hline & $\begin{array}{l}\text { Long Beach } \\
\text { VA Hospital } \\
\text { (VA) }\end{array}$ & $\begin{array}{r}33.78 \mathrm{~N} \\
118.12 \mathrm{~W}\end{array}$ & (3) & 5.1 & & & \\
\hline & Basement & & & & $\begin{array}{l}360 \\
\text { Up } \\
270\end{array}$ & $\begin{array}{l}.06 \\
.03 \\
.05\end{array}$ & $\begin{array}{l}--- \\
---\end{array}$ \\
\hline & 6th Floor & & & & $\begin{array}{l}360 \\
\text { Up } \\
270\end{array}$ & $\begin{array}{l}.09 \\
.05 \\
.10\end{array}$ & 1 peak \\
\hline & 11th Floor & & & & $\begin{array}{l}360 \\
\text { Up } \\
270\end{array}$ & $\begin{array}{l}.16 \\
.07 \\
.17\end{array}$ & $\begin{array}{l}1.7 \\
--- \\
1.2\end{array}$ \\
\hline
\end{tabular}


Table 1. Catalogue of National Cooperative Strong-Motion Network accelerograph records recovered c'uring 1987-Continued

\begin{tabular}{|c|c|c|c|c|c|c|c|}
\hline Earthquake & $\begin{array}{l}\text { Station name } \\
\text { (owner) }\end{array}$ & $\begin{array}{l}\text { Station } \\
\text { location } \\
\left({ }^{\circ}\right)\end{array}$ & $\begin{array}{l}\text { Trigger } \\
\text { time }\end{array}$ & $\begin{array}{l}\text { S-minus } \\
\text { trigger } \\
(\mathrm{s})\end{array}$ & $\begin{array}{l}\text { Direction } \\
\quad(a z)\end{array}$ & $\begin{array}{l}\text { Maximum } \\
\text { amplitude } \\
\text { (g) }\end{array}$ & $\begin{array}{l}\text { Duration } \\
\text { (s) }\end{array}$ \\
\hline \multirow[t]{15}{*}{$\begin{array}{l}4 \text { october } 1987 \\
1059: 38.1 \text { G.m.t. } \\
\text { Southern Calif. } \\
34.070 \mathrm{~N}, 118.100 \mathrm{~W} \\
\text { Magnitude } 5.3 \mathrm{ML}-- \\
\text { Continued }\end{array}$} & $\begin{array}{l}\text { Los Angeles } \\
\text { Wadsworth VA Hospital } \\
\text { (VA/USGS) } \\
\text { North Ground Site }\end{array}$ & $\begin{array}{r}34.050 \mathrm{~N} \\
118.452 \mathrm{~W}\end{array}$ & $59: 44.7$ & 5.0 & & (1) & \\
\hline & $\begin{array}{l}\text { Los Angeles, Brentwood } \\
\text { VA Hospital } \\
\text { (VA) }\end{array}$ & $\begin{array}{r}34.058 \mathrm{~N} \\
118.457 \mathrm{~W}\end{array}$ & (3) & 4.0 & & (1) & \\
\hline & $\begin{array}{l}\text { Sepulveda Dam } \\
\text { (ACOE) }\end{array}$ & $\begin{array}{r}34.17 \mathrm{~N} \\
118.47 \mathrm{~W}\end{array}$ & (3) & 5.0 & & & \\
\hline & Crest & & & & $\begin{array}{l}054 \\
\text { Up } \\
324\end{array}$ & $\begin{array}{l}.05 \\
.04 \\
.04\end{array}$ & $\begin{array}{l}--- \\
---\end{array}$ \\
\hline & Downstream & & & & $\begin{array}{l}054 \\
\text { Up } \\
324\end{array}$ & $\begin{array}{l}.04 \\
.05 \\
.04\end{array}$ & $\begin{array}{l}--- \\
--- \\
---\end{array}$ \\
\hline & $\begin{array}{l}\text { Santa Ana, Orange Cty. } \\
\text { Eng ineering Bldg., } \\
\text { Basement (USGS) }\end{array}$ & $\begin{array}{r}33.75 \mathrm{~N} \\
117.87 \mathrm{~W}\end{array}$ & (3) & (2) & & (1) & \\
\hline & $\begin{array}{l}\text { Jensen Filter Plant } \\
\text { (MWD) }\end{array}$ & $\begin{array}{r}34.309 \mathrm{~N} \\
118.499 \mathrm{~W}\end{array}$ & (3) & 6.4 & & & \\
\hline & Administration Bldg. & & & & & (1) & \\
\hline & Generator Room & & & & & (1) & \\
\hline & Reservoir Roof & & & & $\begin{array}{l}022 \\
\text { Up } \\
292\end{array}$ & $\begin{array}{l}.05 \\
.02 \\
.07\end{array}$ & $\begin{array}{l}--- \\
--- \\
---\end{array}$ \\
\hline & $\begin{array}{l}\text { Prado Dam } \\
(A C O E)\end{array}$ & $\begin{array}{r}33.89 \mathrm{~N} \\
117.64 \mathrm{~W}\end{array}$ & (3) & 5.6 & & & \\
\hline & Downstream & & & & $\begin{array}{l}090 \\
U p \\
360\end{array}$ & $\begin{array}{l}.04 \\
.03 \\
.07\end{array}$ & $\begin{array}{l}--- \\
--- \\
---\end{array}$ \\
\hline & $\begin{array}{l}\text { Valyermo Forest Sta. } \\
\text { Ground Level } \\
\text { (USGS) }\end{array}$ & $\begin{array}{r}34.44 \mathrm{~N} \\
117.85 \mathrm{~W}\end{array}$ & $59: 46.2$ & 6.2 & & (1) & \\
\hline & $\begin{array}{l}\text { Littlerock Post office } \\
\text { Ground Level } \\
\text { (USGS) }\end{array}$ & $\begin{array}{r}34.521 \mathrm{~N} \\
117.991 \mathrm{~W}\end{array}$ & (3) & (2) & & (1) & \\
\hline & $\begin{array}{l}\text { Palmdale Fire Station } \\
\text { Ground Level } \\
\text { (USGS) }\end{array}$ & $\begin{array}{r}34.58 \mathrm{~N} \\
118.11 \mathrm{~W}\end{array}$ & $59: 47.8$ & 6.5 & & (1) & \\
\hline
\end{tabular}


Table 1. Catalogue of National Cooperative Strong-Motion Network accelerograph records recove"ed during 1987-Continued

\begin{tabular}{|c|c|c|c|c|c|c|c|}
\hline Earthquake & $\begin{array}{l}\text { Station name } \\
\text { (owner) }\end{array}$ & $\begin{array}{l}\text { Station } \\
\text { location } \\
\left({ }^{\circ}\right)\end{array}$ & $\begin{array}{l}\text { Trigger } \\
\text { time }\end{array}$ & $\begin{array}{c}\text { S-minus } \\
\text { tr igger } \\
(\mathrm{s})\end{array}$ & $\begin{array}{l}\text { Direction } \\
\quad(\mathrm{az})\end{array}$ & $\begin{array}{l}\text { Maximurn } \\
\text { ampl itude } \\
\text { (g) }\end{array}$ & $\begin{array}{l}\text { Duration } \\
\text { (s) }\end{array}$ \\
\hline \multirow{3}{*}{$\begin{array}{l}1 \text { October } 1987- \\
4 \text { October } 1987 \\
\text { Southern Cal if. } \\
\text { Epicenters and } \\
\text { magnitudes unknown }\end{array}$} & $\begin{array}{l}\text { Los Angeles } \\
4407 \text { Jasper Street } \\
\text { (USGS) }\end{array}$ & $\begin{array}{r}34.081 \mathrm{~N} \\
118.188 \mathrm{~W}\end{array}$ & (3) & 1.9 & $\begin{array}{l}130 \\
\text { Up } \\
040\end{array}$ & $\begin{array}{l}.09 \\
.04 \\
.07\end{array}$ & $\begin{array}{l}--- \\
--- \\
---\end{array}$ \\
\hline & & & (3) & (2) & $\begin{array}{l}130 \\
\text { Up } \\
040\end{array}$ & $\begin{array}{l}.07 \\
.06 \\
.09\end{array}$ & $\begin{array}{l}--. \\
--- \\
--\end{array}$ \\
\hline & & & (3) & 2.1 & $\begin{array}{l}130 \\
\text { Up } \\
040\end{array}$ & $\begin{array}{l}.07 \\
.03 \\
.04\end{array}$ & $\begin{array}{l}--- \\
---\end{array}$ \\
\hline
\end{tabular}

\begin{tabular}{|c|c|c|c|c|c|c|c|}
\hline $\begin{array}{l}7 \text { 0ctober } 1987 \\
2032 \text { G.m.t. } \\
\text { Alaska } \\
\text { Epicenter and } \\
\text { magnitude unknown }\end{array}$ & $\begin{array}{l}\text { Guyot } \mathrm{Hills} \\
\text { (USGS) }\end{array}$ & $\begin{array}{r}60.146 \mathrm{~N} \\
141.472 \mathrm{~W}\end{array}$ & $32: 15.2^{4}$ & $(2)$ & & $(1)$ & \\
\hline \multirow{6}{*}{$\begin{array}{l}22 \text { 0ctober } 1987 \\
0348: 00.3 \mathrm{GMT} \\
\text { Central Cal if. } \\
37.805 \mathrm{~N}, 121.743 \mathrm{~W} \\
\text { Magn itude } 4.4 \mathrm{ML}\end{array}$} & $\begin{array}{l}\text { Livermore } \\
\text { VA Hospital } \\
\text { (VA) }\end{array}$ & $\begin{array}{r}37.625 \mathrm{~N} \\
121.762 \mathrm{~W}\end{array}$ & (3) & 2.0 & & & \\
\hline & Roof (7th floor) & & & & $\begin{array}{l}125 \\
\text { Up } \\
035\end{array}$ & $\begin{array}{l}.11 \\
.02 \\
.17\end{array}$ & $\begin{array}{l}1.1 \\
--- \\
1.3\end{array}$ \\
\hline & $\begin{array}{l}\text { Del Valle Dam } \\
\text { (CDWR) }\end{array}$ & $\begin{array}{c}37.615 \\
121.745 \mathrm{~W}\end{array}$ & (3) & $(2)$ & & & \\
\hline & Toe & & & & & $(1)$ & \\
\hline & Crest & & & & & $(1)$ & \\
\hline & Note: Event/record & correlation & questionab & Te. & & & \\
\hline \multirow{4}{*}{$\begin{array}{l}7 \text { November } 1987 \\
\text { 1506:01.2 G.m.t. } \\
\text { Central Cal if. } \\
36.583 \mathrm{~N}, 121.218 \mathrm{~W} \\
\text { Magnitude } 4.0 \mathrm{ML}\end{array}$} & $\begin{array}{l}\text { Bear Valley Station } 7 \\
\text { Pinnacles National } \\
\text { Monument (USGS) }\end{array}$ & $\begin{array}{r}36.483 \mathrm{~N} \\
121.180 \mathrm{~W}\end{array}$ & $06: 03.5$ & 1.6 & & (1) & \\
\hline & $\begin{array}{l}\text { Bear Valley Station } 10 \\
\text { Webb Residence } \\
\text { (USGS) }\end{array}$ & $\begin{array}{r}36.532 \mathrm{~N} \\
121.143 \mathrm{~W}\end{array}$ & $06: 03.7$ & 1.7 & $\begin{array}{l}310 \\
\text { Up } \\
220\end{array}$ & $\begin{array}{l}.06 \\
.03 \\
.09\end{array}$ & $\begin{array}{l}--- \\
--- \\
---\end{array}$ \\
\hline & $\begin{array}{l}\text { Bear Valley Station } 1 \\
\text { CDF Fire Station } \\
\text { (USGS) }\end{array}$ & $\begin{array}{r}36.573 \mathrm{~N} \\
121.184 \mathrm{~W}\end{array}$ & $06: 04.2$ & $(2)$ & & $(1)$ & \\
\hline & $\begin{array}{l}\text { Bear Valley Station } 6 \\
\text { James Ranch } \\
\text { (USGS) }\end{array}$ & $\begin{array}{r}36.504 \mathrm{~N} \\
121.101 \mathrm{~W}\end{array}$ & $06: 04.2$ & 2.4 & & $(1)$ & \\
\hline
\end{tabular}


Table 1. Catalogue of National Cooperative. Strong-Motion Network accelerograph records recovered during 1987-Continued

\begin{tabular}{|c|c|c|c|c|c|c|c|}
\hline Earthquake & $\begin{array}{l}\text { Station name } \\
\text { (owner) }\end{array}$ & $\begin{array}{l}\text { Station } \\
\text { location } \\
\left({ }^{\circ}\right)\end{array}$ & $\begin{array}{l}\text { Trigger } \\
\text { time }\end{array}$ & $\begin{array}{l}\text { S-minus } \\
\text { trigger } \\
(\mathrm{s})\end{array}$ & $\begin{array}{l}\text { Direction } \\
\quad(a z)\end{array}$ & $\begin{array}{l}\text { Maximum } \\
\text { amplitude } \\
(\mathrm{g})\end{array}$ & $\begin{array}{l}\text { Duratior } \\
\text { (s) }\end{array}$ \\
\hline $\begin{array}{l}7 \text { November } 1987 \\
1506: 01.2 \text { G.m.t. } \\
\text { Central Calif. } \\
36.583 \mathrm{~N}, 121.218 \mathrm{~W} \\
\text { Magnitude } 4.0 \mathrm{ML-} \\
\text { Cont inued }\end{array}$ & $\begin{array}{l}\text { Bear Valley Station } 5 \\
\text { Callens Ranch } \\
\text { (USGS) }\end{array}$ & $\begin{array}{r}36.673 \mathrm{~N} \\
121.195 \mathrm{~W}\end{array}$ & (3) & 2.3 & & (1) & \\
\hline \multirow{4}{*}{$\begin{array}{l}17 \text { November } 1987 \\
0846: 53.3 \text { G.m.t. } \\
\text { Southern A } 1 \text { aska } \\
58.586 \mathrm{~N}, 143.270 \mathrm{~W} \\
\text { Magnitude } 7.0 \mathrm{ML}\end{array}$} & $\begin{array}{l}\text { Sunshine Point } \\
\text { (USGS) }\end{array}$ & $\begin{array}{r}60.180 \mathrm{~N} \\
142.838 \mathrm{~W}\end{array}$ & $48: 16.2^{4}$ & (2) & & (1) & \\
\hline & $\begin{array}{l}\text { Kayak Is land } \\
\text { Ridge } \\
\text { (USGS) }\end{array}$ & $\begin{array}{r}59.927 \mathrm{~N} \\
144.500 \mathrm{~W}\end{array}$ & $47: 37.9^{4}$ & 0.4 & & $(1)$ & \\
\hline & $\begin{array}{l}\text { Guyot Hills } \\
\text { (USGS) }\end{array}$ & $\begin{array}{r}60.146 \mathrm{~N} \\
141.471 \mathrm{~W}\end{array}$ & $48: 20.9^{4}$ & (2) & & (1) & \\
\hline & $\begin{array}{l}\text { Yakutat } \\
\text { FAA VOR Building } \\
\text { (USGS) }\end{array}$ & $\begin{array}{r}59.51 \mathrm{~N} \\
139.65 \mathrm{~W}\end{array}$ & (3) & (2) & & (1) & \\
\hline \multirow{3}{*}{$\begin{array}{l}22 \text { November } 1987 \\
0411: 52.5 \text { G.m.t. } \\
\text { Northern Cal if. } \\
40.400 \mathrm{~N}, 124.423 \mathrm{~W} \\
\text { Magnitude } 3.7 \mathrm{ML}\end{array}$} & $\begin{array}{l}\text { Eel River Valley Array } \\
\text { Centerville Naval Fac. } \\
\text { (USGS) }\end{array}$ & $\begin{array}{r}40.563 \mathrm{~N} \\
124.348 \mathrm{~W}\end{array}$ & $11: 58.2$ & 4.9 & $\begin{array}{l}360 \\
\text { Up } \\
270\end{array}$ & $\begin{array}{l}.05 \\
.01 \\
.03\end{array}$ & $\begin{array}{l}--- \\
---\end{array}$ \\
\hline & $\begin{array}{l}\text { Eel River Valley Array } \\
\text { Ferndale Fire Station } \\
\text { (USGS) }\end{array}$ & $\begin{array}{r}40.58 \mathrm{~N} \\
124.26 \mathrm{~W}\end{array}$ & $11: 58.1$ & 5.5 & $\begin{array}{l}360 \\
\text { Up } \\
270\end{array}$ & $\begin{array}{l}.02 \\
.01 \\
.07\end{array}$ & $\begin{array}{l}--- \\
--- \\
--\end{array}$ \\
\hline & $\begin{array}{l}\text { Eel River Valley Array } \\
\text { Fortuna Fire Station } \\
\text { (USGS) }\end{array}$ & $\begin{array}{r}40.599 \mathrm{~W} \\
124.154 \mathrm{~W}\end{array}$ & (3) & (2) & & (1) & \\
\hline $\begin{array}{l}24 \text { November } 1987 \\
\text { 0132:48.0 G.m.t. } \\
\text { Southern Calif. } \\
33.070 \mathrm{~N}, 115.780 \mathrm{~W} \\
\text { Magnitude } 4.2 \mathrm{ML}\end{array}$ & $\begin{array}{l}\text { Salton Sea } \\
\text { Wildlife Refuge } \\
\text { (USGS) }\end{array}$ & $\begin{array}{r}33.18 \mathrm{~N} \\
115.62 \mathrm{~W}\end{array}$ & $32: 52.2$ & 3.0 & & (1) & \\
\hline \multirow{3}{*}{$\begin{array}{l}24 \text { November } 1987 \\
0154: 14.5 \text { G.m.t. } \\
\text { Southern Cal if. } \\
33.083 \mathrm{~N}, 115.775 \mathrm{~W} \\
\text { Magnitude } 6.2 \mathrm{Ms}\end{array}$} & $\begin{array}{l}\text { Superstition Mtn. } \\
\text { Camera Site } 8 \\
\text { (USGS) }\end{array}$ & $\begin{array}{r}32.955 \mathrm{~N} \\
115.823 \mathrm{~W}\end{array}$ & (3) & 1.6 & $\begin{array}{l}135 \\
\text { Up } \\
045\end{array}$ & $\begin{array}{l}.13 \\
.12 \\
.11\end{array}$ & $\begin{array}{r}4.8 \\
1-\text { peak } \\
2.6\end{array}$ \\
\hline & $\begin{array}{l}\text { Parachute Test Site } \\
\text { Imler Road } \\
\text { (USGS) }\end{array}$ & $\begin{array}{r}32.93 \mathrm{~N} \\
115.70 \mathrm{~W}\end{array}$ & $54: 19.2$ & 3.3 & $\begin{array}{l}315 \\
\text { Up } \\
225\end{array}$ & $\begin{array}{l}.10 \\
.10 \\
.13\end{array}$ & $\begin{array}{r}\text { 2-peaks } \\
1 \text {-peak } \\
3.3\end{array}$ \\
\hline & $\begin{array}{l}\text { Salton Sea } \\
\text { Wildlife Refuge } \\
\text { (USGS) }\end{array}$ & $\begin{array}{r}33.18 \mathrm{~N} \\
115.62 \mathrm{~W}\end{array}$ & $54: 18.8$ & 2.5 & $\begin{array}{l}315 \\
\text { Up } \\
225\end{array}$ & $\begin{array}{l}.18 \\
.20 \\
.13\end{array}$ & $\begin{array}{l}2.4 \\
3.9 \\
0.8\end{array}$ \\
\hline
\end{tabular}


Table 1. Catalogue of National Cooperative Strong-Motion Network accelerograph records recovered during 1987-Continued

\begin{tabular}{|c|c|c|c|c|c|c|c|}
\hline Earthquake & $\begin{array}{l}\text { Station name } \\
\text { (owner) }\end{array}$ & $\begin{array}{l}\text { Station } \\
\text { location } \\
\left({ }^{\circ}\right)\end{array}$ & $\begin{array}{l}\text { Trigger } \\
\text { time }\end{array}$ & $\begin{array}{l}\text { S-minus } \\
\text { trigger } \\
(\mathrm{s})\end{array}$ & $\begin{array}{l}\text { Direction } \\
\quad(a z)\end{array}$ & $\begin{array}{l}\text { Maximum } \\
\text { amplitud? } \\
(\text { g) }\end{array}$ & $\begin{array}{l}\text { Duration } \\
\text { (s) }\end{array}$ \\
\hline \multirow{13}{*}{$\begin{array}{l}24 \text { November } 1987 \\
0154: 14.5 \text { G.m.t. } \\
\text { Southern Cai if. } \\
33.083 \mathrm{~N}, 115.775 \mathrm{~W} \\
\text { Magnitude } 6.2 \mathrm{Ms-} \\
\text { Continued }\end{array}$} & $\begin{array}{l}\text { Imperial Wild life } \\
\text { Liquefact ion Array } \\
\text { (USGS) }\end{array}$ & $\begin{array}{r}33.10 \mathrm{~N} \\
115.53 \mathrm{~W}\end{array}$ & $54: 19.85$ & 5.8 & & & \\
\hline & $\begin{array}{l}\text { Channel } 1 \text { - surfac } \\
\text { Channel } 2 \text { - surfac } \\
\text { Channel } 3 \text { - surfac } \\
\text { Channel } 4-7.5 \mathrm{~m} \\
\text { Channel } 5-7.5 \mathrm{~m} \\
\text { Channel } 6-7.5 \mathrm{~m}\end{array}$ & $\begin{array}{l}\text { e } \\
\text { downhole } \\
\text { downhole } \\
\text { downhole }\end{array}$ & & & $\begin{array}{l}360 \\
\text { Up } \\
090 \\
360 \\
\text { Up } \\
090\end{array}$ & $\begin{array}{l}.13 \\
.18 \\
.13 \\
.08 \\
.09 \\
.08\end{array}$ & $\begin{array}{l}1.4 \\
2.7 \\
0.9 \\
--- \\
--. \\
---\end{array}$ \\
\hline & $\begin{array}{l}\text { Calipatria } \\
\text { Fire Station } \\
\text { (USGS) }\end{array}$ & $\begin{array}{r}33.13 \mathrm{~N} \\
115.52 \mathrm{~W}\end{array}$ & (3) & 6.0 & $\begin{array}{l}315 \\
\text { Up } \\
225\end{array}$ & $\begin{array}{l}.15 \\
.18 \\
.22\end{array}$ & $\begin{array}{l}0.6 \\
1.6 \\
0.7\end{array}$ \\
\hline & $\begin{array}{l}\text { Brawley } \\
\text { A irport Hangar } \\
\text { (USGS) }\end{array}$ & $\begin{array}{r}32.988 \mathrm{~N} \\
115.509 \mathrm{~W}\end{array}$ & $54: 23.8$ & 1.6 & $\begin{array}{l}315 \\
\text { Up } \\
225\end{array}$ & $\begin{array}{l}.06 \\
.04 \\
.06\end{array}$ & $\begin{array}{l}--- \\
---\end{array}$ \\
\hline & $\begin{array}{l}\text { Plaster City } \\
\text { Storehouse } \\
\text { (USGS) }\end{array}$ & $\begin{array}{r}32.79 \mathrm{~N} \\
115.86 \mathrm{~W}\end{array}$ & $54: 21.2$ & 4.2 & $\begin{array}{l}135 \\
\text { Up } \\
045\end{array}$ & $\begin{array}{l}.05 \\
.03 \\
.04\end{array}$ & $\begin{array}{l}--- \\
--- \\
---\end{array}$ \\
\hline & $\begin{array}{l}\text { Ocotillo Wells } \\
\text { Burro Bend Cafe } \\
\text { (USGS) }\end{array}$ & $\begin{array}{r}33.14 \mathrm{~N} \\
116.13 \mathrm{~W}\end{array}$ & $54: 22.5$ & 3.7 & $\begin{array}{l}315 \\
U p \\
225\end{array}$ & $\begin{array}{l}.03 \\
.04 \\
.03\end{array}$ & $\begin{array}{l}--- \\
---\end{array}$ \\
\hline & $\begin{array}{l}\text { E1 Centro Array } 10 \\
\text { Community Hospital } \\
\text { (USGS) }\end{array}$ & $\begin{array}{r}32.780 \mathrm{~N} \\
115.567 \mathrm{~W}\end{array}$ & (3) & 1.7 & $\begin{array}{l}230 \\
\text { Up } \\
140\end{array}$ & $\begin{array}{l}.04 \\
.03 \\
.05\end{array}$ & $\begin{array}{l}--- \\
---\end{array}$ \\
\hline & $\begin{array}{l}\text { E1 Centro Array } 9 \\
\text { Commercial Avenue } \\
\text { (USGS) }\end{array}$ & $\begin{array}{r}32.794 \mathrm{~N} \\
115.549 \mathrm{~W}\end{array}$ & (3) & 4.7 & $\begin{array}{l}360 \\
\text { Up } \\
270\end{array}$ & $\begin{array}{l}.04 \\
.03 \\
.05\end{array}$ & $\begin{array}{l}--- \\
--- \\
---\end{array}$ \\
\hline & $\begin{array}{l}\text { E1 Centro Array } 8 \\
\text { Cruickshank Road } \\
\text { (USGS) }\end{array}$ & $\begin{array}{r}32.811 \mathrm{~N} \\
115.532 \mathrm{~W}\end{array}$ & $54: 22.9$ & 5.9 & $\begin{array}{l}230 \\
\text { Up } \\
140\end{array}$ & $\begin{array}{l}.06 \\
.04 \\
.04\end{array}$ & $\begin{array}{l}--- \\
--- \\
--\end{array}$ \\
\hline & $\begin{array}{l}\text { El Centro Array } 7 \\
\text { Imp. Val ley College } \\
\text { (USGS) }\end{array}$ & $\begin{array}{r}32.829 \mathrm{~N} \\
115.504 \mathrm{~W}\end{array}$ & $54: 23.2$ & 5.6 & $\begin{array}{l}230 \\
\text { Up } \\
140\end{array}$ & $\begin{array}{l}.05 \\
.03 \\
.03\end{array}$ & $\begin{array}{l}-- \\
-- \\
--\end{array}$ \\
\hline & $\begin{array}{l}\text { E1 Centro Array } 6 \\
\text { Huston Road } \\
\text { (USGS) }\end{array}$ & $\begin{array}{r}32.839 \mathrm{~N} \\
115.487 \mathrm{~W}\end{array}$ & $54: 23.6$ & 5.5 & $\begin{array}{l}230 \\
\text { Up } \\
140\end{array}$ & $\begin{array}{l}.05 \\
.02 \\
.05\end{array}$ & $\begin{array}{l}--- \\
---\end{array}$ \\
\hline & $\begin{array}{l}\text { E1 Centro Array } 5 \\
\text { James Road } \\
\text { (USGS) }\end{array}$ & $\begin{array}{r}32.855 \mathrm{~N} \\
115.466 \mathrm{~W}\end{array}$ & $54: 24.0$ & 5.5 & $\begin{array}{l}230 \\
\text { Up } \\
140\end{array}$ & $\begin{array}{l}.11 \\
.02 \\
.08\end{array}$ & $\begin{array}{l}1-\text { peak } \\
--- \\
--\end{array}$ \\
\hline & $\begin{array}{l}\text { El Centro Diff. Array } \\
\text { Dogwood Road } \\
\text { (USGS) }\end{array}$ & $\begin{array}{r}32.796 \mathrm{~N} \\
115.535 \mathrm{~W}\end{array}$ & $54: 23.7$ & 5.5 & $\begin{array}{l}360 \\
\text { Up } \\
270\end{array}$ & $\begin{array}{l}.07 \\
.03 \\
.07\end{array}$ & $\begin{array}{l}--- \\
--- \\
--\end{array}$ \\
\hline
\end{tabular}


Table 1. Catalogue of National Cooperative Strong-Motion Network accelerograph records recovered during 1987-Continued

\begin{tabular}{|c|c|c|c|c|c|c|c|}
\hline Earthquake & $\begin{array}{l}\text { Station name } \\
\text { (owner) }\end{array}$ & $\begin{array}{l}\text { Station } \\
\text { location } \\
\left({ }^{\circ}\right)\end{array}$ & $\begin{array}{l}\text { Trigger } \\
\text { time }\end{array}$ & $\begin{array}{l}\text { S-minus } \\
\text { trigger } \\
(\mathrm{s})\end{array}$ & $\begin{array}{l}\text { Direction } \\
\quad(a z)\end{array}$ & $\begin{array}{l}\text { Maximum } \\
\text { ampli tude } \\
(\mathrm{g})\end{array}$ & $\begin{array}{l}\text { Duration } \\
\text { (s) }\end{array}$ \\
\hline \multirow{10}{*}{$\begin{array}{l}24 \text { November } 1987 \\
0154: 14.5 \text { G.m.t. } \\
\text { Southern Calif. } \\
33.083 \mathrm{~N}, 115.775 \mathrm{~W} \\
\text { Magnitude } 6.2 \mathrm{Ms}-- \\
\text { Continued }\end{array}$} & $\begin{array}{l}\text { El Centro Array } 4 \\
\text { Anderson Road } \\
\text { (USGS) }\end{array}$ & $\begin{array}{r}32.864 \mathrm{~N} \\
115.432 \mathrm{~W}\end{array}$ & $54: 30.9$ & (2) & $\begin{array}{l}230 \\
\text { Up } \\
140\end{array}$ & $\begin{array}{l}.03 \\
.02 \\
.02\end{array}$ & $\begin{array}{l}--\cdot \\
--- \\
--\end{array}$ \\
\hline & $\begin{array}{l}\text { E1 Centro Array } 11 \\
\text { MCCabe School } \\
\text { (USGS) }\end{array}$ & $\begin{array}{r}32.752 \mathrm{~N} \\
115.594 \mathrm{~W}\end{array}$ & $54: 23.7$ & 5.2 & $\begin{array}{l}230 \\
\text { Up } \\
140\end{array}$ & $\begin{array}{l}.05 \\
.03 \\
.04\end{array}$ & $\begin{array}{l}--- \\
--- \\
---\end{array}$ \\
\hline & $\begin{array}{l}\text { El Centro } \\
\text { Meadows Union School } \\
\text { (USGS) }\end{array}$ & $\begin{array}{r}32.800 \mathrm{~N} \\
115.473 \mathrm{~W}\end{array}$ & $54: 23.6$ & 6.7 & $\begin{array}{l}230 \\
\text { Up } \\
140\end{array}$ & $\begin{array}{l}.05 \\
.02 \\
.05\end{array}$ & $\begin{array}{l}--- \\
--- \\
---\end{array}$ \\
\hline & $\begin{array}{l}\text { El Centro Array } 12 \\
\text { Brockman Road } \\
\text { (USGS) }\end{array}$ & $\begin{array}{r}32.718 \mathrm{~N} \\
115.637 \mathrm{~W}\end{array}$ & $54: 28.5$ & 1.7 & $\begin{array}{l}230 \\
\text { Up } \\
140\end{array}$ & $\begin{array}{l}.05 \\
.01 \\
.04\end{array}$ & $\begin{array}{l}--- \\
---\end{array}$ \\
\hline & $\begin{array}{l}\text { Coachella Canal } \\
\text { Station } 3 \\
\text { (USGS) }\end{array}$ & $\begin{array}{r}33.51 \mathrm{~N} \\
115.77 \mathrm{~W}\end{array}$ & (3) & 6.8 & $\begin{array}{l}135 \\
\text { Up } \\
045\end{array}$ & $\begin{array}{l}.04 \\
.04 \\
.03\end{array}$ & $\begin{array}{l}--- \\
--- \\
--\end{array}$ \\
\hline & $\begin{array}{l}\text { Calexico } \\
\text { Fire Station } \\
\text { (USGS) }\end{array}$ & $\begin{array}{r}32.669 \mathrm{~N} \\
115.492 \mathrm{~W}\end{array}$ & (3) & 7.4 & $\begin{array}{l}315 \\
\text { Up } \\
225\end{array}$ & $\begin{array}{l}.04 \\
.03 \\
.05\end{array}$ & $\begin{array}{l}--- \\
---\end{array}$ \\
\hline & $\begin{array}{l}\text { Coachella Canal } \\
\text { Station } 2 \\
\text { (USGS) }\end{array}$ & $\begin{array}{r}33.56 \mathrm{~N} \\
115.95 \mathrm{~W}\end{array}$ & (3) & (2) & $\begin{array}{l}135 \\
\text { Up } \\
045\end{array}$ & $\begin{array}{l}.03 \\
.02 \\
.02\end{array}$ & $\begin{array}{l}--- \\
---\end{array}$ \\
\hline & $\begin{array}{l}\text { Bonds Corner (SMA) } \\
\text { Highway } 98 \text { at } 115 \\
\text { (USGS) }\end{array}$ & $\begin{array}{r}32.693 \mathrm{~N} \\
115.338 \mathrm{~W}\end{array}$ & $54: 29.7$ & 5.6 & $\begin{array}{l}230 \\
\text { Up } \\
140\end{array}$ & $\begin{array}{l}.09 \\
.02 \\
.06\end{array}$ & $\begin{array}{l}--- \\
---\end{array}$ \\
\hline & $\begin{array}{l}\text { Bonds Corner (RFT) } \\
\text { Highway } 98 \text { at } 115 \\
\text { (USGS) }\end{array}$ & $\begin{array}{r}32.693 \mathrm{~N} \\
115.338 \mathrm{~W}\end{array}$ & $54: 29.7$ & 5.6 & $\begin{array}{l}230 \\
\text { Up } \\
140\end{array}$ & $\begin{array}{l}.09 \\
.02 \\
.06\end{array}$ & $\begin{array}{l}--- \\
---\end{array}$ \\
\hline & $\begin{array}{l}\text { Coachella Canal } \\
\text { Station } 1 \\
\text { (USGS) }\end{array}$ & $\begin{array}{r}33.64 \mathrm{~N} \\
116.08 \mathrm{~W}\end{array}$ & (3) & 10.0 & $\begin{array}{l}135 \\
\text { Up } \\
045\end{array}$ & $\begin{array}{l}.08 \\
.05 \\
.08\end{array}$ & $\begin{array}{l}--- \\
--- \\
--\end{array}$ \\
\hline $\begin{array}{l}24 \text { Nov.ember } 1987 \\
\text { 0157 G.m.t. } \\
\text { Southern Cal if. } \\
\text { Epicenter and } \\
\text { magnitude unknown }\end{array}$ & $\begin{array}{l}\text { Salton Sea } \\
\text { Wildlife Refuge } \\
\text { (USGS) }\end{array}$ & $\begin{array}{r}33.18 \mathrm{~N} \\
115.62 \mathrm{~W}\end{array}$ & $57: 31.9$ & 0.7 & $\begin{array}{l}315 \\
\text { Up } \\
225\end{array}$ & $\begin{array}{l}.19 \\
.09 \\
.10\end{array}$ & $\begin{array}{l}0.2 \\
--- \\
1 \text { peak }\end{array}$ \\
\hline $\begin{array}{l}24 \text { November } 1987 \\
0201: 46.7 \text { G.m.t. } \\
\text { Southern Calif. } \\
33.070 \mathrm{~N}, 115.790 \mathrm{~W} \\
\text { Magnitude } 3.8 \mathrm{ML}\end{array}$ & $\begin{array}{l}\text { Salton Sea } \\
\text { Wildlife Refuge } \\
\text { (USGS) }\end{array}$ & $\begin{array}{r}33.18 \mathrm{~N} \\
115.62 \mathrm{~W}\end{array}$ & $01: 51.3$ & 3.4 & & (1) & \\
\hline
\end{tabular}


Table 1. Catalogue of National Cooperative Strong-Motion Network accelerograph records recovered during 1987-Continued

\begin{tabular}{|c|c|c|c|c|c|c|c|}
\hline Earthquake & $\begin{array}{l}\text { Station name } \\
\text { (owner) }\end{array}$ & $\begin{array}{l}\text { Station } \\
\text { location } \\
\left({ }^{\circ}\right)\end{array}$ & $\begin{array}{l}\text { Trigger } \\
\text { time }\end{array}$ & $\begin{array}{l}\text { S-minus } \\
\text { tr igger } \\
\quad(s)\end{array}$ & $\begin{array}{l}\text { Direction } \\
\quad(a z)\end{array}$ & $\begin{array}{l}\text { Maximun } \\
\text { amplitude } \\
\text { (g) }\end{array}$ & $\begin{array}{l}\text { Duration } \\
\text { (s) }\end{array}$ \\
\hline $\begin{array}{l}24 \text { November } 1987 \\
0215: 26 \text { G.m.t. } \\
\text { Southern Cal if. } \\
33.25 \mathrm{~N}, 115.62 \mathrm{~W} \\
\text { Magnitude } 4.3 \mathrm{MB}\end{array}$ & $\begin{array}{l}\text { Salton Sea } \\
\text { Wildlife Refuge } \\
\text { (USGS) }\end{array}$ & $\begin{array}{r}33.18 \mathrm{~N} \\
115.62 \mathrm{~W}\end{array}$ & $15: 31.6$ & (2) & & (1) & \\
\hline $\begin{array}{l}24 \text { November } 1987 \\
0218 \text { G.m.t. } \\
\text { Southern Calif. } \\
\text { Epicenter and } \\
\text { magnitude unknown }\end{array}$ & $\begin{array}{l}\text { Salton Sea } \\
\text { Wildlife Refuge } \\
\text { (USGS) }\end{array}$ & $\begin{array}{r}33.18 \mathrm{~N} \\
115.62 \mathrm{~W}\end{array}$ & $18: 23.7$ & 0.4 & & (1) & \\
\hline $\begin{array}{l}24 \text { November } 1987 \\
0225 \text { G.m.t. } \\
\text { Southern Calif. } \\
\text { Epicenter and } \\
\text { magnitude unknown }\end{array}$ & $\begin{array}{l}\text { Salton Sea } \\
\text { Wildlife Refuge } \\
\text { (USGS) }\end{array}$ & $\begin{array}{r}33.18 \mathrm{~N} \\
115.62 \mathrm{~W}\end{array}$ & $25: 15.0$ & 0.6 & & (1) & \\
\hline $\begin{array}{l}24 \text { November } 1987 \\
0225: 52.0 \mathrm{G} . \mathrm{m} . \mathrm{t} . \\
\text { Southern Calif. } \\
33.080 \mathrm{~N}, 115.780 \mathrm{~W} \\
\text { Magnitude } 3.5 \mathrm{ML}\end{array}$ & $\begin{array}{l}\text { Salton Sea } \\
\text { Wildlife Refuge } \\
\text { (USGS) }\end{array}$ & $\begin{array}{r}33.18 \mathrm{~N} \\
115.62 \mathrm{~W}\end{array}$ & {$[25: 53.2]$} & {$[1.8]$} & $\begin{array}{l}315 \\
\text { Up } \\
225\end{array}$ & $\begin{array}{l}.05 \\
.03 \\
.05\end{array}$ & $\begin{array}{l}--- \\
--- \\
--\end{array}$ \\
\hline $\begin{array}{l}24 \text { November } 1987 \\
0226 \text { G.m.t. } \\
\text { Southern Calif. } \\
\text { Epicenter and } \\
\text { magnitude unknown }\end{array}$ & $\begin{array}{l}\text { Salton Sea } \\
\text { Wildlife Refuge } \\
\text { (USGS) }\end{array}$ & $\begin{array}{r}33.18 \mathrm{~N} \\
115.62 \mathrm{~W}\end{array}$ & {$[26: 34.5]$} & ] $[1.4]$ & & (1) & \\
\hline $\begin{array}{l}24 \text { November } 1987 \\
0249 \text { G.m.t. } \\
\text { Southern Calif. } \\
\text { Epicenter and } \\
\text { magnitude unknown }\end{array}$ & $\begin{array}{l}\text { Salton Sea } \\
\text { Wildlife Refuge } \\
\text { (USGS) }\end{array}$ & $\begin{array}{r}33.18 \mathrm{~N} \\
115.62 \mathrm{~W}\end{array}$ & $49: 30.2$ & 0.5 & & (1) & \\
\hline $\begin{array}{l}24 \text { November } 1987 \\
0253: 00.7 \text { G.m.t. } \\
\text { Southern Cal if. } \\
33.040 \mathrm{~N}, 115.810 \mathrm{~W} \\
\text { Magnitude } 4.7 \mathrm{ML}\end{array}$ & $\begin{array}{l}\text { E1 Centro } \\
\text { Parachute Test Site } \\
\text { (USGS) }\end{array}$ & $\begin{array}{r}32.93 \mathrm{~N} \\
115.70 \mathrm{~W}\end{array}$ & $53: 04.8$ & 3.2 & & (1) & \\
\hline $\begin{array}{l}24 \text { November } 1987 \\
0321: 10.3 \mathrm{G} . \mathrm{m} . \mathrm{t} . \\
\text { Southern Cal if. } \\
33.170 \mathrm{~N}, 115.660 \mathrm{~W} \\
\text { Magnitude } 3.0 \mathrm{ML}\end{array}$ & $\begin{array}{l}\text { Salton Sea } \\
\text { Wildlife Refuge } \\
\text { (USGS) }\end{array}$ & $\begin{array}{r}33.18 \mathrm{~N} \\
115.62 \mathrm{~W}\end{array}$ & $21: 12.8$ & 0.5 & $\begin{array}{l}315 \\
\text { Up } \\
225\end{array}$ & $\begin{array}{l}.05 \\
.03 \\
.04\end{array}$ & $\begin{array}{l}--- \\
--- \\
---\end{array}$ \\
\hline $\begin{array}{l}24 \text { November } 1987 \\
0323: 24.7 \text { G.m.t. } \\
\text { Southern Cal if. } \\
33.180 \mathrm{~N}, 115.650 \mathrm{~W} \\
\text { Magn itude } 3.2 \mathrm{ML}\end{array}$ & $\begin{array}{l}\text { Salton Sea } \\
\text { Wildlife Refuge } \\
\text { (USGS) }\end{array}$ & $\begin{array}{r}33.18 \mathrm{~N} \\
115.62 \mathrm{~W}\end{array}$ & $23: 26.5$ & 0.2 & & (1) & \\
\hline
\end{tabular}


Table 1. Catalogue of National Cooperative Strong-Motion Network accelerograph records recovered during 1987-Continued

\begin{tabular}{|c|c|c|c|c|c|c|c|}
\hline Earthquake & $\begin{array}{l}\text { Station name } \\
\text { (owner) }\end{array}$ & $\begin{array}{l}\text { Station } \\
\text { location } \\
\left({ }^{\circ}\right)\end{array}$ & $\begin{array}{l}\text { Trigger } \\
\text { time }\end{array}$ & $\begin{array}{l}\text { S-minus } \\
\text { trigger } \\
\text { (s) }\end{array}$ & $\begin{array}{l}\text { Direction } \\
\quad(a z)\end{array}$ & $\begin{array}{l}\text { Max imum } \\
\text { ampli itude } \\
(\mathrm{g})\end{array}$ & $\begin{array}{l}\text { Duration } \\
\text { (s) }\end{array}$ \\
\hline $\begin{array}{l}24 \text { November } 1987 \\
0324: 13.8 \text { G.m.t. } \\
\text { Southern Calif. } \\
33.180 \mathrm{~N}, 115.660 \mathrm{~W} \\
\text { Magnitude } 3.4 \mathrm{ML}\end{array}$ & $\begin{array}{l}\text { Salton Sea } \\
\text { Wildlife Refuge }\end{array}$ & $\begin{array}{r}33.18 \mathrm{~N} \\
115.62 \mathrm{~W}\end{array}$ & {$[24: 14.7]$} & {$[1.7]$} & & (1) & \\
\hline $\begin{array}{l}24 \text { November } 1987 \\
0404: 36.8 \text { G.m.t. } \\
\text { Southern Cal if. } \\
33.180 \mathrm{~N}, 115.650 \mathrm{~W} \\
\text { Magnitude } 3.6 \mathrm{ML}\end{array}$ & $\begin{array}{l}\text { Salton Sea } \\
\text { Wildlife Refuge } \\
\text { (USGS) }\end{array}$ & $\begin{array}{r}33.18 \mathrm{~N} \\
115.62 \mathrm{~W}\end{array}$ & $04: 37.7$ & 1.1 & $\begin{array}{l}315 \\
\text { Up } \\
225\end{array}$ & $\begin{array}{l}.13 \\
.09 \\
.12\end{array}$ & $\begin{array}{l}2 \text { fraks } \\
-0.1\end{array}$ \\
\hline $\begin{array}{l}24 \text { November } 1987 \\
0623: 23.1 \text { G.m.t. } \\
\text { Southern Cailif. } \\
33.020 \mathrm{~N}, 115.810 \mathrm{~W} \\
\text { Magnitude } 4.0 \mathrm{ML}\end{array}$ & $\begin{array}{l}\text { Imperial Wildlife } \\
\text { Liquefaction Array } \\
\text { (USGS) }\end{array}$ & $\begin{array}{r}33.01 \mathrm{~N} \\
115.53 \mathrm{~W}\end{array}$ & $23: 28.8$ & 4.9 & & (1) & \\
\hline \multirow{2}{*}{$\begin{array}{l}24 \text { November } 1987 \\
0155-1315 \text { G.m.t. } \\
\text { Southern Calif. } \\
\text { Epicenters and } \\
\text { magnitudes unknown }\end{array}$} & $\begin{array}{l}\text { Superstition Mountain } \\
\text { Camera Site } 8 \\
\text { (USGS) }\end{array}$ & $\begin{array}{r}32.955 \mathrm{~N} \\
115.823 \mathrm{~W}\end{array}$ & (3) & (2) & $\begin{array}{l}135 \\
\text { Up } \\
045\end{array}$ & $\begin{array}{l}.05 \\
.03 \\
.03\end{array}$ & --- \\
\hline & & & (3) & 0.1 & $\begin{array}{l}135 \\
\text { Up } \\
045\end{array}$ & $\begin{array}{l}.12 \\
.07 \\
.07\end{array}$ & $\begin{array}{l}1 \text { foak } \\
\cdots-\end{array}$ \\
\hline
\end{tabular}

Note: Four additional records ${ }^{1}$ recovered at Superstition Mountain Camerc Site 8.

\begin{tabular}{|c|c|c|c|c|c|c|c|}
\hline $\begin{array}{l}24 \text { November } 1987 \\
0400-1315 \text { G.m.t. } \\
\text { Southern Calif. } \\
\text { Epicenter and } \\
\text { magnitude unknown }\end{array}$ & $\begin{array}{l}\text { Calipatria } \\
\text { Fire Station } \\
\text { (USGS) }\end{array}$ & $\begin{array}{r}33.13 \mathrm{~N} \\
115.52 \mathrm{~W}\end{array}$ & (3) & 5.0 & & $(1)$ & \\
\hline \multirow{5}{*}{$\begin{array}{l}24 \text { November } 1987 \\
\text { 1315:56.4 G.m.t. } \\
\text { Southern Calif. } \\
33.010 \mathrm{~N}, 115.840 \mathrm{~W} \\
\text { Magn itude } 6.6 \mathrm{Ms}\end{array}$} & $\begin{array}{l}\text { Superstition Mountain } \\
\text { Camera Site } 8 \\
\text { (USGS) }\end{array}$ & $\begin{array}{r}32.955 \mathrm{~N} \\
115.823 \mathrm{~W}\end{array}$ & $15: 57.8$ & 0.1 & $\begin{array}{l}135 \\
\text { Up } \\
045\end{array}$ & $\begin{array}{l}.91 \\
.65 \\
.73\end{array}$ & $\begin{array}{l}16.5 \\
16.9 \\
15.8\end{array}$ \\
\hline & $\begin{array}{l}\text { Salton Sea, POE } \\
\text { Poe Rd. (Temp.) } \\
\text { (CIT) }\end{array}$ & $\begin{array}{r}33.097 \mathrm{~N} \\
115.751 \mathrm{~W}\end{array}$ & $16: 00.7$ & 2.6 & $\begin{array}{l}180 \\
\text { Up } \\
090\end{array}$ & $\begin{array}{l}.33 \\
.51 \\
.54\end{array}$ & $\begin{array}{l}14.8 \\
17.3 \\
18.1\end{array}$ \\
\hline & $\begin{array}{l}\text { Parachute Test Site } \\
\text { Imler Road } \\
\text { (USGS) }\end{array}$ & $\begin{array}{r}32.93 \mathrm{~N} \\
115.70 \mathrm{~W}\end{array}$ & $16: 01.2$ & 2.5 & $\begin{array}{l}315 \\
\text { Up } \\
225\end{array}$ & $\begin{array}{l}.53 \\
.45 \\
.49\end{array}$ & $\begin{array}{l}11.3 \\
14.5 \\
13.7\end{array}$ \\
\hline & $\begin{array}{l}\text { Salton Sea, KNB } \\
\text { Kornbloom Rd. (Temp.) } \\
\text { (CIT) }\end{array}$ & $\begin{array}{r}33.125 \mathrm{~N} \\
115.665 \mathrm{~W}\end{array}$ & $16: 02.6$ & 0.8 & $\begin{array}{l}180 \\
\text { Up } \\
090\end{array}$ & $\begin{array}{l}.19 \\
.18 \\
.15\end{array}$ & $\begin{array}{l}7.6 \\
1.5 \\
8.8\end{array}$ \\
\hline & $\begin{array}{l}\text { Plaster City } \\
\text { Storehouse } \\
\text { (USGS) }\end{array}$ & $\begin{array}{r}32.79 \mathrm{~N} \\
115.86 \mathrm{~W}\end{array}$ & $16: 01.2$ & 3.6 & $\begin{array}{l}135 \\
\text { Up } \\
045\end{array}$ & $\begin{array}{l}.19 \\
.11 \\
.15\end{array}$ & $\begin{array}{l}10.4 \\
1-\text { peak } \\
2.5\end{array}$ \\
\hline
\end{tabular}



1987-Continued

\begin{tabular}{|c|c|c|c|c|c|c|c|}
\hline Earthquake & $\begin{array}{l}\text { Station name } \\
\text { (owner) }\end{array}$ & $\begin{array}{l}\text { Station } \\
\text { location } \\
\left({ }^{\circ}\right)\end{array}$ & $\begin{array}{l}\text { Trigger } \\
\text { time }\end{array}$ & $\begin{array}{l}\text { S-minus } \\
\text { trigger } \\
(\mathrm{s})\end{array}$ & $\begin{array}{l}\text { Direction } \\
(\mathrm{az})\end{array}$ & $\begin{array}{l}\text { Maximum } \\
\text { amplitude } \\
\text { (g) }\end{array}$ & $\begin{array}{l}\text { Duration } \\
\text { (s) }\end{array}$ \\
\hline \multirow{12}{*}{$\begin{array}{l}24 \text { November } 1987 \\
1315: 56.4 \text { G.m.t. } \\
\text { Southern Calif. } \\
33.010 \mathrm{~N}, 115.840 \mathrm{~W} \\
\text { Magnitude } 6.6 \mathrm{Ms--} \\
\text { Cont inued }\end{array}$} & $\begin{array}{l}\text { Ocotillo Wells } \\
\text { Burro Bend Cafe } \\
\text { (USGS) }\end{array}$ & $\begin{array}{r}33.14 \mathrm{~N} \\
116.13 \mathrm{~W}\end{array}$ & $16: 04.0$ & 2.9 & $\begin{array}{l}315 \\
\text { Up } \\
225\end{array}$ & $\begin{array}{l}.11 \\
.09 \\
.08\end{array}$ & $\begin{array}{l}1-\text { peak } \\
-\cdots\end{array}$ \\
\hline & $\begin{array}{l}\text { Salton Sea } \\
\text { Wildlife Refuge } \\
\text { USGS) }\end{array}$ & $\begin{array}{r}33.18 \mathrm{~N} \\
115.62 \mathrm{~W}\end{array}$ & $16: 02.6$ & 3.5 & $\begin{array}{l}315 \\
\text { Up } \\
225\end{array}$ & $\begin{array}{l}.15 \\
.17 \\
.14\end{array}$ & $\begin{array}{r}9.2 \\
12.1 \\
11.6\end{array}$ \\
\hline & $\begin{array}{l}\text { Imperial Wildlife } \\
\text { Liquefaction Array } \\
\text { (USGS) }\end{array}$ & $\begin{array}{r}33.097 \mathrm{~N} \\
115.530 \mathrm{~W}\end{array}$ & $16: 03.45$ & 5.1 & & & \\
\hline & \multicolumn{2}{|c|}{$\begin{array}{l}\text { Channel } 1 \text { - surface } \\
\text { Channel } 2 \text { - surface } \\
\text { Channel } 3 \text { - surface } \\
\text { Channel } 4 \text { - } 7.5 \mathrm{~m} \text { downhole } \\
\text { Channel } 5-7.5 \mathrm{~m} \text { downhole } \\
\text { Channel } 6-7.5 \mathrm{~m} \text { downhole }\end{array}$} & & & $\begin{array}{l}360 \\
\text { Up } \\
090 \\
360 \\
\text { Up } \\
090\end{array}$ & $\begin{array}{l}.21 \\
.44 \\
.19 \\
.16 \\
.11 \\
.08\end{array}$ & $\begin{array}{l}13.2 \\
10.4 \\
5.9 \\
1.9 \\
1 \text { peak } \\
1 \text { peak }\end{array}$ \\
\hline & $\begin{array}{l}\text { Brawley } \\
\text { A irport Hangar } \\
\text { (USGS) }\end{array}$ & $\begin{array}{r}32.988 \mathrm{~N} \\
115.509 \mathrm{~W}\end{array}$ & $16: 14.1$ & 3.8 & $\begin{array}{l}315 \\
\text { Up } \\
225\end{array}$ & $\begin{array}{l}.15 \\
.13 \\
.15\end{array}$ & $\begin{array}{l}9.5 \\
3.6 \\
2.4\end{array}$ \\
\hline & $\begin{array}{l}\text { Calipatria } \\
\text { Fire Station } \\
\text { (USGS) }\end{array}$ & $\begin{array}{r}33.13 \mathrm{~N} \\
115.52 \mathrm{~W}\end{array}$ & (3) & 5.0 & $\begin{array}{l}315 \\
\text { Up } \\
225\end{array}$ & $\begin{array}{l}.32 \\
.21 \\
.24\end{array}$ & $\begin{array}{l}10.7 \\
10.8 \\
10.8\end{array}$ \\
\hline & $\begin{array}{l}\text { El Centro Array } 13 \\
\text { Strobel Residence } \\
\text { (USGS) }\end{array}$ & $\begin{array}{r}32.709 \mathrm{~N} \\
115.683 \mathrm{~W}\end{array}$ & $16: 04.7$ & 5.0 & $\begin{array}{l}230 \\
\text { Up } \\
140\end{array}$ & $\begin{array}{l}.19 \\
.07 \\
.17\end{array}$ & $\begin{array}{l}0.8 \\
--- \\
0.7\end{array}$ \\
\hline & $\begin{array}{l}\text { El Centro Array } 10 \\
\text { Community Hospital } \\
\text { (USGS) }\end{array}$ & $\begin{array}{r}32.780 \mathrm{~N} \\
115.567 \mathrm{~W}\end{array}$ & (3) & 4.3 & $\begin{array}{l}230 \\
\text { Up } \\
140\end{array}$ & $\begin{array}{l}.27 \\
.07 \\
.22\end{array}$ & $\begin{array}{l}7.6 \\
--- \\
9.3\end{array}$ \\
\hline & $\begin{array}{l}\text { El Centro Array } 11 \\
\text { MCCabe School } \\
\text { (USGS) }\end{array}$ & $\begin{array}{r}32.752 \mathrm{~N} \\
115.594 \mathrm{~W}\end{array}$ & $16: 04.8$ & 5.8 & $\begin{array}{l}230 \\
\text { Up } \\
140\end{array}$ & $\begin{array}{l}.32 \\
.13 \\
.21\end{array}$ & $\begin{array}{r}15.7 \\
6.9 \\
9.4\end{array}$ \\
\hline & $\begin{array}{l}\text { E1 Centro Array } 9 \\
\text { Commercial Avenue } \\
\text { (USGS) }\end{array}$ & $\begin{array}{r}32.794 \mathrm{~N} \\
115.549 \mathrm{~W}\end{array}$ & (3) & 4.6 & $\begin{array}{l}360 \\
\text { Up } \\
270\end{array}$ & $\begin{array}{l}.30 \\
.15 \\
.20\end{array}$ & $\begin{array}{l}3.6 \\
2.6 \\
2.8\end{array}$ \\
\hline & $\begin{array}{l}\text { El Centro Array } 8 \\
\text { E. Cruickshank Road } \\
\text { (USGS) }\end{array}$ & $\begin{array}{r}32.811 \mathrm{~N} \\
115.532 \mathrm{~W}\end{array}$ & $16: 04.9$ & 5.3 & $\begin{array}{l}230 \\
\text { Up } \\
140\end{array}$ & $\begin{array}{l}.35 \\
.21 \\
.33\end{array}$ & $\begin{array}{r}10.1 \\
4.9 \\
10.1\end{array}$ \\
\hline & $\begin{array}{l}\text { El Centro Array } 12 \\
\text { Brockman Road } \\
\text { (USGS) }\end{array}$ & $\begin{array}{r}32.718 \mathrm{~N} \\
115.637 \mathrm{~W}\end{array}$ & (3) & 5.4 & $\begin{array}{l}230 \\
\text { Up } \\
140\end{array}$ & $\begin{array}{l}.27 \\
.08 \\
.19\end{array}$ & $\begin{array}{l}2.0 \\
--2 \\
2.0\end{array}$ \\
\hline
\end{tabular}

Note: Instrument malfunction, partial record (approx. 10 seconds). 
Table 1. Catalogue of National Cooperative Strong-Motion Network accelerograph records recovered during 1987-Continued

\begin{tabular}{|c|c|c|c|c|c|c|c|}
\hline Earthquake & $\begin{array}{l}\text { Station name } \\
\text { (owner) }\end{array}$ & $\begin{array}{l}\text { Station } \\
\text { location } \\
\left({ }^{\circ}\right)\end{array}$ & $\begin{array}{l}\text { Trigger } \\
\text { time }\end{array}$ & $\begin{array}{l}\text { S-minus } \\
\text { trigger } \\
(\mathrm{s})\end{array}$ & $\begin{array}{l}\text { Direction } \\
\quad(a z)\end{array}$ & $\begin{array}{l}\text { Maximum } \\
\text { ampl i tude } \\
(\text { g) }\end{array}$ & $\begin{array}{l}\text { Duration } \\
\text { (s) }\end{array}$ \\
\hline \multirow{14}{*}{$\begin{array}{l}24 \text { November } 1987 \\
1315: 56.4 \text { G.m.t. } \\
\text { Southern Calif. } \\
33.010 \mathrm{~N}, 115.840 \mathrm{~W} \\
\text { Magnitude } 6.6 \mathrm{Ms--} \\
\text { Cont inued }\end{array}$} & $\begin{array}{l}\text { E1 Centro Array } 7 \\
\text { Imp. VaI ley College } \\
\text { (USGS) }\end{array}$ & $\begin{array}{r}32.829 \mathrm{~N} \\
115.504 \mathrm{~W}\end{array}$ & $16: 04.7$ & 5.6 & $\begin{array}{l}230 \\
\text { Up } \\
140\end{array}$ & $\begin{array}{l}.20 \\
.18 \\
.26\end{array}$ & $\begin{array}{l}3.6 \\
0.5 \\
2.9\end{array}$ \\
\hline & $\begin{array}{l}\text { El Centro Diff. Array } \\
\text { Dogwood Road } \\
\text { USGS) }\end{array}$ & $\begin{array}{r}32.796 \mathrm{~N} \\
115.535 \mathrm{~W}\end{array}$ & $16: 07.2$ & 3.5 & $\begin{array}{l}360 \\
\text { Up } \\
270\end{array}$ & $\begin{array}{l}.29 \\
.14 \\
.23\end{array}$ & $\begin{array}{r}13.4 \\
3.4 \\
11.7\end{array}$ \\
\hline & $\begin{array}{l}\text { E } 1 \text { Centro Array } 6 \\
\text { Huston Road } \\
\text { (USGS) }\end{array}$ & $\begin{array}{r}32.839 \mathrm{~N} \\
115.487 \mathrm{~W}\end{array}$ & $16: 04.9$ & 4.2 & $\begin{array}{l}230 \\
\text { Up } \\
140\end{array}$ & $\begin{array}{l}.16 \\
.14 \\
.19\end{array}$ & $\begin{array}{l}2.4 \\
2.0 \\
0.7\end{array}$ \\
\hline & $\begin{array}{l}\text { El Centro Array } 5 \\
\text { James Road } \\
\text { (USGS) }\end{array}$ & $\begin{array}{r}32.855 \mathrm{~N} \\
115.466 \mathrm{~W}\end{array}$ & $16: 05.5$ & 5.9 & $\begin{array}{l}230 \\
\text { Up } \\
140\end{array}$ & $\begin{array}{l}.19 \\
.11 \\
.20\end{array}$ & $\begin{array}{l}3.9 \\
1-\text { peak } \\
2.6\end{array}$ \\
\hline & $\begin{array}{l}\text { E1 Centro } \\
\text { Meadows Union School } \\
\text { (USGS) }\end{array}$ & $\begin{array}{r}32.800 \mathrm{~N} \\
115.473 \mathrm{~W}\end{array}$ & $16: 05.4$ & 6.2 & $\begin{array}{l}230 \\
\text { Up } \\
140\end{array}$ & $\begin{array}{l}.27 \\
.12 \\
.26\end{array}$ & $\begin{array}{c}8.7 \\
1-\text { peak } \\
9.3\end{array}$ \\
\hline & $\begin{array}{l}\text { E1 Centro Array } 4 \\
\text { Anderson Road } \\
\text { (USGS) }\end{array}$ & $\begin{array}{r}32.864 \mathrm{~N} \\
115.432 \mathrm{~W}\end{array}$ & $16: 06.6$ & 5.2 & $\begin{array}{l}230 \\
\text { Up } \\
140\end{array}$ & $\begin{array}{l}.10 \\
.12 \\
.11\end{array}$ & $\begin{array}{c}1 \text {-peak } \\
1 \text {-peak } \\
0.8\end{array}$ \\
\hline & $\begin{array}{l}\text { Borrego Springs } \\
\text { Scripps Clinic } \\
\text { (USGS) }\end{array}$ & $\begin{array}{r}33.210 \mathrm{~N} \\
116.330 \mathrm{~W}\end{array}$ & $16: 08.1$ & 6.9 & $\begin{array}{l}315 \\
\text { Up } \\
225\end{array}$ & $\begin{array}{l}.06 \\
.05 \\
.06\end{array}$ & $\begin{array}{l}--- \\
-- \\
---\end{array}$ \\
\hline & $\begin{array}{l}\text { El Centro Array } 3 \\
\text { Pine Un ion School } \\
\text { (USGS) }\end{array}$ & $\begin{array}{r}32.894 \mathrm{~N} \\
115.380 \mathrm{~W}\end{array}$ & $16: 06.5$ & 7.1 & $\begin{array}{l}230 \\
\text { Up } \\
140\end{array}$ & $\begin{array}{l}.11 \\
.08 \\
.11\end{array}$ & $\begin{array}{c}1.8 \\
1 \text { peak }\end{array}$ \\
\hline & $\begin{array}{l}\text { E1 Centro Array } 2 \\
\text { Keystone Road } \\
\text { (USGS) }\end{array}$ & $\begin{array}{r}32.916 \mathrm{~N} \\
115.366 \mathrm{~W}\end{array}$ & $16: 06.3$ & 6.9 & $\begin{array}{l}230 \\
\text { Up } \\
140\end{array}$ & $\begin{array}{l}.12 \\
.05 \\
.10\end{array}$ & $\begin{array}{c}0.9 \\
--- \\
1-\text { peak }\end{array}$ \\
\hline & $\begin{array}{l}\text { El Centro Array } 1 \\
\text { Borchard Ranches } \\
\text { (USGS) }\end{array}$ & $\begin{array}{r}32.960 \mathrm{~N} \\
115.319 \mathrm{~W}\end{array}$ & $16: 13.5$ & 7.1 & $\begin{array}{l}230 \\
\text { Up } \\
140\end{array}$ & $\begin{array}{l}.09 \\
.06 \\
.09\end{array}$ & $\begin{array}{l}--- \\
---\end{array}$ \\
\hline & $\begin{array}{l}\text { Calexico } \\
\text { Fire Station } \\
\text { (USGS) }\end{array}$ & $\begin{array}{r}32.669 \mathrm{~N} \\
115.492 \mathrm{~W}\end{array}$ & $16: 06.5$ & 7.2 & $\begin{array}{l}315 \\
\text { Up } \\
225\end{array}$ & $\begin{array}{l}.21 \\
.10 \\
.21\end{array}$ & $\begin{array}{r}10.5 \\
6.0 \\
11.7\end{array}$ \\
\hline & $\begin{array}{l}\text { Coachella Canal } \\
\text { Station } 3 \\
\text { (USGS) }\end{array}$ & $\begin{array}{r}33.51 \mathrm{~N} \\
115.77 \mathrm{~W}\end{array}$ & (3) & 6.1 & $\begin{array}{l}135 \\
\text { Up } \\
045\end{array}$ & $\begin{array}{l}.09 \\
.05 \\
.08\end{array}$ & $\begin{array}{l}--- \\
---\end{array}$ \\
\hline & $\begin{array}{l}\text { Bonds Corner } \\
\text { Highway } 98 \text { at } 115 \\
\text { (USGS) (SMA) }\end{array}$ & $\begin{array}{r}32.693 \mathrm{~N} \\
115.338 \mathrm{~W}\end{array}$ & $16: 08.5$ & 8.2 & $\begin{array}{l}230 \\
\text { Up } \\
140\end{array}$ & $\begin{array}{l}.28 \\
.09 \\
.28\end{array}$ & $\begin{array}{l}9.9 \\
9.4\end{array}$ \\
\hline & $\begin{array}{l}\text { Bonds Corner } \\
\text { Highway } 98 \text { at } 115 \\
\text { (USGS) (RFT) }\end{array}$ & $\begin{array}{r}32.693 \mathrm{~N} \\
115.338 \mathrm{~W}\end{array}$ & $16: 08.5$ & 8.2 & $\begin{array}{l}230 \\
\text { Up } \\
140\end{array}$ & $\begin{array}{l}.27 \\
.08 \\
.29\end{array}$ & $\begin{array}{l}9.8 \\
9.5\end{array}$ \\
\hline
\end{tabular}


Table 1. Catalogue of National Cooperative Strong-Motion Network accelerograph records recovered during 1987-Continued

\begin{tabular}{|c|c|c|c|c|c|c|c|}
\hline Earthquake & $\begin{array}{l}\text { Station name } \\
\text { (owner) }\end{array}$ & $\begin{array}{l}\text { Station } \\
\text { location } \\
\left({ }^{\circ}\right)\end{array}$ & $\begin{array}{l}\text { Trigger } \\
\text { time }\end{array}$ & $\begin{array}{l}\text { S-minus } \\
\text { trigger } \\
(\mathrm{s})\end{array}$ & $\begin{array}{l}\text { Direction } \\
\quad(a z)\end{array}$ & $\begin{array}{l}\text { Maximum } \\
\text { amplitude } \\
\text { (g) }\end{array}$ & $\begin{array}{l}\text { Duration } \\
\text { (s) }\end{array}$ \\
\hline \multirow{13}{*}{$\begin{array}{l}24 \text { November } 1987 \\
1315: 56.4 \text { G.m.t. } \\
\text { Southern Calif. } \\
33.010 \mathrm{~N}, 115.840 \mathrm{~W} \\
\text { Magnitude } 6.6 \mathrm{Ms}-- \\
\text { Cont inued }\end{array}$} & $\begin{array}{l}\text { Coachel la Canal } \\
\text { Station } 2 \\
\text { (USGS) }\end{array}$ & $\begin{array}{r}33.56 \mathrm{~N} \\
115.95 \mathrm{~W}\end{array}$ & (3) & (2) & $\begin{array}{l}135 \\
\text { Up } \\
045\end{array}$ & $\begin{array}{l}.04 \\
.03 \\
.04\end{array}$ & $\begin{array}{l}--- \\
---\end{array}$ \\
\hline & $\begin{array}{l}\text { Rancho de Anza } \\
\text { Anza-Borrego Park } \\
\text { (USGS) }\end{array}$ & $\begin{array}{r}33.35 \mathrm{~N} \\
116.40 \mathrm{~W}\end{array}$ & $16: 19.1$ & (2) & $\begin{array}{l}135 \\
\text { Up } \\
045\end{array}$ & $\begin{array}{l}.06 \\
.03 \\
.06\end{array}$ & $\begin{array}{l}--- \\
--- \\
---\end{array}$ \\
\hline & $\begin{array}{l}\text { Coachella Canal } \\
\text { Station } 1 \\
\text { (USGS) }\end{array}$ & $\begin{array}{r}33.64 \mathrm{~N} \\
116.08 \mathrm{~W}\end{array}$ & (3) & 9.6 & $\begin{array}{l}135 \\
\text { Up } \\
045\end{array}$ & $\begin{array}{l}.08 \\
.05 \\
.09\end{array}$ & $\begin{array}{l}--- \\
---\end{array}$ \\
\hline & $\begin{array}{l}\text { Terwilliger Valley } \\
\text { Anza Array } \\
\text { (USGS) }\end{array}$ & $\begin{array}{r}33.48 \mathrm{~N} \\
116.59 \mathrm{~W}\end{array}$ & (3) & (2) & $\begin{array}{l}135 \\
\text { Up } \\
045\end{array}$ & $\begin{array}{l}.02 \\
.01 \\
.02\end{array}$ & $\begin{array}{l}--- \\
---\end{array}$ \\
\hline & $\begin{array}{l}\text { Chihuahua } \\
\text { Anza Array } \\
\text { (USGS) }\end{array}$ & $\begin{array}{r}33.38 \mathrm{~N} \\
116.68 \mathrm{~W}\end{array}$ & $16: 26.1$ & (2) & $\begin{array}{l}360 \\
U p \\
270\end{array}$ & $\begin{array}{l}.02 \\
.01 \\
.03\end{array}$ & $\begin{array}{l}--- \\
---\end{array}$ \\
\hline & $\begin{array}{l}\text { Pinyon Flat observ. } \\
\text { Anza Array } \\
\text { (USGS) }\end{array}$ & $\begin{array}{r}33.61 \mathrm{~N} \\
116.46 \mathrm{~W}\end{array}$ & $16: 24.95$ & (2) & $\begin{array}{l}135 \\
\text { Up } \\
045\end{array}$ & $\begin{array}{l}.01 \\
.01 \\
.01\end{array}$ & $\begin{array}{l}--- \\
--- \\
---\end{array}$ \\
\hline & $\begin{array}{l}\text { Ind io } \\
\text { So. Cal if. Gas Co. } \\
\text { (USGS) }\end{array}$ & $\begin{array}{r}33.747 \mathrm{~N} \\
116.214 \mathrm{~W}\end{array}$ & $16: 16.0$ & 8.4 & $\begin{array}{l}315 \\
\text { Up } \\
225\end{array}$ & $\begin{array}{l}.03 \\
.03 \\
.04\end{array}$ & $\begin{array}{l}--- \\
--- \\
---\end{array}$ \\
\hline & $\begin{array}{l}\text { Tule Canyon } \\
\text { Anza Array } \\
\text { (USGS) }\end{array}$ & $\begin{array}{r}33.47 \mathrm{~N} \\
116.64 \mathrm{~W}\end{array}$ & (3) & (2) & $\begin{array}{l}360 \\
\text { Up } \\
270\end{array}$ & $\begin{array}{l}.03 \\
.02 \\
.02\end{array}$ & $\begin{array}{l}--- \\
--- \\
--\end{array}$ \\
\hline & $\begin{array}{l}\text { Cahuilla Valley } \\
\text { Anza Array } \\
\text { (USGS) }\end{array}$ & $\begin{array}{r}33.512 \mathrm{~N} \\
116.798 \mathrm{~W}\end{array}$ & $16: 30.8$ & (2) & $\begin{array}{l}360 \\
U p \\
270\end{array}$ & $\begin{array}{l}.05 \\
.01 \\
.04\end{array}$ & $\begin{array}{l}--- \\
--- \\
---\end{array}$ \\
\hline & $\begin{array}{l}\text { Garner Valley } \\
\text { Anza Array } \\
\text { (USGS) }\end{array}$ & $\begin{array}{r}33.615 \mathrm{~N} \\
116.626 \mathrm{~W}\end{array}$ & $16: 27.5$ & (2) & $\begin{array}{l}360 \\
\text { Up } \\
270\end{array}$ & $\begin{array}{l}.02 \\
.01 \\
.02\end{array}$ & $\begin{array}{l}--- \\
--- \\
--\end{array}$ \\
\hline & $\begin{array}{l}\text { Thousand Palms } \\
\text { Post office } \\
\text { (USGS) }\end{array}$ & $\begin{array}{r}33.82 \mathrm{~N} \\
116.40 \mathrm{~W}\end{array}$ & (3) & (2) & $\begin{array}{l}135 \\
U p \\
045\end{array}$ & $\begin{array}{l}.02^{*} \\
.02^{*} \\
.03^{*}\end{array}$ & --- \\
\hline & Note: Event/recor & correlation & questiona & able. & & & \\
\hline & $\begin{array}{l}\text { N. Palm Springs } \\
\text { Post 0ffice } \\
\text { (USGS) }\end{array}$ & $\begin{array}{r}33.924 \mathrm{~N} \\
116.543 \mathrm{~W}\end{array}$ & $16: 20.6$ & (2) & $\begin{array}{l}300 \\
\text { Up } \\
210\end{array}$ & $\begin{array}{l}.02 \\
.01 \\
.02\end{array}$ & $\begin{array}{l}--- \\
---\end{array}$ \\
\hline $\begin{array}{l}24 \text { November } 1987 \\
1318 \text { G.m.t. } \\
\text { Southern Calif. }\end{array}$ & $\begin{array}{l}\text { Salton Sea } \\
\text { Wildlife Refuge } \\
\text { (USGS) }\end{array}$ & $\begin{array}{r}33.18 \mathrm{~N} \\
115.62 \mathrm{~W}\end{array}$ & $18: 11.9$ & 1.0 & $\begin{array}{l}315 \\
\text { Up } \\
225\end{array}$ & $\begin{array}{l}.21 \\
.18 \\
.16\end{array}$ & $\begin{array}{l}0.5 \\
0.7 \\
0.3\end{array}$ \\
\hline
\end{tabular}


Table 1. Catalogue of National Cooperative Strong-Motion Network accelerograph records recovered during 1987-Continued

\begin{tabular}{|c|c|c|c|c|c|c|c|}
\hline Earthquake & $\begin{array}{l}\text { Station name } \\
\text { (owner) }\end{array}$ & $\begin{array}{l}\text { Station } \\
\text { location } \\
\left({ }^{\circ}\right)\end{array}$ & $\begin{array}{l}\text { Trigger } \\
\text { time }\end{array}$ & $\begin{array}{l}\text { S-minus } \\
\text { trigger } \\
(\mathrm{s})\end{array}$ & $\begin{array}{l}\text { Direction } \\
\quad(a z)\end{array}$ & $\begin{array}{l}\text { Maximum D } \\
\text { amplitude } \\
\text { (g) }\end{array}$ & $\begin{array}{l}\text { Duration } \\
\text { (s) }\end{array}$ \\
\hline $\begin{array}{l}24 \text { November } 1987 \\
1324 \text { G.m.t. } \\
\text { Southern Calif. } \\
\text { Epicenter and } \\
\text { magnitude unknown }\end{array}$ & $\begin{array}{l}\text { Superstition Mountain } \\
\text { Camera Site } 8 \\
\text { (USGS) }\end{array}$ & $\begin{array}{r}32.955 \mathrm{~N} \\
115.823 \mathrm{~W}\end{array}$ & $24: 41.7$ & (2) & $\begin{array}{l}135 \\
\text { Up } \\
045\end{array}$ & $\begin{array}{l}.11 \\
.05 \\
.05\end{array}$ & 1 peak \\
\hline $\begin{array}{l}24 \text { November } 1987 \\
1330 \text { G.m.t. } \\
\text { Southern Ca1 if. } \\
\text { Epicenter and } \\
\text { magnitude unknown }\end{array}$ & $\begin{array}{l}\text { Superstition Mounta in } \\
\text { Camera Site } 8 \\
\text { (USGS) }\end{array}$ & $\begin{array}{r}32.955 \mathrm{~N} \\
115.823 \mathrm{~W}\end{array}$ & $30: 04.3$ & (2) & & (1) & \\
\hline \multirow{2}{*}{$\begin{array}{l}24 \text { November } 198.7 \\
1332: 59.8 \text { G.m.t. } \\
\text { Southern Ca7 if. } \\
32.990 \mathrm{~N}, 115.870 \mathrm{~W} \\
\text { Magnitude } 4.2 \mathrm{ML}\end{array}$} & $\begin{array}{l}\text { Superstition Mountain } \\
\text { Camera Site } 8 \\
\text { (USGS) }\end{array}$ & $\begin{array}{r}32.955 \mathrm{~N} \\
115.823 \mathrm{~W}\end{array}$ & $33: 07.4$ & (2) & $\begin{array}{l}135 \\
\text { Up } \\
045\end{array}$ & $\begin{array}{l}.06 \\
.03 \\
.06\end{array}$ & $\begin{array}{l}--- \\
--- \\
---\end{array}$ \\
\hline & \multicolumn{4}{|c|}{ Two additional records ${ }^{1}$ recovered at Superstition } & Mounta in & Camera Site & 8 \\
\hline \multirow{9}{*}{$\begin{array}{l}24 \text { November } 1987 \\
1334: 39.9 \text { G.m.t. } \\
\text { Southern Calif. } \\
32.940 \mathrm{~N}, 115.760 \mathrm{~W} \\
\text { Magnitude } 4.8 \mathrm{ML}\end{array}$} & $\begin{array}{l}\text { Superstition Mountain } \\
\text { Camera Site } 8 \\
\text { (USGS) }\end{array}$ & $\begin{array}{r}32.955 \mathrm{~N} \\
115.823 \mathrm{~W}\end{array}$ & $34: 41.8$ & 1.4 & $\begin{array}{l}135 \\
\text { Up } \\
045\end{array}$ & $\begin{array}{l}.12 \\
.12 \\
.11\end{array}$ & $\begin{array}{l}0.5 \\
1 \text { peak } \\
1 \text { peak }\end{array}$ \\
\hline & $\begin{array}{l}\text { El Centro } \\
\text { Parachute Test Site } \\
\text { (USGS) }\end{array}$ & $\begin{array}{r}32.93 \mathrm{~N} \\
115.70 \mathrm{~W}\end{array}$ & $34: 42.4$ & 1.9 & $\begin{array}{l}315 \\
\text { Up } \\
225\end{array}$ & $\begin{array}{l}.16 \\
.08 \\
.09\end{array}$ & $\begin{array}{ll}0.3 \\
---\end{array}$ \\
\hline & $\begin{array}{l}\text { Plaster City } \\
\text { Storehouse } \\
\text { (USGS) }\end{array}$ & $\begin{array}{r}32.79 \mathrm{~N} \\
115.86 \mathrm{~W}\end{array}$ & $34: 43.7$ & 2.6 & & (1) & \\
\hline & $\begin{array}{l}\text { El Centro Array } 8 \\
\text { Cruickshank Road } \\
\text { (USGS) }\end{array}$ & $\begin{array}{r}32.811 \mathrm{~N} \\
115.532 \mathrm{~W}\end{array}$ & $34: 45.5$ & 4.4 & & (1) & \\
\hline & $\begin{array}{l}\text { El Centro Array } 7 \\
\text { Imp. Valley College } \\
\text { (USGS) }\end{array}$ & $\begin{array}{r}32.829 \mathrm{~N} \\
115.504 \mathrm{~W}\end{array}$ & $34: 45.7$ & 4.5 & & (1) & \\
\hline & $\begin{array}{l}\text { El Centro Array } 11 \\
\text { MaCabe School } \\
\text { (USGS) }\end{array}$ & $\begin{array}{r}32.752 \mathrm{~N} \\
115.594 \mathrm{~W}\end{array}$ & $34: 45.9$ & 4.3 & & (1) & \\
\hline & $\begin{array}{l}\text { Salton Sea } \\
\text { Wildlife Refuge } \\
\text { (USGS) }\end{array}$ & $\begin{array}{r}33.18 \mathrm{~N} \\
115.62 \mathrm{~W}\end{array}$ & $34: 46.0$ & 4.5 & & (1) & \\
\hline & $\begin{array}{l}\text { E1 Centro Array } 6 \\
\text { Huston Road } \\
\text { (USGS) }\end{array}$ & $\begin{array}{r}32.839 \mathrm{~N} \\
115.487 \mathrm{~W}\end{array}$ & $34: 46.4$ & 4.3 & & (1) & \\
\hline & $\begin{array}{l}\text { E1 Centro Array } 4 \\
\text { Anderson Road } \\
\text { (USGS) }\end{array}$ & $\begin{array}{r}32.86 \mathrm{~N} \\
115.32 \mathrm{~W}\end{array}$ & $34: 46.7$ & 5.3 & & (1) & \\
\hline
\end{tabular}


Table 1. Catalogue of National Cooperative Strong-Motion Network accelerograph records recovered during 1987-Continued

\begin{tabular}{|c|c|c|c|c|c|c|c|}
\hline Earthquake & $\begin{array}{l}\text { Station name } \\
\text { (owner) }\end{array}$ & $\begin{array}{l}\text { Station } \\
\text { location } \\
\left({ }^{\circ}\right)\end{array}$ & $\begin{array}{l}\text { Trigger } \\
\text { time }\end{array}$ & $\begin{array}{l}\text { S-minus } \\
\text { trigger } \\
(\mathrm{s})\end{array}$ & $\begin{array}{l}\text { Direction } \\
\quad(a z)\end{array}$ & $\begin{array}{l}\text { Maximun } \\
\text { ampliture } \\
\text { (g) }\end{array}$ & $\begin{array}{l}\text { Duration } \\
\text { (s) }\end{array}$ \\
\hline $\begin{array}{l}24 \text { November } 1987 \\
1334: 39.9 \text { G.m.t. } \\
\text { Southern Cal if. } \\
32.940 \mathrm{~N}, 115.760 \mathrm{~W} \\
\text { Magn itude } 4.8 \mathrm{ML}-- \\
\text { Cont inued }\end{array}$ & $\begin{array}{l}\text { Imperial Wildlife } \\
\text { Liquefaction Array } \\
\text { (USGS) }\end{array}$ & $\begin{array}{r}33.097 \mathrm{~N} \\
115.530 \mathrm{~W}\end{array}$ & (3) & 5.4 & & (1) & \\
\hline \multirow{2}{*}{$\begin{array}{l}24 \text { November 1987- } \\
25 \text { November } 1987 \\
\text { Southern Cal if. } \\
\text { Epicenter and } \\
\text { maynitude unknown }\end{array}$} & $\begin{array}{l}\text { Calipatria } \\
\text { Fire Station } \\
\text { (USGS) }\end{array}$ & $\begin{array}{r}33.13 \mathrm{~N} \\
115.52 \mathrm{~W}\end{array}$ & (3) & 5.4 & $\begin{array}{l}315 \\
\text { Up } \\
225\end{array}$ & $\begin{array}{l}.09 \\
.02 \\
.03\end{array}$ & $\begin{array}{l}-- \\
--- \\
---\end{array}$ \\
\hline & $\begin{array}{l}\text { Brawley } \\
\text { A irport Hangar } \\
\text { (USGS) }\end{array}$ & $\begin{array}{l}32.988 \mathrm{~N}, \\
115.509 \mathrm{~W}\end{array}$ & (3) & 1.1 & $\begin{array}{l}315 \\
U p \\
225\end{array}$ & $\begin{array}{l}.08 \\
.02 \\
.09\end{array}$ & $\begin{array}{l}-- \\
--- \\
--\end{array}$ \\
\hline \multirow{2}{*}{$\begin{array}{l}24-25 \text { Nov. } 1987 \\
1316-0230 \text { G.m.t. } \\
\text { Southern Calif. } \\
\text { Epicenters and } \\
\text { magnitudes unknown }\end{array}$} & $\begin{array}{l}\text { Salton Sea, KNB } \\
\text { Kornbloom (Temp.) } \\
\text { (CIT) }\end{array}$ & $\begin{array}{r}33.125 \mathrm{~N} \\
115.665 \mathrm{~W}\end{array}$ & (3) & 1.7 & $\begin{array}{l}180 \\
\text { Up } \\
090\end{array}$ & $\begin{array}{l}.07 \\
.02 \\
.06\end{array}$ & $\begin{array}{l}-- \\
--- \\
--\end{array}$ \\
\hline & Note: One addit & al record ${ }^{1}$ & recovered & at Salto & on Sea, KNB. & & \\
\hline \multirow{7}{*}{$\begin{array}{l}24-25 \text { Nov. } 1987 \\
1316-0346 \text { G.m.t. } \\
\text { Southern Cal if. } \\
\text { Epicenters and } \\
\text { magnitudes unknown }\end{array}$} & $\begin{array}{l}\text { Salton Sea, POE } \\
\text { Poe Road (Temp.) } \\
\text { (CIT) }\end{array}$ & $\begin{array}{r}33.097 \mathrm{~N} \\
115.751 \mathrm{~W}\end{array}$ & (3) & 0.7 & $\begin{array}{l}180 \\
\text { Up } \\
090\end{array}$ & $\begin{array}{l}.07 \\
.05 \\
.05\end{array}$ & $\begin{array}{l}--- \\
---\end{array}$ \\
\hline & & & (3) & 3.0 & $\begin{array}{l}180 \\
\text { Up } \\
090\end{array}$ & $\begin{array}{l}.06 \\
.03 \\
.08\end{array}$ & $\begin{array}{l}--- \\
---\end{array}$ \\
\hline & & & (3) & 2.1 & $\begin{array}{l}180 \\
\text { Up } \\
090\end{array}$ & $\begin{array}{l}.05 \\
.02 \\
.04\end{array}$ & $\begin{array}{l}--- \\
--- \\
---\end{array}$ \\
\hline & & & (3) & 3.1 & $\begin{array}{l}180 \\
\text { Up } \\
090\end{array}$ & $\begin{array}{l}.05 \\
.06 \\
.05\end{array}$ & $\begin{array}{l}--- \\
--- \\
---\end{array}$ \\
\hline & & & (3) & (2) & $\begin{array}{l}180 \\
\text { Up } \\
090\end{array}$ & $\begin{array}{l}.06 \\
.01 \\
.06\end{array}$ & $\begin{array}{l}--- \\
--- \\
--\end{array}$ \\
\hline & & & (3) & 1.8 & $\begin{array}{l}180 \\
\text { Up } \\
090\end{array}$ & $\begin{array}{l}.04 \\
.03 \\
.09\end{array}$ & $\begin{array}{l}--- \\
--- \\
---\end{array}$ \\
\hline & & & (3) & 1.5 & $\begin{array}{l}180 \\
\text { Up } \\
090\end{array}$ & $\begin{array}{l}.35 \\
.17 \\
.14\end{array}$ & $\begin{array}{l}0.6 \\
0.3 \\
0.3\end{array}$ \\
\hline
\end{tabular}

Note: Five additional records ${ }^{l}$ recovered at Salton Sea, POE. 
Table 1. Catalogue of National Cooperative Strong-Motion Network accelerograph records recovered during 1987-Continued

\begin{tabular}{|c|c|c|c|c|c|c|c|}
\hline Earthquake & $\begin{array}{l}\text { Station name } \\
\text { (owner) }\end{array}$ & $\begin{array}{l}\text { Station } \\
\text { location } \\
\left({ }^{\circ}\right)\end{array}$ & $\begin{array}{l}\text { Trigger } \\
\text { time }\end{array}$ & $\begin{array}{l}\text { S-minus } \\
\text { trigger } \\
(\mathrm{s})\end{array}$ & $\begin{array}{l}\text { Direction } \\
\quad(\mathrm{az})\end{array}$ & $\begin{array}{l}\text { Maximum } \\
\text { ampli tude } \\
\text { (g) }\end{array}$ & $\begin{array}{l}\text { Duration } \\
\text { (s) }\end{array}$ \\
\hline $\begin{array}{l}25 \text { November } 1987 \\
0346: 51.7 \mathrm{G} . \mathrm{m} . \mathrm{t} \text {. } \\
\text { Southern Calif. } \\
33.010 \mathrm{~N}, 115.850 \mathrm{~W} \\
\text { Magnitude } 3.4 \mathrm{ML}\end{array}$ & $\begin{array}{l}\text { Superstition Hills } \\
\text { East (Temp.) } \\
\text { (USGS) }\end{array}$ & $\begin{array}{r}33.005 \mathrm{~N} \\
115.729 \mathrm{~W}\end{array}$ & $46: 54.5^{4}$ & 2.8 & & (1) & \\
\hline $\begin{array}{l}25 \text { November } 1987 \\
0430: 17.8 \text { G.m.t. } \\
\text { Southern Cal if. } \\
32.980 \mathrm{~N}, 115.820 \mathrm{~W} \\
\text { Magnitude } 3.4 \mathrm{ML}\end{array}$ & $\begin{array}{l}\text { Superstition Hills } \\
\text { East (Temp.) } \\
\text { (USGS) }\end{array}$ & $\begin{array}{r}33.005 \mathrm{~N} \\
115.729 \mathrm{~W}\end{array}$ & $30: 20.0^{4}$ & 2.4 & $\begin{array}{l}360 \\
\text { up } \\
270\end{array}$ & $\begin{array}{l}.04 \\
.03 \\
.05\end{array}$ & $\overline{--}$ \\
\hline $\begin{array}{l}25 \text { November } 1987 \\
1354: 09.9 \text { G.m.t. } \\
\text { Southern Calif. } \\
32.980 \mathrm{~N}, 115.820 \mathrm{~W} \\
\text { Magnitude } 4.2 \mathrm{ML}\end{array}$ & $\begin{array}{l}\text { Superstition Hills } \\
\text { East (Temp.) } \\
\text { (USGS) }\end{array}$ & $\begin{array}{r}33.005 \mathrm{~N} \\
115.729 \mathrm{~W}\end{array}$ & $54: 12.1^{4}$ & 2.4 & $\begin{array}{l}360 \\
\text { Up } \\
270\end{array}$ & $\begin{array}{l}.09 \\
.11 \\
.14\end{array}$ & $\begin{array}{c}1 \text { peak } \\
0.1\end{array}$ \\
\hline $\begin{array}{l}25 \text { November } 1987 \\
1501: 37.9 \text { G.m.t. } \\
\text { Southern Calif. } \\
33.030 \mathrm{~N}, 115.780 \mathrm{~W} \\
\text { Magnitude } 3.2 \mathrm{ML}\end{array}$ & $\begin{array}{l}\text { Superstition Hills } \\
\text { East (Temp.) } \\
\text { (USGS) }\end{array}$ & $\begin{array}{r}33.005 \mathrm{~N} \\
115.729 \mathrm{~W}\end{array}$ & $01: 38.9^{4}$ & 2.1 & & (1) & \\
\hline $\begin{array}{l}26 \text { November } 1987 \\
0156: 27.5 \mathrm{G} . \mathrm{m} . \mathrm{t} \text {. } \\
\text { Southern Calif. } \\
32.990 \mathrm{~N}, 115.820 \mathrm{~W} \\
\text { Magnitude } 3.7 \mathrm{ML}\end{array}$ & $\begin{array}{l}\text { Superstition Hills } \\
\text { East (Temp.) } \\
\text { (USGS) }\end{array}$ & $\begin{array}{r}33.005 \mathrm{~N} \\
115.729 \mathrm{~W}\end{array}$ & $56: 30.0^{4}$ & 2.4 & & (1) & \\
\hline $\begin{array}{l}26 \text { November } 1987 \\
1739: 01.9 \text { G.m.t. } \\
\text { Southern Calif. } \\
33.030 \mathrm{~N}, 115.890 \mathrm{~W} \\
\text { Magnitude } 4.3 \mathrm{ML}\end{array}$ & $\begin{array}{l}\text { Superstition Hills } \\
\text { East (Temp.) } \\
\text { (USGS) }\end{array}$ & $\begin{array}{r}33.005 \mathrm{~N} \\
115.729 \mathrm{~W}\end{array}$ & $39: 05.6^{4}$ & 3.1 & & (1) & \\
\hline $\begin{array}{l}25 \text { November } 1987- \\
27 \text { November } 1987 \\
\text { Southern Calif. } \\
\text { Epicenters and }\end{array}$ & $\begin{array}{l}\text { Superstition Mountain } \\
\text { Camera Site } 8 \\
\text { (USGS) }\end{array}$ & $\begin{array}{r}32.955 \mathrm{~N} \\
115.823 \mathrm{~W}\end{array}$ & (3) & 0.5 & $\begin{array}{l}135 \\
\text { Up } \\
045\end{array}$ & $\begin{array}{l}.07 \\
.04 \\
.04\end{array}$ & $\begin{array}{l}--- \\
--- \\
---\end{array}$ \\
\hline magnitudes unknown & & & & 0.7 & $\begin{array}{l}135 \\
\text { Up } \\
045\end{array}$ & $\begin{array}{l}.17 \\
.10 \\
.10\end{array}$ & $\begin{array}{l}0.7 \\
1 \text { peak } \\
1 \text { peak }\end{array}$ \\
\hline
\end{tabular}

27 November 1987 0010 G.m.t. Southern Calif. Epicenter and magnitude unknown
Note: Four additional records ${ }^{1}$ recovered at Superstition Mountain Camera Site 8
Superstition Hills

East (Temp.)

(USGS)

$$
33.005 \mathrm{~N}
$$

$115.729 \mathrm{~W}$
$10: 48 \cdot 6^{4}$

2.1
.06
.02
.04
$--$ 
Table 1. Catalogue of National Cooperative Strong-Motion Network accelerograph records recovered during 1987-Continued

\begin{tabular}{|c|c|c|c|c|c|c|c|}
\hline Earthquake & $\begin{array}{l}\text { Station name } \\
\text { (owner) }\end{array}$ & $\begin{array}{l}\text { Station } \\
\text { location } \\
\left({ }^{\circ}\right)\end{array}$ & $\begin{array}{l}\text { Trigger } \\
\text { time }\end{array}$ & $\begin{array}{c}\text { S-minus } \\
\text { trigger } \\
(\mathrm{s})\end{array}$ & $\begin{array}{l}\text { Direction } \\
\quad(a z)\end{array}$ & $\begin{array}{l}\text { Maximum } \\
\text { amp l itude } \\
(\mathrm{g})\end{array}$ & $\begin{array}{c}\text { Duration } \\
(\mathrm{s})\end{array}$ \\
\hline $\begin{array}{l}27 \text { November } 1987 \\
0110: 10.5 \mathrm{G} . \mathrm{m} . \mathrm{t} . \\
\text { Southern Cal if. } \\
33.000 \mathrm{~N}, 115.820 \mathrm{~W} \\
\text { Magnitude } 4.7 \mathrm{ML}\end{array}$ & $\begin{array}{l}\text { Superstition Hills } \\
\text { East (Temp.) } \\
\text { (USGS) }\end{array}$ & $\begin{array}{r}33.005 \mathrm{~N} \\
115.729 \mathrm{~W}\end{array}$ & $10: 12.5^{4}$ & 2.2 & $\begin{array}{l}360 \\
\text { Up } \\
270\end{array}$ & $\begin{array}{l}.09 \\
.07 \\
.08\end{array}$ & $\begin{array}{l}--- \\
---\end{array}$ \\
\hline $\begin{array}{l}27 \text { November } 1987 \\
0615 \text { G.m.t. } \\
\text { Southern Calif. } \\
\text { Epicenter and } \\
\text { magnitude unknown }\end{array}$ & $\begin{array}{l}\text { E1 Centro, Parachute } \\
\text { Test Base (Temp.) } \\
\text { (USGS) }\end{array}$ & $\begin{array}{r}33.041 \mathrm{~N} \\
116.068 \mathrm{~W}\end{array}$ & $15: 49.9^{4}$ & (2) & $\begin{array}{l}135 \\
\text { Up } \\
045\end{array}$ & $\begin{array}{l}.10 \\
.08 \\
.10\end{array}$ & $\begin{array}{l}1 \text { peak } \\
1 \text { peak }\end{array}$ \\
\hline \multirow{3}{*}{$\begin{array}{l}27 \text { November } 1987 \\
\text { 0922:57.6 G.m.t. } \\
\text { Southern Calif. } \\
33.000 \mathrm{~N}, 115.81 \text { ow } \\
\text { Magn itude 4.1 ML }\end{array}$} & $\begin{array}{l}\text { Superstition Mountain } \\
\text { Camera Site } 8 \\
\text { (USGS) }\end{array}$ & $\begin{array}{r}32.955 \mathrm{~N} \\
115.823 \mathrm{~W}\end{array}$ & $22: 59.1$ & 1.0 & $\begin{array}{l}135 \\
\text { Up } \\
045\end{array}$ & $\begin{array}{l}.07 \\
.04 \\
.04\end{array}$ & $\begin{array}{l}--- \\
---\end{array}$ \\
\hline & $\begin{array}{l}\text { Superstition Hills } \\
\text { East (Temp.) } \\
\text { (USGS) }\end{array}$ & $\begin{array}{r}33.005 \mathrm{~N} \\
115.729 \mathrm{~W}\end{array}$ & $22: 59.5^{4}$ & 2.0 & $\begin{array}{l}360 \\
\text { Up } \\
270\end{array}$ & $\begin{array}{l}.06 \\
.07 \\
.05\end{array}$ & $\begin{array}{l}--- \\
---\end{array}$ \\
\hline & $\begin{array}{l}\text { E1 Centro, Parachute } \\
\text { Test Base (Temp.) } \\
\text { (USGS) }\end{array}$ & $\begin{array}{r}33.041 \mathrm{~N} \\
116.068 \mathrm{~W}\end{array}$ & $23: 04.5^{4}$ & (2) & & (1) & \\
\hline \multirow{5}{*}{$\begin{array}{l}28 \text { November } 1987 \\
0039: 10.9 \text { G.m.t. } \\
\text { Southern Calif. } \\
\text { 32.980N, 115.810W } \\
\text { Magnitude } 4.2 \mathrm{ML}\end{array}$} & $\begin{array}{l}\text { Superstition Mountain } \\
\text { Camera Site } 8 \\
\text { (USGS) }\end{array}$ & $\begin{array}{r}32.955 \mathrm{~N} \\
115.823 \mathrm{~W}\end{array}$ & $39: 11.3$ & 0.8 & $\begin{array}{l}135 \\
\text { Up } \\
045\end{array}$ & $\begin{array}{l}.07 \\
.06 \\
.07\end{array}$ & $\begin{array}{l}--- \\
--- \\
---\end{array}$ \\
\hline & $\begin{array}{l}\text { Superstition Hills } \\
\text { East (Temp.) } \\
\text { (USGS) }\end{array}$ & $\begin{array}{r}33.005 \mathrm{~N} \\
115.729 \mathrm{~W}\end{array}$ & $39: 12.9^{4}$ & 2.2 & $\begin{array}{l}360 \\
\text { Up } \\
270\end{array}$ & $\begin{array}{l}.10 \\
.09 \\
.12\end{array}$ & $\begin{array}{l}0.2 \\
--- \\
0.1\end{array}$ \\
\hline & $\begin{array}{l}\text { Superstition Mountain } \\
\text { Base (Temp) } \\
\text { (USGS) }\end{array}$ & $\begin{array}{r}32.960 \mathrm{~N} \\
115.816 \mathrm{~W}\end{array}$ & $39: 12.9^{4}$ & (2) & $\begin{array}{l}025 \\
\text { Up } \\
295\end{array}$ & $\begin{array}{l}.04 \\
.03 \\
.11\end{array}$ & 1 peak \\
\hline & $\begin{array}{l}\text { E1 Centro } \\
\text { Parachute Test Site } \\
\text { (USGS) }\end{array}$ & $\begin{array}{r}32.929 \mathrm{~N} \\
115.700 \mathrm{~W}\end{array}$ & $39: 16.1$ & (2) & & (1) & \\
\hline & $\begin{array}{l}\text { El Centro, Parachute } \\
\text { Test Base (Temp.) } \\
\text { (USGS) }\end{array}$ & $\begin{array}{r}32.926 \mathrm{~N} \\
115.695 \mathrm{~W}\end{array}$ & $39: 16.2^{4}$ & (2) & & (1) & \\
\hline \multirow{3}{*}{$\begin{array}{l}30 \text { November } 1987 \\
1923: 19.5 \mathrm{G} . \mathrm{m} . \mathrm{t} . \\
\text { Gulf of Alaska } \\
58.679 \mathrm{~N}, 142.786 \mathrm{~W} \\
\text { Magnitude } 7.1 \mathrm{ML}\end{array}$} & $\begin{array}{l}\text { Bancas Point } \\
\text { (USGS) }\end{array}$ & $\begin{array}{r}59.953 \mathrm{~N} \\
139.635 \mathrm{~W}\end{array}$ & $24: 53.4^{4}$ & (2) & $\begin{array}{l}360 \\
\text { Up } \\
270\end{array}$ & $\begin{array}{l}.05 \\
.02 \\
.06\end{array}$ & $\begin{array}{l}--- \\
--- \\
---\end{array}$ \\
\hline & $\begin{array}{l}\text { Middleton Island } \\
\text { (USGS) }\end{array}$ & $\begin{array}{r}59.443 \mathrm{~N} \\
146.332 \mathrm{~W}\end{array}$ & $23: 30.0^{4}$ & (2) & & (1) & \\
\hline & $\begin{array}{l}\text { Mt. Hamilton } \\
\text { (USGS) }\end{array}$ & $\begin{array}{r}60.337 N \\
144.261 W\end{array}$ & $24: 32.8^{4}$ & (2) & & (1) & \\
\hline
\end{tabular}


Table 1. Catalogue of National Cooperative Strong-Motion Network accelerograph records recovered during 1987-Continued

\begin{tabular}{|c|c|c|c|c|c|c|c|}
\hline Earthquake & $\begin{array}{l}\text { Station name } \\
\text { (owner) }\end{array}$ & $\begin{array}{l}\text { Station } \\
\text { location } \\
\left({ }^{\circ}\right)\end{array}$ & $\begin{array}{l}\text { Trigger } \\
\text { time }\end{array}$ & $\begin{array}{c}\text { S-minus } \\
\text { trigger } \\
(\mathrm{s})\end{array}$ & $\begin{array}{l}\text { Direction } \\
\quad(a z)\end{array}$ & $\begin{array}{l}\text { Maximum } \\
\text { amplitude } \\
(\mathrm{g})\end{array}$ & $\begin{array}{l}\text { Duration } \\
\text { (s) }\end{array}$ \\
\hline \multirow{4}{*}{$\begin{array}{l}30 \text { November } 1987 \\
1923: 19.5 \text { G.m.t. } \\
\text { Guif of Alaska } \\
58.679 \mathrm{~N}, 142.786 \mathrm{~W} \\
\text { Magn itude } 7.1 \mathrm{ML-} \\
\text { Continued }\end{array}$} & $\begin{array}{l}\text { Guyot HiTls } \\
\text { (USGS) }\end{array}$ & $\begin{array}{r}60.146 \mathrm{~N} \\
141.471 \mathrm{~W}\end{array}$ & $24: 31.9^{4}$ & (2) & $\begin{array}{l}360 \\
\text { Up } \\
270\end{array}$ & $\begin{array}{l}.03 \\
.05 \\
.06\end{array}$ & $\begin{array}{l}--- \\
---\end{array}$ \\
\hline & $\begin{array}{l}\text { Kayak Island } \\
\text { Ridge } \\
\text { USGS) }\end{array}$ & $\begin{array}{r}59.927 \mathrm{~N} \\
144.500 \mathrm{~W}\end{array}$ & $23: 57.6^{4}$ & (2) & & $(1)$ & \\
\hline & $\begin{array}{l}\text { Sunshine Point } \\
\text { (USGS) }\end{array}$ & $\begin{array}{r}60.180 \mathrm{~N} \\
142.838 \mathrm{~W}\end{array}$ & $24: 43.6^{4}$ & (2) & & (1) & \\
\hline & $\begin{array}{l}\text { Yakutat } \\
\text { FAA VOR Building } \\
\text { (USGS) }\end{array}$ & $\begin{array}{r}59.51 \mathrm{~N} \\
139.65 \mathrm{~W}\end{array}$ & (3) & 8.1 & $\begin{array}{l}360 \\
\text { Up } \\
270\end{array}$ & $\begin{array}{l}.12 \\
.05 \\
.14\end{array}$ & $\begin{array}{l}10 \\
-10.3\end{array}$ \\
\hline $\begin{array}{l}30 \text { November } 1987 \\
1954 \text { G.m.t. } \\
\text { Southern Calif. } \\
\text { Epicenter and } \\
\text { magnitude unknown }\end{array}$ & $\begin{array}{l}\text { Superstition Hills } \\
\text { East (Temp.) } \\
\text { (USGS) }\end{array}$ & $\begin{array}{r}33.005 \mathrm{~N} \\
115.729 \mathrm{~W}\end{array}$ & $54: 50.6^{4}$ & 2.2 & & (1) & \\
\hline \multirow{4}{*}{$\begin{array}{l}2 \text { December } 1987 \\
0403: 05.6 \text { G.m.t. } \\
\text { Southern Calif. } \\
\text { 33.126N, 115.830W } \\
\text { Magnitude } 4.6 \mathrm{ML}\end{array}$} & $\begin{array}{l}\text { Superstition Mountain } \\
\text { Camera Site } 8 \\
\text { (USGS) }\end{array}$ & $\begin{array}{r}32.955 \mathrm{~N} \\
115.823 \mathrm{~W}\end{array}$ & $03: 07.3$ & 0.5 & $\begin{array}{l}135 \\
\text { Up } \\
045\end{array}$ & $\begin{array}{l}.08 \\
.05 \\
.07\end{array}$ & $\overline{--}$ \\
\hline & $\begin{array}{l}\text { Superstition Hills } \\
\text { East (Temp.) } \\
\text { (USGS) }\end{array}$ & $\begin{array}{r}33.005 \mathrm{~N} \\
115.729 \mathrm{~W}\end{array}$ & $03: 08.1^{4}$ & 2.3 & $\begin{array}{l}360 \mathrm{~N} \\
\text { Up } \\
270\end{array}$ & $\begin{array}{l}.07 \\
.06 \\
.08\end{array}$ & $\begin{array}{l}--- \\
---\end{array}$ \\
\hline & $\begin{array}{l}\text { Superstition Mountain } \\
\text { Base (Temp.) } \\
\text { (USGS) }\end{array}$ & $\begin{array}{r}32.960 \mathrm{~N} \\
115.816 \mathrm{~W}\end{array}$ & $03: 08.7^{4}$ & (2) & $\begin{array}{l}025 \\
\text { Up } \\
295\end{array}$ & $\begin{array}{l}.05 \\
.03 \\
.09\end{array}$ & $\begin{array}{ll}--- \\
---\end{array}$ \\
\hline & $\begin{array}{l}\text { El Centro, Parachute } \\
\text { Test Base (Temp.) } \\
\text { (USGS) }\end{array}$ & $\begin{array}{r}32.926 \mathrm{~N} \\
115.695 \mathrm{~W}\end{array}$ & $03: 14.1^{4}$ & (2) & & (I) & \\
\hline $\begin{array}{l}3 \text { December } 1987 \\
1345: 57.9 \mathrm{G.m.t} . \\
\text { Southern Calif. } \\
33.000 \mathrm{~N}, 115.790 \mathrm{~W} \\
\text { Magn itude } 3.1 \mathrm{ML}\end{array}$ & $\begin{array}{l}\text { Superstition Hills } \\
\text { East (Temp.) } \\
\text { (USGS) }\end{array}$ & $\begin{array}{r}33.005 \mathrm{~N} \\
115.729 \mathrm{~W}\end{array}$ & $45: 59.8^{4}$ & (2) & & (1) & \\
\hline \multirow{2}{*}{$\begin{array}{l}3 \text { December } 1987 \\
1904: 36.5 \text { G.m.t. } \\
\text { Southern Calif. } \\
33.010 \mathrm{~N}, 115.870 \mathrm{~W} \\
\text { Magn itude } 3.8 \mathrm{ML}\end{array}$} & $\begin{array}{l}\text { Superstition Mounta in } \\
\text { Base (Temp.) } \\
\text { (USGS) }\end{array}$ & $\begin{array}{r}32.960 \mathrm{~N} \\
115.816 \mathrm{~W}\end{array}$ & $04: 40.0^{4}$ & (2) & & (1) & \\
\hline & $\begin{array}{l}\text { Superstition Mountain } \\
\text { Camera Site } 8 \\
\text { (USGS) }\end{array}$ & $\begin{array}{r}32.955 \mathrm{~W} \\
115.823 \mathrm{~W}\end{array}$ & (3) & (2) & & (1) & \\
\hline
\end{tabular}


Table 1. Catalogue of National Cooperative Strong-Motion Network accelerograph records recovired during 1987-Continued

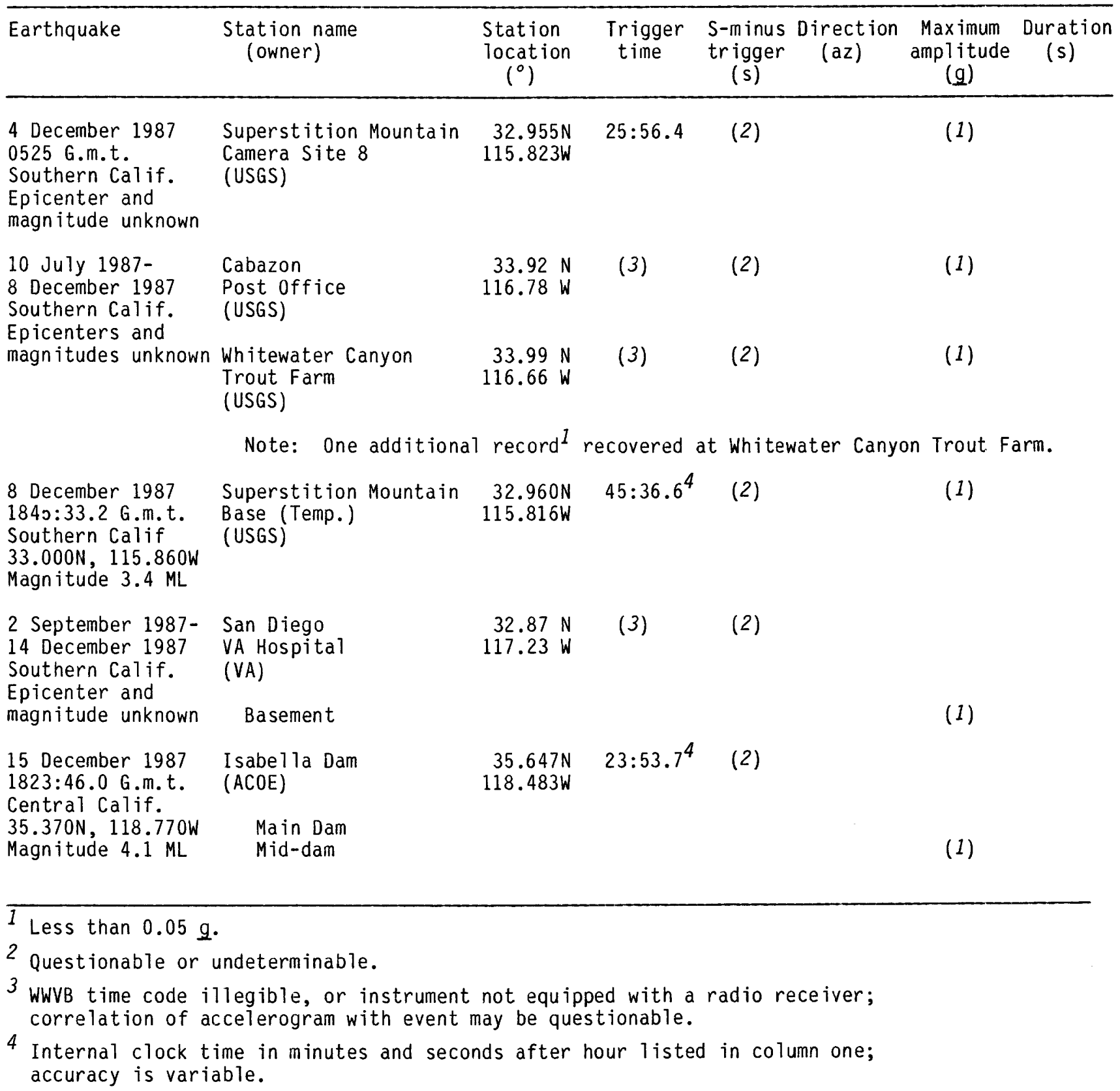





\section{SELECTED SERIES OF U.S. GEOLOGICAL SURVEY PUBLICATIONS}

\section{Perlodicals}

Earthquakes \& Volcanoes (issued bimonthly).

Preliminary Determination of Epicenters (issued monthly).

\section{Technical Books and Reports}

Professional Papers are mainly comprehensive scientific reports of wide and lasting interest and importance to professional scientists and engineers. Included are reports on the results of resource studies and of topographic, hydrologic, and geologic investigations. They also include collections of related papers addressing different aspects of a single scientific topic.

Bulletins contain significant data and interpretations that are of lasting scientific interest but are generally more limited in scope or geographic coverage than Professional Papers. They include the results of resource studies and of geologic and topographic investigations; as well as collections of short papers related to a specific topic.

Water-Supply Papers are comprehensive reports that present significant interpretive results of hydrologic investigations of wide interest to professional geologists, hydrologists, and engineers. The series covers investigations in all phases of hydrology, including hydrogeology, availability of water, quality of water, and use of water.

Circulars present administrative information or important scientific information of wide popular interest in a format designed for distribution at no cost to the public. Information is usually of short-term interest.

Water-Resources Investigations Reports are papers of an interpretive nature made available to the public outside the formal USGS publications series. Copies are reproduced on request unlike formal USGS publications, and they are also available for public inspection at depositories indicated in USGS catalogs.

Open-File Reports include unpublished manuscript reports, maps, and other material that are made available for public consultation at depositories. They are a nonpermanent form of publication that may be cited in other publications as sources of information.

\section{Maps}

Geologic Quadrangle Maps are multicolor geologic maps on topographic bases in 71/2-or 15 -minute quadrangle formats (scales mainly $1: 24,000$ or $1: 62,500$ ) showing bedrock, surficial, or engineering geology. Maps generally include brief texts; some maps include structure and columnar sections only.

Geophysical Investigations Maps are on topographic or planimetric bases at various scales; they show results of surveys using geophysical techniques, such as gravity, magnetic, seismic, or radioactivity, which reflect subsurface structures that are of economic or geologic significance. Many maps include correlations with the geology.

Miscellaneous Investigations Series Maps are on planimetric or topographic bases of regular and irregular areas at various scales; they present a wide variety of format and subject matter. The series also includes 7 1/2-minute quadrangle photogeologic maps on planimetric bases which show geology as interpreted from aerial photographs. Series also includes maps of Mars and the Moon.
Coal Investigations Maps are geologic maps on topographic or planimetric bases at various scales showing bedrock o- surficial geology, stratigraphy, and structural relations in certain coal-resource areas.

Oil and Gas Investigations Charts show stratigraphic information for certain oil and gas fields and other areas having petrcleum potential.

Miscellaneous Field Studies Maps are multicolo- or black-andwhite maps on topographic or planimetric bases on quadrangle or irregular areas at various scales. Pre-1971 maps show b?drock geology in relation to specific mining or mineral-deposit probl ms; post-1971 maps are primarily black-and-white maps on various subjects such as environmental studies or wilderness mineral investigations.

Hydrologic Investigations Atlases are multicolore or black-andwhite maps on topographic or planimetric bases presenting a wide range of geohydrologic data of both regular and irregular areas: principal scale is $1: 24,000$ and regional studies are at 1:250,000 scale or smaller.

\section{Catalogs}

Permanent catalogs, as well as some others, giving comprehensive listings of U.S. Geological Survey publications are available under the conditions indicated below from the U.S. Geologica' Survey, Books and Open-File Reports Section, Federal Center, Box 25425, Denver, CO 80225. (See latest Price and Availability List.)

"Publications of the Geological Survey, 1879- 1961" may be purchased by mail and over the counter in paperback boot form and as a set of microfiche.

"Publications of the Geological Survey, 1962- 1970" may be purchased by mail and over the counter in paperback boot form and as a set of microfiche.

"Publications of the U.S. Geological Survey, 1971- 1981" may be purchased by mail and over the counter in paperback book form (two volumes, publications listing and index) and as a set of microfiche.

Supplements for 1982, 1983, 1984, 1985, 1986, and for subsequent years since the last permanent catalog may be purchased by mail and over the counter in paperback book form.

State catalogs, "List of U.S. Geological Survey' Geologic and Water-Supply Reports and Maps For (State)," may be purchased by mail and over the counter in paperback booklet form only.

"Price and Availability List of U.S. Geological S rrey Publications," issued annually, is available free of charge in paperback booklet form only.

Selected copies of a monthly catalog "New Publications of the U.S. Geological Survey" available free of charge by mail or may be obtained over the counter in paperback booklet form only. Those wishing a free subscription to the monthly catalog "New Publicaticns of the U.S. Geological Survey" should write to the U.S. Geologiral Survey, 582 National Center, Reston, VA 22092.

Note.--Prices of Government publications listed in older catalogs, announcements, and publications may be incorrect. Therefore, the prices charged may differ from the prices in catalogs, ennouncements, and publications. 
